Espaços nos quais todo Fechado é um Conjunto de Pontos Fixos

\author{
Irene Castro Pereira
}

\author{
DISSERTAÇÃO APRESENTADA \\ $\mathrm{AO}$ \\ INSTITUTO DE MATEMÁTICA E ESTATÍSTICA \\ DA \\ UNIVERSIDADE DE SÃO PAULO \\ PARA \\ OBTENÇÃO DO GRAU DE MESTRE \\ EM \\ MATEMÁTICA
}

Área de Concentração: Topologia Geral e Conjuntística Orientador: Prof. Dr. Artur Hideyuki Tomita

Durante a elaboração deste trabalho o autor recebeu apoio financeiro do CAPES

-São Paulo. julho de 1998- 


\section{Espaços nos quais todo Fechado é um Conjunto de Pontos Fixos}

Este exemplar corresponde à redação

final da dissertaçào devidamente corrigida e defendida por Irene Castro

e aprovada pela comissão julgadora.

São Paulo, setembro de 1998.

Banca examinadora:

- Prof. Dr. Artur Hideyuki Tomita (Orientador) - IME-USP

- Prof. Dr. Ricardo Bianconi - IME-USP

- Prof. Dr. Newton Carneiro Affonso da Costa - FFLCH-USP 


\section{RESUMO}

Um espaço $X$ é dito ter a propriedade da invariância completa (CIP) se todo subconjunto fechado nào vazio de $X$ é um conjunto de pontos fixos. Neste trabalho vemos que a CIP não é preservada por auto-produto de variedades não métricas ou espaços zero-dimensionais. Vemos também condições suficientes para um produto infinito de espaços ter CIP. Mostramos que o produto não enumerável do intervalo unitário (o cubo de Tychonoff) não tem CIP e que o cubo de Hilbert e o cubo de Cantor tem a propriedade da invariância completa com respeito a homeomorfismos (CIPH).

\footnotetext{
ABSTRACT

A space $X$ is said to have the complete invariance property (CIP) if every nonempty closed subset of $X$ is the fixed point set of some self-mapping of $X$. In this work we see that CIP need not be preserved by self-products of non-metric manifolds or zero-dimensional spaces. We also see sufficient conditions for an infinite product of spaces to have CIP. We show that uncountable powers of the unit interval do not have CIP and that the Hilbert cube and the Cantor cube have the complete invariance property with respect to homeomorphisms (CIPH).
} 
A mis sobrinos. 


\section{Agradecimentos:}

Aos meus pais e irmàos que sempre me incentivaram a estudar e que ainda estando longe me deram a força necessária para chegar atê aqui.

Ao meu orientador, o professor Artur H. Tomita, pelo apoio e paciência constantes.

A professora Elza Gomide, pelo apoio na preparação dos exames de qualificação.

Aos proferssores Avivah Gilboy, Julio Borghi e Artigas Barreiro de Uruguai, porque com eles aprendí a estudar matemática.

As minhas amigas da graduação: Paula, Margui, Patricia, Ximena, Andrea.

A Marcelo e Jorge que me receberam de braços abertos em São Paulo. A Cecilia, Jorge, Fernando, Daniela, Irene, Nelson, Marquitos que também formaram parte da minha família paulista.

Aos meus amigos da pos-graduação: Barbara. Raul, Daniel, Samuel, Roberto, Sidney, Fernando, Marcio, Claus, Liane, Fernando, Regina.

Aos meus amigos de sempre: "la barra de la escuela 180 y del liceo 20 " e Laura Castro, claro.

Ao Ze, amigo de todas as horas, pela paciência e a força sem as quais todo teria sido mais difícil. 


\section{Sumário}

$\begin{array}{ll}\text { Introdução } & 3\end{array}$

1 Preliminares $\quad 6$

1.1 Conceitos básicos . . . . . . . . . . . . . . . 6

1.2 Limite Inverso . . . . . . . . . . . . . . . . . . . . . . . . . . . 14

2 Conjuntos de pontos fixos em espaços métricos e não métricos 17

2.1 Produto de espaços metrizáveis . . . . . . . . . . . . . . 18

2.2 Exemplos em espaços não métricos . . . . . . . . . . . . 20

2.3 Condições suficientes para espaços produto . . . . . . . . . . . . 29

2.4 Resultados independentes . . . . . . . . . . . . . . 43

3 A CIP em produtos não enumeráveis de espaços lineares $\quad 46$

3.1 Algumas definições . . . . . . . . . . . . . . . . . . . 46

3.2 Lemas Geométricos . . . . . . . . . . . . . . . . . . . . . . . . 48

3.3 Construção de um conjunto splitting . . . . . . . . . . . . . 54 
4 Conjuntos de pontos fixos de homeomorfismos : dois exemplos $\quad 60$ 4.1 CIPH no cubo de Hilbert . . . . . . . . . . . . . . . . 60

4.2 CIPH no cubo de Cantor . . . . . . . . . . . . . . 65

$\begin{array}{ll}\text { Apêndice } & 79\end{array}$

Referências Bibliográficas $\quad 85$ 


\section{INTRODUÇÃ O}

Um subconjunto $K$ de um espaço $X$ é chamado conjunto de pontos fixos de $X$ se existe uma funçào contínua $f: X \longrightarrow X$ tal que $f(x)=x$ se e somente se $x \in K$. Em 1967 H.Robbins [Rob67] trabalhou com conjuntos de pontos fixos de funções contínuas e de homeomorfismos na bola fechada $B^{n}$ de dimensão $n$. Embora não foi possível chegar a uma condição necessaria e suficiente para que um subconjunto $A$ de $B^{n}$ seja conjunto de pontos fixos de um homeomorfismo, Robbins mostrou que o problema tem uma solucão simples para o caso das funções contínuas: a CIP ("complete invariance property" ou "propriedade da invariância completa"). O nome de CIP foi dado por L.E. Ward em 1973 [Wari3] à seguinte propriedade:

Um espaço X' tem a CIP se todo subconjunto fechado não vazio de $X$ é um conjunto de pontos fixos de $X^{\prime}$.

A pesquisa a respeito da CIP centrou-se em dois tópicos:

Quais espaços tem a CIIP? ;

Como se comporta a CIP com relaçào as construções geométricas? (por exemplo: produtos cartesianos).

Em [War73], Ward mostrou que todo subconjunto convexo $X$ de um espaço linear normado tem CIP. Em 1979 J.R. Martin e S.B. Nadler Jr. provaram que todo grupo topológico metrizável localmente compacto tem CIP. Temos também um resultado de $n$-variedades publicado por H. Shirmer em 1981 [Sch81] que diz que toda $n$-variedade topológica compacta, com ou sem bordo tem CIP. Estes são, entào, algums espaços com CIP.

Quanto ao comportamento da propriedade com relação ao produto de espaços, em [Sch81], Shirmer mostra a existência de um espaço $X^{\prime}$ com CIP tal que o produto $X \times I$ não tem CIP. $(I=[0,1])$.

Para o problema de determinar em que condiçòes um subconjunto $A$ da bola $B^{n}$ é o conjunto de pontos fixos de um homeomorfismo, em 1968 [Sch69] Shirmer deu 
algumas respostas:

(i) Se $n$ é par, todo subconjunto fechado nào vazio $A$ de $B^{n}$ é o conjunto de pontos fixos de um homeomorfismo $f: B^{n} \longrightarrow B^{n}$ i.e. $B^{n}$ tem a CIPH (complete invariance property with respect to homeomorphisms).

(ii) Se $n$ é impar a soluçào depende de $A \cap \partial B^{n}=\emptyset$ ou $A \cap \partial B^{n} \neq \emptyset$.

Sabemos que se $n$ é impar, então $B^{n}$ não tem a CIPH. Robbins [Rob67] mostrou a existência de um subconjunto fechado de $B^{2 n+1}$ que não é conjunto de pontos fixos de nenhum homeomorfismo $f: B^{2 n+1} \longrightarrow B^{2 n+1}$.

Este trabalho començou com o estudo em profundidade do artigo [Kos92] que demonstra que o cubo de Tychonoff nào tem CIP. Foi esse nosso primeiro contato com esta propriedade. Na introdução de [Kos92] se fez referência 'a CIP no cubo de Hilbert. Surge entào uma pergunta: o que faz que a CIP funcione no cubo de Hilbert e não no cubo de Tychonoff?.Obviamente o fato do primeiro espaço ser métrico e o segundo nào, deve ter alguma coisa a ver. Em busca de respostas recorremos ao artigo [MW84] oncle a existência da CIP em espaços métricos, espaços não métricos e espaços produto é analizada. A seguir consideramos o cubo de Hilbert e o cubo de Cantor, ambos espaços com CIP, um métrico e outro não métrico. Será que a propriedade se conserva para homeomorfismos?. Achamos a resposta relativa ao cubo de Hilbert no artigo [Mar88], e a resposta a respeito do cubo de Cantor no artigo [CN9T]

No primeiro capítulo fazemos um ressumo dos conceitos e propriedades conjuntísticas e topológicas que serão úteis nos capítulos seguintes.

No segundo capítulo començamos a abordar a propriedade da invariância completa em espaços produto. Vemos por exemplo que a reta longa (L) e a reta de Sorgenfrey $(\mathrm{K})$ tem CIP, porém $L \times L$ e $K \times K$ não tem. logo a CIP não é preservada por auto-produtos. Agora. em que condições um espaço produto tem CIP?. Temos dois tipos de espaços produto a ser considerados. Por um lado, apresentamos 
condições suficientes para o produto enumerável de espaços métricos ter CIP, e por outro condiçòes suficientes para um produto infinito de espaços ter CIP.

Vemos também que $3 \mathbb{N}$ (compactificado de Stone Čech do conjunto dos inteiros N) não tem CIP, emquanto que $(\beta \mathbb{N})^{\mathfrak{c}}$ tem, onde $\mathfrak{c}$ denota o cardinal do contínuo.

Por último, vemos que se $K$ é a reta de Sorgenfrey, $K^{\lambda}$ tem CIP para todo cardinal $\lambda \geq \mathfrak{c}$ e que o fato de $K^{\omega_{1}}$ ter ou não ter CIP é independente de (ZFC).

O terceiro capítulo é dedicado a mostrar que se $\kappa$ é um cardinal infinito não enumerável e para cada $\alpha<\kappa, X_{\alpha}$ é um subespaço compacto e convexo de algum espaço linear normado, então $\prod_{\alpha<\kappa} X_{\alpha}$ não tem CIP. Em particular, o cubo de Tychonoff não tem CIP.

No quarto capítulo nos concentramos, em dois teoremas:

- o cubo de Hilbert tem CIPH;

- o cubo de Cantor tem CIPH. 


\section{Capítulo 1}

\section{Preliminares}

\subsection{Conceitos básicos}

Apresentamos neste capítulo algumas definições e propriedades básicas de Teoria dos conjuntos e de Topologia Geral, que seram usados com familiaridade nos capítulos seguintes.

Teoria dos conjuntos:

A referência utilizada para os conceitos conjuntistas é o livro do Kunen [Kun80]. Trabalharemos no contexto da axiomática de Zermelo-Fraenkel com o Axioma da Escolha (ZFC).

Denotaremos a diferência entre dois conjuntos $A$ e $B$ por $A-B$.

Se $f: X \longrightarrow Y$ é uma função, usaremos as seguintes notações: $\operatorname{dom}(f)$ para o dominio da função, e $i m(f)$ para a imagem. Se $A \subseteq X$ e $B \subseteq Y$ então $f(A)$ indica a imagem de $A$ por $f$, e $f^{-1}(B)$ a pre-imagem de $B$ por $f$. A restrição da função $f$ ao conjunto $A$ será denotada por $\left.f\right|_{A}$, ou seja que $\left.f\right|_{A}: A \longrightarrow Y$ e $\left.f\right|_{A}(x)=f(x) \forall x \in A$.

Um conjunto A é dito transitivo se cada elemento de A é também um subconjunto de $A$. Um conjunto Á um ordinal se é transitivo e bem ordenado por $\epsilon$. Observamos 
que os elementos de um ordinal sào também ordinais. Usaremos as letras gregas $\alpha$ 3. $\gamma, \delta, \mu$ para designar ordinais. Se $\alpha$ e $\beta$ sào ordinais e $\alpha \in \beta$ dizemos que $\alpha<\beta$ e estas duas expresòes são equivalentes.

Se $X^{\prime}$ é um conjunto de ordinais, então $\sup \left(X^{r}\right)=\cup X^{\prime}$ e se $X \neq \emptyset, \min (X)=\cap X^{\prime}$.

Seja $\alpha$ um ordinal. Se define $S(\alpha)$ ou $\alpha+1$ como o ordinal $\alpha \cup\{\alpha\}$ chamado ordinal sucessor de $\alpha$. Um ordinal $\alpha$ é dito ordinal sucessor se existe um ordinal $\beta$ tal que $\alpha=S(\beta)$; e $\alpha$ é dito ordinal limite se $\alpha \neq 0$ e $\alpha$ nào é sucessor.

Dois conjuntos $A$ e $B$ são equipotentes se existe uma função $f: A \longrightarrow B$ bijetora. Se $f$ é um conjunto bem ordenado, se define a cardinalidade de $A(|A|)$ como o menor ordinal a equipotente com A. Sob o Axioma da Escolha, todo conjunto é bem ordenado e portanto $|A|$ é definido para todo conjunto $A$. A cardinalidade do conjunto dos números naturais é $\omega$, o menor ordinal limite. Um cardinal é um ordinal $\alpha$ tal que $\alpha=|\alpha|$, i.e. $\alpha$ não é equipotente a nenhum ordinal menor que o próprio $\alpha$. Usaremos as letras gregas $\lambda, \kappa$ para designar cardinais.

Se $\lambda$ é um cardinal, $\lambda^{+}$é o menor cardinal maior que $\lambda$. Dizemos que $\kappa$ é um cardinal sucessor se $\kappa=\lambda^{+}$para algum cardinal $\lambda ; \kappa$ é um cardinal limite se $\kappa>\omega$ e nào é um cardinal sucessor. Define-se

$$
\begin{aligned}
& \text { (i) } \omega_{0}=\omega \\
& \text { (ii) } \omega_{\alpha+1}=\left(\omega_{\alpha}\right)^{+} \\
& \text {(iii) para } \gamma \text { limite, } \omega_{\gamma}=\sup \left\{\omega_{\alpha}: \alpha<\gamma\right\}
\end{aligned}
$$

E temos as seguintes propriedades (cap1, 10.19 [Kuns0]):

(i) Cada $\omega_{\alpha}$ é um cardinal,

(ii) Cada cardinal infinito é igual a $\omega_{\alpha}$, para algum ordinal $\alpha$,

(iii) Se $\alpha<\beta$, entào $\omega_{\alpha}<\omega_{\beta}$,

(iv) $\omega_{\alpha}$ é cardinal limite (sucessor) sse $\alpha$ é ordinal limite (sucessor).

Se $\mathcal{A}$ é um conjunto e $\lambda$ um cardinal, se definen os seguintes conjuntos:

$$
[A]^{\lambda}=\{B \subseteq A:|B|=\lambda\} \quad \text { e } \quad[A]^{<\lambda}=\{B \subseteq A:|B|<\lambda\}
$$


A Hipòtese do Contínuo $(\mathrm{CH})$ é a asserçào $2^{\omega}=\omega_{1}$. Usaremos também a notaçào $\mathrm{c}$ para $2^{\omega}$.

Sejam $a .3$ ordinais. Uma função $f: \alpha \longrightarrow \beta$ é cofinal em $\beta$ se $i m(f)$ é nào limitada em 3 i.e. se $\sup (\operatorname{im}(f))=\beta$. A cofinalidade de $\beta(c f(\beta))$ é o menor ordinal $\alpha$ tal que existe uma funçào cofinal $f: \alpha \longrightarrow \beta$. Então $c f(\beta) \leq \beta$ e se $\beta$ é sucessor $c f(\beta)=1$. L'm ordinal $\beta$ é dito regular se é ordinal limite e $c f(\beta)=\beta$. Valem os seguintes fatos respeito da cofinalidade:

(i) se $\alpha$ é regular entào $\alpha$ é um cardinal,

(ii) $\kappa^{+}$é regular para todo cardinal infinito $\kappa$,

(iii) se $\alpha$ é um ordinal limite, então $c f\left(\omega_{\alpha}\right)=c f(\alpha)$.

Se $\mu$ é um ordinal limite, um conjunto $C \subset \mu$ é fechado em $\mu$ sse para todo ordinal limite $\delta<\mu$. se $C \cap \delta$ é não limitado em $\delta$ então $\delta \in C$. C é c.u.b em $\mu$ sse $C$ é fechado e nào limitado em $\mu$ (sse $C$ é fechado e $\sup (C)=\mu$ ). Tem-se o seguinte resultado respeito aos conjuntos c.u.b:

Se $\lambda=c f(\mu)>\omega$. então a interseç̧ão de qualquer família de menos do que $\lambda$ c.u.b's em $\mu$ é também c.u.b em $\mu$.

Se $c f(\mu)>\omega$. um conjunto $S \subset \mu$ é estacionário em $\mu$ se $S \cap C \neq \emptyset$ para cada conjunto $C$ c.u.b em $\mu$.

Pressing-down lemma (cap2, 6.15 [Kuns0]): Sejam $\kappa>\omega$ um cardinal regular. $S \subseteq \kappa$ um conjunto estacionário e $f: S \longrightarrow \kappa$ uma função tal que $f(\gamma)<\gamma \forall \gamma \in S$ : então existe $\alpha<\kappa$ tal que $f^{-1}(\{\alpha\})$ é estacionário.

$M A(\kappa)$ é a asserção:

Se $(\mathbb{P}, \leq)$ é uma ordem parcial não vazi, c.c.c, e $\mathcal{D}$ é uma família de $\leq \kappa$ subconjuntos densos de $\mathbb{P}$. então existe um filtro $G$ em $\mathbb{P}$ tal que $\forall D \in \mathcal{D}(G \cap D \neq \emptyset)$.

O Axioma de Martin (MA) é a asserção : $\forall \kappa<2^{\omega}(M A(\kappa))$.

A respeito do Axioma de Martin, o seguinte resultado será muito útil:

$\mathrm{MA}(\kappa) \Rightarrow 2^{\kappa}=2^{\omega}$. 


\section{Topologia Geral:}

Foram utilizados como referências básicas para os conceitos topológico o livro de Engelking [Eng89] e as Notas de aula [TJ97].

Um espaço topológico é um par $\langle X, \tau\rangle$, onde $X$ é o conjunto-suporte e $\tau$ a topologia definida no conjunto $X$. Quando no houver dúvidas respeito a topologia nos referiremos ao espaço $\langle X, \tau\rangle$ por espaço topológico $X$ ou espaço $X$.

Assumiremos conhecidos os conceitos básicos da Topologia Geral, como por exemplo: conjunto aberto, conjunto fechado, vizinhanças de um ponto, base para o espaço. base para um ponto, fecho de um conjunto, axiomas de enumerabilidade e separaçào (espaços $T_{i}$ para $i=0.1,2,3,3 \frac{1}{2}, 4$ ). Assumiremos que os espaços $T_{i}$ são $T_{1}$ para todo $i \geq 1$.

Se $A$ é um subconjunto do espaço $X$, denotamos por $C l_{X}(A)$ ou $\bar{A}$ o fecho de $A$ em $X$.

Usaremos tembém os termos Hausdorff para $T_{2}$, regular para $T_{3}$, Tychonoff e completamente regular para $T_{3 \frac{1}{2}}$, normal para $T_{4}$.

Um subconjunto fechado de um espaço $X$ é dito um $G_{\delta}^{\prime}$ se é a interseção de uma família enumerável de abertos de $X$. Um subconjunto aberto de um espaço $X$ é dito um $F_{\sigma}$ se é a união de uma família enumerável de fechados de $X$.

Vale a seguinte propriedade respeito a espaços normais:

Um subconjunto A de um espaço normal $X$ é um fechado $G_{\delta}^{\prime}$ (aberto $F_{\sigma}$ ) se e somente se existe uma funçào contínua $f: X \rightarrow I$ tal que $\left.\left.A=f^{-1}(0)\left(A=f^{-1}(] 0,1\right]\right)\right)$, onde I é o intervalo real $[0,1]$.

Um subconjunto $A$ de um espaço $X$ é dito zero-set (cozero-set) se $A=f^{-1}(0)$ $\left.\left.\left(A=f^{-1}(] 0,1\right]\right)\right)$ para alguma função contínua $f: X \longrightarrow I$.

Pela propriedade anterior temos que num espaço normal, zero-sets (cozero-sets) coincidem com os $G_{r_{\delta}}\left(F_{\sigma}\right)$.

Um espaço X. $T_{1}$, é completamente regular se e somente se a família de todos os 
cozero-sets do espaço formam uma base para $X$. Um espaço topológico $X$ é perfeitamente normal se $X$ é um espaço normal e todo subconjunto fechado de $X$ é um $G_{\delta}$ (ou se todo aberto é um $F_{\sigma}$ ). Temos o seguinte teorema respeito de espaços perfeitamente normais:

Teorema de Vedenissoff:

Para todo espaço $T_{1}$ as seguintes condições são equivalentes:

(i) O espaço X' é perfeitamente normal,

(ii) subconjuntos fechados de $X$ são zero-sets,

(iii) subconjuntos abertos de $X$ são cozero-sets,

(iv) para cada par de subconjuntos fechados disjuntos $A, B \subset X$ existe uma função contínua $f: X \longrightarrow I$ tal que $f^{-1}(0)=A$ e $f^{-1}(1)=B$.

Seja $X$ um conjunto linearmento ordenado por $<$, contendo no mínimo dois pontos. Para $a, b \in X$ satisfazendo $a<b$ sejam

$$
\begin{aligned}
] a, b[ & =\{x \in X: a<x<b\} \\
] \leftarrow, a[ & =\{x \in X: x<a\} \\
] a, \rightarrow[ & =\{x \in X: a<x\}
\end{aligned}
$$

Os conjutos acima definidos são chamados intervalos em $X$. A família de todos os intervalos em $X$ são uma base de abertos para uma topologia. Esta topologia se denomina topologia da ordem em $X$. Algums exemplos de topologia da ordem são:

Reta longa (Long line) : Seja $W_{0}$ o conjunto de todos os ordinais enumeráveis. No conjunto $V_{0}=W_{0} \times[0,1[$ consideramos a ordem linear $<$ definida por:

$$
\left(\alpha_{1}, t_{1}\right)<\left(\alpha_{2}, t_{2}\right) \text { se } \alpha_{1}<\alpha_{2} \text { ou } \alpha_{1}=\alpha_{2} \text { e } t_{1}<t_{2}
$$

$\mathrm{O}$ conjunto $V_{0}$ com a topologia induzida por $<$ é chamado de reta longa $(\mathbf{L})$.

Segmento longo (Long segment): Seja $V=V_{0} \cup\left\{\omega_{1}\right\} \operatorname{com} x<\omega_{1} \forall x \in V_{0}$. $\mathrm{O}$ conjunto $V$ com a topologia induzida pela ordem linear $<$ é chamado de long segment $\left(\mathbf{L}^{*}\right)$.

Para cada $x_{0} \in V_{0}$, o subespaço $M=\left\{x \in V_{0}: x \leq x_{0}\right\}$ de $V_{0}$ é homeomorfo ao 
intervalo $[0,1]$. (ver exemplo 3.12.19 [Eng89]).

Se $f$ é uma funçào contínua, definida de um espaço $X^{\prime}$ num espaço de Hausdorff $Y$, entào $f$ está determinada pelos valores num subconjunto denso de $X$.

Esta propriedade e consecuência da seguinte proposição:

Sejam D um subespaço denso de X ef uma funçào contínua de $D$ num espaço de Hausdorff $Y$. Entào se $f$ pode ser estendida para $X$, a extensão é única.

Se $\left\{X_{s}: s \in S\right\}$ é uma família de conjuntos, então o produto cartesiano desta família será o conjunto de todas as funções $f: S \longrightarrow \bigcup_{s \in S} X_{s}$ tal que para cada $s \in S$ temos $f(s) \in X_{s}$.

Seja $\mathcal{F}=\left\{X_{s}: s \in S\right\}$ uma família de espaços topológicos. A topologia gerada pela base

$$
\begin{aligned}
& \left\{\pi_{s_{1}}^{-1}\left(U_{s_{1}}\right) \cap \ldots \cap \pi_{s_{k}}^{-1} U_{s_{k}}: s_{1} \ldots s_{k} \in S \text { e } U_{s_{j}} \text { é aberto de } X_{s_{\jmath}}\right\}= \\
= & \left\{\prod_{s \in S} U_{s}: U_{s} \text { é aberto de } X_{s} \text { e }\left\{s \in S: U_{s} \neq X_{s} \text { é finito }\right\}\right\}
\end{aligned}
$$

é o produto de Tychonoff da família $\mathcal{F}$ e denotado por $\prod_{s \in S} X_{s}$.

Seja $X$ um espaço topológico e $\left\{Y_{s}: s \in S\right\}$ uma família de espaços topológicos. Para cada $s \in S$ seja $f_{s}: X \longrightarrow Y_{s}$ uma função contínua. Definimos a função comtínua $\triangle_{s \in S} f_{s}: X \longrightarrow \prod_{s \in S} Y_{s}$ por $\triangle_{s \in S} f_{s}(x)=\left(f_{s}(x)\right)_{s \in S} \forall x \in X$. Chamamos esta função de função diagonal.

Um espaço topológico $X$ é chamado zero-dimensional se $X$ é um espaço $T_{1}$ não vazio e possui uma base de conjuntos abertos-e-fechados (clopens). Todo espaço zerodimensional é completamente regular.

Um exemplo de espaço zero-dimensional é a Reta de Sorgenfrey que denotaremos por $K$. Este espaço tem como conjunto suporte os números reais. Um subconjunto $A$ de $K$ é um aberto na topologia da Reta de Sorgenfrey sse para cada ponto $x \in A$ existe um $\Sigma>0$ tal que $[x, x+\Sigma[$ está contido em $A$.

A família $\mathcal{F}=\{[x, x+\varepsilon[: x \in K$ e $\varepsilon>0\}$ formam uma base de clopens para $K$.

Um espaço $X$ é dito conexo se não existem subconjuntos abertos não vazios $U$ e 
$V$ de $X$ tais que $U \cap V=\emptyset$ e $U \cup V=X$. Os seguintes teoremas serão muito úteis neste trabalho:

(1) Seja f uma funçào contínua de um espaço X em um espaço $Y$. Se A é um subconjunto conexo de $X$, então $f(A)$ é um subconjunto conexo de $Y$.

(2) Se um subespaço $C$ de um espaço $X$ é conexo, então para cada par $X_{1}, X_{2}$ de subconjuntos de $X$ tais que $X_{1} \cap \overline{X_{2}}=\emptyset, \overline{X_{1}} \cap X_{2}=\emptyset$ e $\subset X_{1} \cup X_{2}$, temos que $C \subset \mathrm{X}_{1}$ ou $C \subset \mathrm{X}_{2}$.

A componente conera de um ponto $x$ num espaço $X$ é a união de todos os subespaços conexos de $X^{\prime}$ que contém o ponto $x$. As componentes conexas de dois pontos diferentes de $X^{\prime}$ coincidem ou são disjuntas.

A quase-componente de um ponto $x$ num espaço $X$, é a interseção de todos os subconjuntos abertos-e-fechados de $X$ que contém o ponto $x$. As quase-componentes de dois pontos distintos de $X$ coincidem ou são disjuntas. Um espaço $X$ é dito totalmente desconero se a quase-componente de cada ponto $x \in X^{\prime}$ é o conjunto unitário $\{x\}$. Todo espaço zero-dimensional é totalmente desconexo.

Seja $X$ um espaço topológico, $A \subseteq X, x \in X$. O ponto $x$ é chamado um ponto de acumulação completo de $A$ se para toda vizinhança aberta $U$ de $x$ vale que $|U \cap A|=|A|$.

Para um espaço $X$ sào equivalentes:

(i) X' é compacto,

(ii) toda família de subconjuntos fechados com a propriedade da interseção finita em $X$ tem interseção não vazia,

(iii) todo subconjunto infinito de $X$ possui ponto de acumulação completo.

Um espaço topológico $\langle X, \tau\rangle$ é metrizável se existe uma métrica $\rho$ definida em $X$ tal que a topologia induzida por $\rho$ em $X$ coincide $\operatorname{com} \tau$. $\rho$ é chamada métrica no espaço $\mathrm{X}$.

Duas métricas $\rho_{1}, \rho_{2}$ definidas num conjunto $X$ sào equivalentes se induzem a mesma 
topologia em $X^{\prime}$. Se $(X . \rho)$ é um espaço métrico, entào existe uma métrica $\rho_{1}$ em $X$ que é equivalente com $\rho$ e é limitada por 1 .

As seguintes propriedades referentes a espaço métricos serão de grande utilidade:

Um espaço compacto $X$ é metrizável se e somente se a diagonal $\Delta=\left\{(x, x): x \in X^{\prime}\right\}$ é um $G_{\delta}$ no produto cartesiano $X \times X$. (ver execício 4.2.B do Engelking)

$O$ produto cartesiano $M \times Y$ de um espaço métrico $M$ e um espaço perfeitamente normal $Y$, é perfeitamente normal. (ver exercício 4.5.16 do Engelking)

Um espaço topológico $X^{\prime}$ é chamado homogêneo se para todo par de pontos $x, y \in$ $X$, existe um homeomorfismo $h: X \rightarrow X \operatorname{com} h(x)=y$.

Um exemplo de espaço homogêneo é o Cubo de Hilbert que é o produto cartesiano de $\omega$ cópias do intervalo real $[-1,1]$.

Usaremos as seguintes funçòes cardinais:

$$
\begin{aligned}
\text { peso } & w(X)=\min \left\{|\mathcal{B}|: \mathcal{B} \text { é uma base para } X^{\prime}\right\}+\omega \\
\text { densidade } & d\left(X^{\prime}\right)=\min \left\{\left|S^{\prime}\right|: S \subseteq X, \bar{S}=X^{\prime}\right\}+\omega
\end{aligned}
$$

Teorema (Hewitt, . Marzewski, Pondiczery) ([Hod84])

Se $X=\prod_{t \in T} X_{t}$. onde $|T| \leq 2^{\kappa} \in d\left(X_{t}\right) \leq \kappa$ para todo $t \in T$, então $d(X) \leq \kappa$.

$\mathbb{N}$ representa o conjunto dos números naturais com a topologia discreta. $\beta \mathbb{N}$ é o compactificado de Stone-Čech do espaço $\mathbb{N}$.

Seja $S$ o conjunto de todos os ultrafiltros em $\mathbb{N}$. Para cada conjunto $E \subseteq \mathbb{N}$, seja $V(E)$ o conjunto de todos os ultrafiltros em $\mathbb{N}$ aos quais $E$ pertence. Consideramos a topologia em $S$ gerada pela base $\{V(E): E \subseteq \mathbb{N}\}$. उ $\mathbb{N}$ é homeomorfo ao espaço $S$. (ver [Rud.56])

As seguintes são algumas propriedades do espaço $\beta \mathbb{N}$ as quais faremos referência no tabalho:

(i) $V(\mathbb{N}-E)=3 \mathbb{N}-V(E) \quad \forall E \subseteq \mathbb{N}$, portanto $\{V(E): E \subseteq \mathbb{N}\}$ é uma base de clopen e $3 \mathbb{N}$ é um espaço zero-dimensional. ([Rudj56] 
(ii) Todo subconjunto fechado infinito de $3 \mathbb{N}$ contém um subconjunto homeomorfo a $3 \mathbb{N}$. ([Engs9]

(iii) $d(3 \mathbb{N}-\mathbb{N})=\mathfrak{c} .([\operatorname{Hod} 84])$

Por $\mathbb{R}$ denotamos o conjuntos dos números reais e por $\mathbb{C}$ o conjunto dos números complexos.

\section{$1.2 \quad$ Limite Inverso}

Em mais de uma demonstração vamos o usar sistemas inversos e limites inversos para construir conjuntos em espaços produto ou funções entre espaços produto. Fazemos nesta seção um pequeno resumo das propriedades de limite inverso que serão úteis nos capítulos seguintes.

Seja $X$ um conjunto e $\leq$ uma relação em $X$. Dizemos que $X$ é dirigido por $\leq$ se possui as seguintes propriedades:

(1) se $x \leq$ e $y \leq z$, entào $x \leq z$,

(2) para cadla $x \in X, x \leq x$,

(3) para todo $x, y \in X$, existe $z \in X$ tal que $x \leq z$ e $y \leq z$.

Seja $\Sigma$ um conjunto dirigido pela relaçào $\leq$. Suponhamos que a cada $\sigma \in \Sigma$ corresponde um espaço topológico $X_{\sigma}^{\prime}$, e que para cada par $\sigma, \rho \in \Sigma$ satisfazendo $\rho \leq \sigma$ està definida uma função contínua $\pi_{\rho}^{\sigma}: X_{\sigma} \longrightarrow X_{\rho}$. Suponhamos também que $\pi_{\tau}^{\rho} \pi_{\rho}^{\sigma}=\pi_{\tau}^{\sigma} \forall \sigma, \rho, \tau \in \Sigma$ satisfazendo $\tau \leq \rho \leq \sigma$, e que $\pi_{\sigma}^{\sigma}=i d_{X_{\sigma}} \forall \sigma \in \Sigma$. Nestas condições dizemos que a família $S=\left\{X_{\sigma}, \pi_{\rho}^{\sigma}, \Sigma\right\}$ é um sistema inverso dos espaços $X_{\sigma}$.

Seja $S=\left\{X_{\sigma}, \pi_{\rho}^{\sigma}, \Sigma\right\}$ um sistema inverso. Um elemento $\left\{x_{\sigma}\right\}$ do produto cartesiano $\prod_{\sigma \in \Sigma} X_{\sigma}$ é chamado thread de $S$ se $\pi_{\rho}^{\sigma}\left(x_{\sigma}\right)=x_{\rho} \forall \sigma, \rho \in \Sigma$ satisfazendo $\rho \leq \sigma$. O subespaço formado por todos os threads de $S$ é chamado limite inverso de $S$ e é denotado por $\overleftarrow{\lim } S$ ou $\overleftarrow{\lim }\left\{X_{\sigma}, \pi_{\rho}^{\sigma}, \Sigma\right\}$ 
Neste trabalho o conjunto dirigido $\Sigma$ va a ser em todos os casos um ordinal limite. Temos entào que se $\mu$ é nosso conjunto dirigido e $\alpha \leq \beta \in \mu, \alpha \subset \beta$, logo $X_{\beta}=\prod_{\alpha \in \beta} X_{\beta}$ para cada $\beta \in \mu$. Temos também que para cada par $\alpha, \beta \in \mu$ satisfazendo $\alpha \leq \beta$. as funçòes $\pi_{\alpha}^{\beta}: X_{\beta} \longrightarrow X_{\alpha}$ são a restrição dos elemetos de $X_{\beta}$ ao ordinal $\alpha$.

Seja $S=\left\{X_{\beta}, \pi_{\alpha}^{3}, \mu\right\}$ um sistema inverso e seja $X=\overleftarrow{\text { iim }} S$. Para cada $\beta<\mu$ a funçào contínua $\pi_{3}: X \longrightarrow X_{\beta}$ é a projeçào $\prod_{\alpha \in \mu} X_{\alpha} \longrightarrow X_{\beta}$. Se $\alpha, \beta \in \mu \operatorname{com} \alpha \leq \beta$, as projeções $\pi_{\alpha}$ e $\pi_{3}$ satisfazem a igualdade $\pi_{\alpha}=\pi_{\alpha}^{\beta} \pi_{\beta}$.

Proposição Seja $S=\left\{X_{\beta}, \pi_{\alpha}^{\beta}, \mu\right\}$ um sistema inverso e $\mathrm{X}=\stackrel{\leftarrow}{\text { iim }} S$. Para cada subconjunto A de $X^{\prime}$ a família $S_{A}=\left\{\overline{A_{\beta}}, \tilde{\pi}_{\alpha}^{\beta}, \mu\right\}$ onde $A_{\beta}=\pi_{\beta}(A)$ e $\tilde{\pi}_{\alpha}^{\beta}(x)=\pi_{\alpha}^{\beta}(x)$ para $x \in \overline{A_{\beta}}$, é um sistema inverso e $\stackrel{\mathrm{im}}{S_{A}}=\bar{A} \subset \mathrm{X}$.

Corolário Todo subespaço fechado $A$ do limite inverso de $S=\left\{X_{\beta}, \pi_{\alpha}^{\beta}, \mu\right\}$ é o limite inverso do sistema inverso $S_{A}=\left\{\overline{A_{\beta}}, \tilde{\pi}_{\alpha}^{\beta}, \mu\right\}$ de subespaços fechados $\overline{A_{\beta}}$ de $X_{\beta}$.

Sejam dois sistemas inversos $S=\left\{X_{\beta}, \pi_{\alpha}^{3}, \mu\right\}$ e $S^{\prime \prime}=\left\{Y_{\beta}, \hat{\pi}_{\alpha}^{\beta}, \mu\right\}$. Uma aplicação de $S$ em $S^{\prime \prime}$ é uma familia $\left\{f_{\beta}\right\}$ de funçòes contínuas $f_{\beta}: X_{\beta} \longrightarrow Y_{\beta}$ definida para cada $\beta \in \mu$ tais que :

$$
\hat{\pi}_{\alpha}^{\beta} f_{\beta}=f_{\alpha} \pi_{\alpha}^{\beta} \quad \forall \alpha, \beta \in \mu \operatorname{com} \alpha \leq \beta
$$

ou seja que o seguinte diagrama comuta

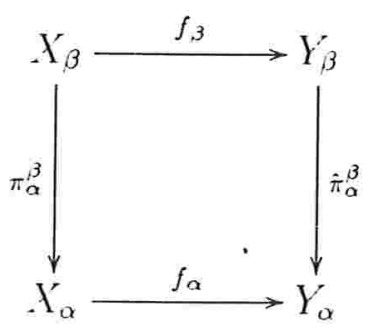

Toda aplicação de um sistema inverso $S$ num sistema inverso $S^{\prime \prime}$ induz uma função $f: \overleftarrow{\lim } S \longrightarrow \overleftrightarrow{\lim } S^{\prime}$. Esta função $f$ se define da seguinte forma: seja $X=\stackrel{\leftarrow}{\mathrm{im}} S$ e seja $x=\left\{x_{\beta}\right\}_{3 \in \mu}$ um elemento de $X$. Entào $f(x)=y$ onde $y=\left\{y_{\beta}\right\}_{\beta \in \mu}$ e $y_{\beta}=f_{\beta}\left(x_{\beta}\right)$ para cada $\beta \in \mu$. A função $f$ assim definida é continua e é chamada função limite de 
$\left\{f_{3}\right\}$ denotada por $\overleftarrow{\lim } f_{3}$

Proposição Seja $\left\{f_{\beta}\right\}_{\beta \in \mu}$ uma função do sistema inverso $S=\left\{X_{\beta}, \pi_{\alpha}^{\beta}, \mu\right\}$ no sistema inverso $S^{\prime \prime}=\left\{Y_{\beta}, \hat{\pi}_{\alpha}^{\beta}, \mu\right\}$. Se todas as funções $f_{\beta}: X_{\beta} \longrightarrow Y_{\beta}$ são homeomorfismos. então a funçào limite $f=\lim f_{\beta}$ é também um homeomorfismo. 


\section{Capítulo 2}

\section{Conjuntos de pontos fixos em espaços métricos e não métricos}

Neste capítulo sào dadas condições que assegurem que certos espaços e grupos topológicos tenham a C'IP, e também sào dados exemplos de espaços produto e grupos que não tem ('IP.

Em particular se mostrará que a CIP nào é preservada por auto-produtos. Por exemplo, veremos que a long-line $L$ tem CIP enquanto que $L \times L$ nào.

Também vamos ver que o produto $K^{-\lambda}$ de $\lambda$ cópias da reta de Sorgenfrey $K$, tem CIP se $\lambda=1$ ou $\lambda=2^{\omega_{0}}$. porém não tem se $1<\lambda \leq \omega_{0}$.

No caso de $\beta \mathbb{N}$. se mostrará que $(\beta \mathbb{N})^{\mathfrak{c}}$ tem CIP mas $\beta \mathbb{N}$ nào, onde $\mathfrak{c}$ indica a cardinalidade do contínuo.

Por último, veremos exemplos de espaços topológicos nos quais a existência da CIP nào pode ser provada com os axiomas usuais da teoria de conjuntos ( $\mathrm{ZFC}$ ), mas mostramos que axiomas adicionais podem ser escolhidos de forma que impliquem a existência da C'IP nesses espaços. 


\subsection{Produto de espaços metrizáveis}

Definição 2.1 Seja X um espaço topológico. Chamamos deformação a uma função de homotopia $H: X^{\prime} \times I \longrightarrow X^{\prime}$ tal que $H(x, 0)=x \quad \forall x \in X^{\prime}$.

Definição 2.2 Um espaço $X$ tem a propriedade $W$ se para cada ponto $p \in X$ existe uma deformação $H: X \times I \longrightarrow X$ tal que $H(x, t) \neq x$ se $x \neq p$ e $t>0$.

Se $X$ satisfaz a condiçào $H(x, t) \neq x$ sempre que $t>0$, dizemos que $X$ tem a propriedade $W$ (forte)

Observação 2.3 Se X'é um espaço topológico com a propriedade $W$ (forte) e se $Y$ é um espaço qualquer, então $X \times Y$ tem a propriedade $W$ (forte).

prova: Seja $H: X^{\prime} \times I \longrightarrow X$ uma deformação tal que $H(x, t) \neq x \forall t>0$.

Definimos $H^{\prime}:(X \times Y) \times I \longrightarrow X \times Y$ como segue:

$$
H^{\prime}((x, y), t)=(H(x, t), y) \forall t>0
$$

De igual forma pode-se provar que se $\mathcal{F}=\left\{X_{s}: s \in S^{\prime}\right\}$ é uma família de espaços topológico e se para algum $s_{0} \in S, X_{s_{0}}$ tem a propriedade $W$ (forte), então o espaço produto $\prod\left\{X_{s}: s \in S\right\}$ tem a propriedade $W($ forte $)$.

Observação 2.4 Todo espaço convexo tem a propriedade $W$.

prova: Seja $X$ um espaço convexo, e seja $p \in X$. Definimos $H: X \times I \longrightarrow X$ por:

$$
H(x, t)=t p+(1-t) x
$$

Então $H(x, t) \neq x$ se $x \neq p$ e $t>0$.

Observação 2.5 A propriedade $W$ é preservada por produtos arbitrários. 
prova: Seja $\mathcal{F}=\left\{X_{s}: s \in S\right\}$ uma família de espaços com a propriedade $W$ e seja $p \in \prod_{s \in S} X_{s}$. Para cada $s \in S$ existe uma deformação $H_{s}: X_{s} \times I \longrightarrow X_{s}$ tal que $H_{s}\left(x_{s}, t\right) \neq x_{s}$ se $x_{s} \neq p_{s}$ e $t>0$.

Definimos a funçào $H: \prod_{s \in S} X_{s} \times I \longrightarrow \prod_{s \in S} X_{s}$ da seguinte forma:

$$
(H(x, t))_{s}=H_{s}\left(x_{s}, t\right) \quad \forall s \in S
$$

Então $H(x, t) \neq x$ se $x \neq p$ e $t>0$.

Lema 2.6 (de Ward) Todo espaço X perfeitamente normal com a propriedade $W$ tem CIP.

\section{Demonstração:}

Seja $K$ um subconjunto fechado não vazio de $X$, e seja $p \in K$. Por hipótese existe uma deformação $H: X \times I \longrightarrow X$ tal que $H(x, t) \neq x$ se $x \neq p$ e $t>0$.

Como $X$ é perfeitamente normal, existe uma função contínua $g: X \rightarrow I$ tal que $g(x)=0 \Leftrightarrow x \in K^{\circ}$

Definimos uma função $f: X \longrightarrow X$ por:

$$
f(x)=H(x, g(x)) \quad \forall x \in X
$$

Temos que $f$ é contínua e que $f i x(f)=K$, pois

$$
K \subseteq\{x: f(x)=x\} \subseteq\{x: g(x)=0\} \cup\{p\} \subseteq K \cup\{p\}=K
$$

\section{Observações:}

(1) Da obsevação (2.3) e do Lema de Ward temos que o produto enumerável de espaços metrizáveis, onde pelo menos um dos espaços tem a propriedade $W($ forte $)$, tem CIP.

(2) Como todo espaço convexo tem a propriedade $W$, segue do lema que todo espaço métrico convexo tem CIP. Em particular o cubo de Hilbert tem CIP. 


\subsection{Exemplos em espaços não métricos}

Nesta seçào vamos mostrar que a reta longa $L \in L \times S^{1}$ tem CIP, emquanto que seus compactificados de Stone-Čech, $L^{*}$ e $L^{*} \times S^{1}$ não. Em particular vemos que a hipóteses do lema de Ward que diz que o espaço tem que ser perfeitamente normal é essencial, pois $S^{1}$ tem a propriedade $W$ porém $L^{*} \times S^{1}$ nào tem CIP.

Exemplo 2.7 : L tem CIP

\section{Demonstração:}

Seja $K$ um subconjunto fechado não vazio de $L$ com ínfimo $a$.

\section{caso 1}

se $K$ tem supremo, seja $b=\sup (K)$. Entào $[a, b]$ contem $K$ e $[a, b]$ tem CIP:

Seja $f:[a, b] \longrightarrow I$ homeomorfismo. Como $K$ é subconjunto fechado de $[a, b]$, segue que $f\left(K^{\circ}\right)$ é fechado em I. Logo existe uma função contínua $g: I \longrightarrow I$ tal que $f i x(g)=f\left(K^{*}\right)$. Temos então:

$$
[a, b] \stackrel{f}{\longrightarrow} I \stackrel{g}{\longrightarrow} I \stackrel{f^{-1}}{\longrightarrow}[a, b]
$$

$f^{-1} \circ g \circ f:[a, b] \longrightarrow[a, b]$ é uma função contínua e $f i x\left(f^{-1} \circ g \circ f\right)=K$. Então $[a, b]$ tem C'IP.

Também vemos que $[a, b]$ é um retrato de $L$ :

seja $h: L \longrightarrow L$ definida por:

$$
h(x)= \begin{cases}a & \text { se } x \leq a \\ b & \text { se } x \geq b \\ x & \text { se } x \in[a, b]\end{cases}
$$

Então $h(L)=[a, b]$ e $h$ é contínua. A função $j=f^{-1} \circ g \circ f:[a, b] \longrightarrow[a, b]$ é tal que $f i x(j)=K$. Seja $j \circ h: L \longrightarrow L$

$$
L \stackrel{h}{\longrightarrow}[a, b] \stackrel{j}{\longrightarrow}[a, b] \hookrightarrow L
$$

Temos que : 
1. $\forall x \in K, j \circ h(x)=x$.

2. Se $x \notin K$ então $x \in[a, b]-K$ ou $x \notin[a, b]$.

Caso $x \in[a, b]-K$ entào $h(x)=x$ mas $j(x) \neq x \quad$ e portanto $j \circ h(x) \neq x$.

Caso $x \notin[a, b]$ então $h(x)=a$ ou $h(x)=b$. Em qualquer caso $j \circ h(x) \neq x$.

De 1 e 2 segue que $f i x(j \circ h)=K$.

caso 2 :

Se $K$ é não limitado em $L$, então $L-K$ é a união de uma família disjunta de intervalos \{]$a_{\alpha}, b_{\alpha}[: \alpha \in \Lambda\}$.

Para cada $\alpha \in$ I. seja $f_{\alpha}$ um autohomeomorfismo de $\left[a_{\alpha}, b_{\alpha}\right]$ com fix $\left(f_{\alpha}\right)=\left\{a_{\alpha}, b_{\alpha}\right\}$.

Definimos a função $f: L \longrightarrow L$ por:

$$
f(x)= \begin{cases}a & \text { se } x \leq a \\ x & \text { se } x \in K \\ f_{\alpha}(x) & \text { se } x \in] a_{\alpha}, b_{\alpha}[\text { para algum } \alpha \in \Lambda\end{cases}
$$

A função $f$ é contínua em $L$ :

Seja $x \in L$. Se $x \in L-K$,então $x \in] a_{\alpha}, b_{\alpha}\left[\right.$ para algum $\alpha \in \Lambda$. Como $f_{\alpha}$ é contínua e $f=f_{\alpha}$ em $] a_{\alpha}, b_{\alpha}[$, f é contínua em $x$.

Se $x \in K$, seja $V=] c, d[$ uma vizinhança aberta de $x$. Pode acontecer

(i) $V \subset K$ e então $\left.f\right|_{V}=i d$ e $f$ é contínua em $x$, ou

(ii) $V \not \subset K$. Temos as seguintes possibilidades:

(1) $x \neq a_{\alpha} \forall \alpha \in \Lambda$. Neste caso, se existe $\alpha \in \Lambda$ tal que $x<a_{\alpha}<d$, seja $A=\left[x, a_{\alpha}[\right.$ senão seja $A=\left[x, d[\right.$. Então se $y \in A, f(y)=y$ ou $f(y) \in] a_{\beta}, b_{\beta}\left[\subset\left[x, a_{\alpha}[\subset[x, d[\right.\right.$. Logo $f(A) \subset[x, d[$.

(2) $x=a_{\alpha}$ para algum $\alpha \in \Lambda$. Se $b_{\alpha}<d$, seja $A=\left[x, b_{\alpha}\left[\right.\right.$. Senão como $f_{\alpha}$ é contínua e $f_{\alpha}\left(a_{\alpha}\right)=a_{\alpha}$, existe $e \in\left[a_{\alpha}, d\left[\right.\right.$ tal que $f_{\alpha}\left(\left[a_{\alpha}, \epsilon\right]\right) \subset\left[a_{\alpha}, d[\right.$, seja, então. $A=[x, e[$. Igual que no caso anterior temos $f(A) \subset[x, d[$. 
(3) $x \neq b_{i 3} \forall 3 \in \Lambda$. Se existe $3 \in \Lambda$ tal que $c<b_{\beta}<x$, seja $\left.\left.B=\right] b_{\beta}, x\right]$, senão seja $B=] c, x]$. Nos dois casos temos $f(B) \subset] c, x]$.

(4) $x=b_{\beta}$ para algum $\beta \in \Lambda$. Seja $k=\max \left\{c, a_{\beta}\right\}$, como $f_{\beta}$ é contínua e $f\left(b_{\beta}\right)=b_{\beta}$, existe $\left.\left.\epsilon \in\right] k, b_{3}\right]$ tal que $\left.\left.\left.\left.f_{\alpha}(] e, b_{\beta}\right]\right) \subset\right] c, b_{\beta}\right]$. Seja então $\left.\left.B=\right] e, x\right]$.

Em todos os casos $A \cup B$ é uma vizinhança aberta de $x$, e $f(A \cup B) \subset V$.

Falta provar que fix $(f)=K$. Observemos que $] \leftarrow, a[\subseteq L-K$ e que para cada $\alpha \in \Lambda$ temos que $\left\{a_{\alpha}, b_{\alpha}\right\} \subseteq K^{\prime}$ e $] a_{\alpha}, b_{\alpha}\left[\subseteq L-K^{\circ}\right.$.

Seja $x \in L$,

(i) Se $x \in K$, entào $f(x)=x$ por definição de $f$

(ii) Se $x \notin K$ quer dizer que $x<a$ ou $x \in] a_{\alpha}, b_{\alpha}[$ para algum $\alpha \in \Lambda$. Em ambos $\operatorname{casos} f(x) \neq x$.

Temos então que para todo subconjunto fechado $K \subseteq L$, existe uma função contínua $f: L \longrightarrow L$ tal que fir $(f)=K$. Logo $L$ tem CIP.

Exemplo 2.8: $L^{*}$ nào tem CIP.

\section{Demonstração:}

Vamos provar que o conjunto $K^{\prime}=\left\{0, \omega_{1}\right\}$ não é um conjunto de pontos fixos de $L^{*}$.

Suponhamos $f: L^{*} \longrightarrow L^{*}$ uma função contínua tal que $f i x(f)=K$.

Como $f\left(\omega_{1}\right)=\omega_{1}$ e $f$ é contínua temos que $f^{-1}(\{0\})$ é limitado em $\omega_{1}$. Seja $\alpha_{1} \in \omega_{1}$ tal que $f^{-1}(\{0\}) \subset\left[0, \alpha_{1}\left[\right.\right.$, temos que $f(\beta)>0 \quad \forall \beta \geq \alpha_{1}$.

Seja agora $\alpha_{2}>\alpha_{1} \in \omega_{1}$ tal que $f^{-1}\left(\left[0, \alpha_{1}[) \subset\left[0, \alpha_{2}\left[\right.\right.\right.\right.$, então $f(\beta) \geq \alpha_{1} \quad \forall \beta \geq \alpha_{2}$. Continuando com este procedimento, obtemos uma sequência $\left(\alpha_{n}\right)_{n \in \omega}$ tal que para cada $n f^{-1}\left(\left[0, \alpha_{n}[) \subset\left[0, \alpha_{n+1}[\right.\right.\right.$.

Seja $\alpha=\sup \left\{\alpha_{n}: n \in \omega\right\} \quad \alpha<\omega_{1}$ pois $c f\left(\omega_{1}\right)=\omega_{1}$.

afirmação $1: f^{-1}([0 . a[) \subset[0, \alpha[$

se não, seja $\beta \geq \alpha$ tal que $\beta \in f^{-1}([0, \alpha[)$. Então $f(\beta) \in[0, \alpha[$. 
Como $a=\sup \left\{\alpha_{n}: n \in \omega\right\}$ existe $n_{0}$ tal que $f(\beta)<\alpha_{n_{0}} \leq \alpha$ i.e. $f(\beta) \in\left[0, \alpha_{n_{0}}[\right.$. Logo $\beta<\alpha_{n_{0}+1} \leq \alpha$ o que é uma contradiçào.

Seja $\gamma_{1}=a$ $f\left(\left[0, \gamma_{1}[)\right.\right.$ é limitado em $L^{*}$ pois $c f\left(\omega_{1}\right)=\omega_{1}$ e $c f\left(\gamma_{1}\right)$ é enumerável para cada $\mu \leq \gamma_{1}$. Seja entào $\gamma_{2} \in \omega_{1}$ tal que $f\left(\left[0, \gamma_{1}[) \subset\left[0, \gamma_{2}\left[\right.\right.\right.\right.$. De igual forma $f\left(\left[0, \gamma_{2}[)\right.\right.$ é limitado em $L^{*}$. $\log$ o existe $\gamma_{3}>\gamma_{2}$ tal que $f\left(\left[0, \gamma_{2}[) \subset\left[0, \gamma_{3}[)\right.\right.\right.$. Seja $\left(\gamma_{n}\right)_{n \in \omega}$ uma sequência tal que $\gamma_{n}<\omega_{1}$ e $f\left(\left[0, \gamma_{n}[) \subset\left[0, \gamma_{n+1}[\forall n \in \omega\right.\right.\right.$.

Seja $\gamma=\sup \left\{\hat{i n}_{n}: n \in \omega\right\}$ temos que $\gamma<\omega_{1}$ pela cofinalidade de $\omega_{1}$.

afirmaçào $2: f([0, \gamma[) \subset[0, \gamma[$

se nào seja $\beta<\gamma$ tal que $f(\beta) \geq \gamma$. Como $\gamma=\sup \left\{\gamma_{n}: n \in \omega\right\}$, existe $n_{0}$ tal que $\beta<\gamma_{n_{0}}<\gamma \operatorname{logo} \beta \in\left[0, \gamma_{n_{0}}\right.$ [ e portanto $f(\beta) \in\left[0, \gamma_{n_{0}+1}[\quad\right.$ o que é uma contradição.

Das afirmações 1 e 2 temos que $f([\alpha, \gamma]) \subset[\alpha, \gamma]$.

Por ser $[\alpha, \gamma]$ homeomorfo ao intervalo real $[0,1]$ e pelo teorema do valor intermediário para funçòes reais temos que existe $x \in[\alpha, \gamma]$ tal que $f(x)=x$ contradizendo $f i x(f)=K$.

Exemplo $2.9: L^{*} \times S^{1}$ não tem $C I P$.

\section{Demonstração:}

Sejam $q \in S^{1}$ e $h: L^{*} \longrightarrow L^{*} \times S^{1}$ a imersão definida por $h(x)=(x, q) \quad \forall x \in L^{*}$ Seja $K^{*}=\left\{h(0), h\left(\omega_{1}\right)\right\}$ fechado em $L^{*} \times S^{1}$. Vamos provar que $K^{\prime}$ não é um conjunto de pontos fixos de $L^{*} \times S^{1}$. Suponhamos, por contradição que existe uma função contínua $f: L^{*} \times S^{1} \longrightarrow L^{*} \times S^{1}$ tal que $f i x(f)=K$.

A ideia é usar argumentos similares aos usados para provar que $L^{*}$ não tem CIP. Porém para isto é necessário fazer algumas transformações pois tais argumentos estão baseados na ordem de $L^{*}$.

Sejam $V_{1}, V_{2}, \ldots$ uma sequência decrescente de intervalos abertos em $S^{1}$ tal que $\bigcap_{i=1}^{\infty} V_{i}=\{q\}$ e seja $\pi: L^{*} \times S^{1} \longrightarrow S^{1}$ a projeção na segunda coordenada. 
Consideramos a função $\pi \circ f \circ h: L^{*} \longrightarrow S^{1}$.

Temos que:

$$
(\pi \circ f \circ h)^{-1}(q)=(\pi \circ f \circ h)^{-1}\left(\bigcap_{i=1}^{\infty} V_{i}\right)=\bigcap_{i=1}^{\infty}(\pi \circ f \circ h)^{-1}\left(V_{i}\right)
$$

Por outro lado temos também :

$$
\begin{aligned}
& *(\pi \circ f \circ h)^{-1}(q)=h^{-1} \circ f^{-1}\left(L^{*} \times\{q\}\right) \\
& * f\left(h\left(\omega_{1}\right)\right)=h\left(\omega_{1}\right)=\left(\omega_{1}, q\right) \in L^{*} \times\{q\}
\end{aligned}
$$

Portanto $\bigcap_{i=1}^{\infty}(\pi \circ f \circ h)^{-1}(q)$ pode se escrever como a interseção de uma família enumerável de vizinhanças abertas de $\omega_{1}$. Como $\left\{\omega_{1}\right\}$ nào é um $G_{\delta}$ em $L^{*}$, existe $\alpha \in L$ tal que $\left[\alpha, \omega_{1}\right] \subset(\pi \circ f \circ h)^{-1}(q)$.

Entào $f \circ h\left(\left[\alpha \cdot \omega_{1}\right]\right) \subset \pi^{-1}(q)=L^{*} \times\{q\} h\left(L^{*}\right)$.

Com a ordem de $L^{*}$ em $L^{*} \times\{q\}$ e argumentos similares aos usados no exemplo (2.8), achamos $p \in] a \cdot \omega_{1}\left[\right.$ tal que $f(h(p))=h(p)$. Esta contradição prova que $L^{*} \times S^{1}$ nào tem CIP.

Exemplo 2.10 : $L \times S^{1}$ tem CIP.

\section{Demonstração:}

Seja $K^{\circ} \subset L \times S^{1}$ um conjunto fechado não vazio.

caso 1: Ir compacto

Seja $\pi_{1}: L \times S^{1} \longrightarrow L$ a projeção na primeira coordenada. Como $\pi_{1}$ é contínua. $\pi_{1}\left(K^{\prime}\right)$ é um subconjunto compacto de $L$. Logo $\pi_{1}(K) \subset[a, b] \subset L$.

[a.b] $\times S^{1}$ é um retrato de $L \times S^{1}$ homeomorfo a $I \times S^{1}$. Pelo lema de Ward $I \times S^{1}$ tem CIP. então $[a, b] \times S^{1}$ tem CIP.

$K \subset[a, b] \times S^{1}$, portanto $K$ é um conjunto de pontos fixos de $L \times S^{1}$.

caso 2 : $K$ não é compacto

Seja $Y=\left\{y \in S^{1}:\{x:(x, y) \in K\}\right.$ é não limitado em $\left.L\right\}$ 
afirmaçào 1: Existe $\alpha_{0} \in \omega_{1}$ tal que se $x>\alpha_{0}$ e $(x, y) \in K$ entào $y \in Y$.

Prova:

Suponhamos por contradição que para cada $\alpha \in \omega_{1}$, existem elementos $x_{\alpha}>\alpha$ e $y_{\alpha} \notin Y$ tais que $\left(x_{\alpha}, y_{\alpha}\right) \in K$.

$O$ conjunto $\left\{y_{\alpha}: \alpha \in \omega_{1}\right\}$ é não enumerável. (pois senão, existe $y \in\left\{y_{\alpha}: \alpha \in \omega_{1}\right\}$ tal que $\left\{3 \in \omega_{1}: y=y_{\beta}\right\}$ é não enumerável e portanto não limitado em $\omega_{1}$. Então $\left\{x_{\beta}:\left(x_{\beta}, y\right) \in \Lambda^{*}\right\}$ seria não limitado em $L$ e nesse caso $\left.y \in Y\right)$

Logo, como $S^{1}$ é compacto e tem base enumerável, existe $q \in\left\{y_{\alpha}: \alpha \in \omega\right\}$ ponto de acumulaçào completo de $\left\{y_{\alpha}: \alpha \in \omega_{1}\right\}$.

Seja $\beta_{0} \in \omega_{1}$. Para cada $n \in \omega$, escolhemos $\beta_{n+1}>\beta_{n}$ tal que :

(i) $x_{\beta_{n+1}}>x_{\beta_{n}}$

(ii) $\left\|q-y_{3_{n+1}}\right\|<1 / n+1$ onde \|\| é a norma usual de $\mathbb{R}^{2}$.

Seja agora, $x=\sup \left\{x_{\beta_{n}}: n \in \omega\right\}$

$\left(x_{\beta_{n}}, y_{\beta_{n}}\right) \in K^{\cdot} \forall n \in \omega$ e $(x, q)$ é ponto de acumulaçào de $\left\{\left(x_{\beta_{n}}, y_{\beta_{n}}\right): n \in \omega\right\}$. Como $K$ é fechado, segue que $(x, q) \in K$.

$\beta_{0}$ é arbitrário e $x>\beta_{0}$, portanto $q \in Y$, contra a suposição.

afirmação 2: Existe um conjunto fechado não limitado $C \subseteq \omega_{1}-\alpha_{0}$ tal que para todo par $\alpha_{1}, \alpha_{2} \in C$ tem-se $\left\{y:\left(\alpha_{1}, y\right) \in K^{\prime}\right\}=\left\{y:\left(\alpha_{2}, y\right) \in K^{\prime}\right\}$

Prova:

Seja $D$ um subconjunto denso enumerável de $Y$. Para cada $y \in D$, o conjunto $C_{y}=\left\{\alpha \in \omega_{1}:(\alpha, y) \in K^{\prime}\right\}$ é fechado, e não limitado em $\omega_{1}$. Fechado pois $K$ é fechado e não limitado por definição de $Y$.

O conjunto $C=\left(\omega_{1}-\alpha_{0}\right) \bigcap\left(\bigcap\left\{C_{y}: y \in{ }^{\prime} D\right\}\right)$ é fechado e nào limitado em $\omega_{1}$ : $\omega_{1}-\alpha_{0}$ é fechado e não limitado em $\omega_{1}$,

$|D|=\omega, c f\left(\omega_{1}\right)=\omega_{1}$ e para cada $y \in D C_{y}$ é c.u.b em $\omega_{1}, \operatorname{logo} \bigcap\left\{C_{y}: y \in D\right\}$ é c.u.b em $\omega_{1}$.

Para cada $\alpha \in C$ e $y \in D,(\alpha, y) \in K$, então $D \subseteq\left\{y:(\alpha, y) \in K^{\prime}\right\}$. 
Se $\alpha \in C$ e $(a . y) \in K$. pela afirmação 1 temos que $y \in Y$ pois $\alpha>\alpha_{0}$. Portanto $\left\{y:(\alpha, y) \in K^{*}\right\} \subset Y$.

Temos então $D \subset\left\{y:(\alpha, y) \in K^{-}\right\} \subset Y$ e $\bar{D}=\bar{Y}$. Como $\left\{y:(\alpha, y) \in K^{-}\right\}$é fechado, segue que é o próprio $Y$.

Vamos considerar agora um subconjunto $\left\{c_{\alpha}: \alpha \in \omega_{1}\right\}$ de $C$, isomorfo a $\omega_{1}$.

Para cada $\alpha \in \omega_{1}$ existe um homeomorfismo $h_{\alpha}$ entre $L_{\alpha}=\left[c_{\alpha}, c_{\alpha+1}\right]$ e $I$. Seja $\rho_{\alpha}$ a métrica definida em $L_{\alpha}$ por:

$$
\rho_{\alpha}(x, y)=\left\|h_{\alpha}(x)-h_{\alpha}(y)\right\| \forall x, y \in L_{\alpha}
$$

Para cada $\alpha \in \omega_{1}$, consideramos a deformação $H_{\alpha}: L_{\alpha} \times I \longrightarrow L_{\alpha}$ definida por:

$$
H_{\alpha}(x . t)=h_{\alpha}^{-1}\left((1-t) h_{\alpha}(x)+t\left(h_{\alpha}(x)\right)^{2}\right) \quad \forall(x, t) \in L_{\alpha} \times I
$$

Observamos que para cada $t>0$, o conjunto dos pontos fixos de $H_{\alpha}(., t)$ é $\left\{c_{\alpha}, c_{\alpha+1}\right\}$ Seja $K_{0}=K^{\circ} \cap\left(\left[0, c_{0}\right] \times S^{1}\right)$ e seja $\rho$ uma métrica em $S^{1}$ limitada por 1 .

Definimos uma deformaçào $G: S^{1} \times I \longrightarrow S^{1}$ como segue:

$$
G\left(e^{i \theta}, t\right)=e^{i(\theta+t)} \quad \forall\left(e^{i \theta}, t\right) \in S^{1} \times I
$$

Definimos também as seguintes métricas:

(1) $d$ em $\left[0, c_{0}\right] \times S^{1}$ como $d\left(\left(x_{1}, y_{1}\right),\left(x_{2}, y_{2}\right)\right)=\min \left\{\rho^{\prime}\left(x_{1}, x_{2}\right)+\rho\left(y_{1}, y_{2}\right), 1\right\}$ onde $\rho^{\prime}(x, y)=\|h(x)-h(y)\|$ com $h:\left[0, c_{0}\right] \longrightarrow I$ um homeomorfismo.

(2) $d_{\alpha}, \operatorname{em}\left[c_{\alpha}, c_{\alpha+1}\right] \times S^{1} \operatorname{como} d_{\alpha}\left(\left(x_{1}, y_{1}\right),\left(x_{2}, y_{2}\right)\right)=\min \left\{\rho_{\alpha}\left(x_{1}, x_{2}\right)+\rho\left(y_{1}, y_{2}\right), 1\right\}$

Seja $f: L \times S^{1} \longrightarrow L \times S^{1}$ definida por :

$$
f(x, y)= \begin{cases}\left(x \cdot G\left(y, d\left((x, y), K_{0}\right)\right)\right) & \text { se }(x, y) \in\left[0, c_{0}\right] \times S^{1} \\ \left(H_{\alpha}\left(x, d_{\alpha}\left((x, y), K^{\prime}\right)\right), G\left(y, d\left(\left(c_{0}, y\right), K_{0}\right)\right)\right) & \text { se }(x, y) \in L_{\alpha} \times S^{1} \operatorname{com} \alpha \in \omega_{1}\end{cases}
$$

* A função $f$ é contínua pois $G, d, H_{\alpha}, d_{\alpha}$ sào funçòes contínuas. 
* fix $(f)=K$ :

$$
(x, y) \in K \Rightarrow\left\{\begin{array}{l}
(x, y) \in K_{0} \\
\text { ou } \\
(x, y) \in L_{\alpha} \times S^{1}\left(\operatorname{logo} x>\alpha_{0} \in y \in Y\right)
\end{array}\right.
$$

Seja $(x, y) \in\left[0, c_{0}\right] \times S^{1}$

$d\left((x, y), K_{0}\right)=0 \Leftrightarrow(x, y) \in \kappa_{0}, \quad \log 0 \quad G\left(y, d\left((x, y), K_{0}\right)\right)=y \Leftrightarrow(x, y) \in K_{0}$.

Seja agora $(x, y) \in L_{\alpha} \times S^{1}$ para algum $\alpha \in \omega_{1}$.

$$
H_{\alpha}\left(x, d_{\alpha}\left((x, y), K^{\prime}\right)\right)=x \Leftrightarrow\left\{\begin{array}{c}
(x, y) \in K \\
\text { ou } \\
x \in\left\{c_{\alpha}, c_{\alpha+1}\right\}
\end{array}\right.
$$

No caso em que $x \in\left\{c_{\alpha}, c_{\alpha+1}\right\}$ :

se $\left(c_{\alpha}, y\right) \notin K$ entào pela afirmação $2,\left(c_{0}, y\right) \notin K_{0}$ e portanto $G\left(y, d\left(\left(c_{0}, y\right), K_{0}\right) \neq y\right.$ se $\left(c_{\alpha}, y\right) \in K$, então $\left(c_{0}, y\right) \in K_{0}$.

No caso em que $(x, y) \in K$, como $x \in L_{\alpha}$ temos que $x>\alpha_{0}$. Logo $y \in Y$ e $\left(c_{0}, y\right) \in K_{0}$.

Olhando os exemplos precedentes podemos ser tentados a concluir que toda variedade não métrica tem CIP. Mas no exemplo que segue vemos que isso não é verdade e também que a CIP nào é preservada por produtos.

Exemplo 2.11 O produto $L \times L$ da reta longa $L$ não tem CIP.

\section{Demonstração:}

Seja $K=L \times\{0\} \cup\{0\} \times L$.

Vamos supor que $K$ é o conjunto dos pontos fixos de uma funçào contínua

$f: L \times L \longrightarrow L \times L$. Demonstraremos uma sequência de afirmações que nos levarão a uma contradição.

Sejam $f_{1}, f_{2}$ funções tais que $f(x, y)=\left(f_{1}(x, y), f_{2}(x, y)\right)$ e seja $\Delta=\{(x, x): x \in L\}$ 
afirmação 1: pelo menos uma das funções $f_{1}, f_{2}$ é limitada em $\Delta$

Suponhamos que nenhuma das funçòes $f_{1}, f_{2}$ é limitada em $\Delta$. Vamos demonstrar que $f$ tem ponto fixo fora de $K$.

Como cada $f_{i}$ é nào limitada em $\Delta$, é possivel obter uma sequência monótona estritamente crescente $\left\{x_{n}: n \in \omega\right\}$ tal que:

$$
\begin{aligned}
& \text { (i) } f_{2}\left(x_{2 n+2}, x_{2 n+2}\right)>x_{2 n+1}>f_{2}\left(x_{2 n}, x_{2 n}\right) \\
& \text { (ii) } x_{2 n+2}>f_{1}\left(x_{2 n+1}, x_{2 n+1}\right)>x_{2 n}
\end{aligned}
$$

Seja $p=\lim _{n \rightarrow \infty} x_{n}$. Entào $p=\lim _{n \rightarrow \infty} x_{2 n}$ e $p=\lim _{n \rightarrow \infty} x_{2 n+1}$.

Da construção da sequência $\left\{x_{n}\right\}$ temos que $p=\lim _{n \rightarrow \infty} f\left(x_{2 n+i}, x_{2 n+i}\right)$ para $i=1,2$. Logo pela continuidade da funçào $f$ temos que $f(p, p)=(p, p) \notin K$.

Sem perda de generalidade vamos supor que $f_{1}$ é limitada em $\Delta$ por $\beta \in \omega_{1}$. Para cada ordinal limite $\alpha \in \omega_{1}$, consideramos um ordinal $\phi(\alpha)<\alpha$ tal que $f_{1}(\alpha, \phi(\alpha))<\beta+1$. observamos que este $\phi(\alpha)$ existe pois $f_{1}(\alpha, \alpha)<\beta+1$ e $f_{1}$ é contínua.

Usando o Pressing Down lemma, obtemos um conjunto nào enumerável $A \subset \omega_{1}$ e um ordinal $\gamma \in \omega_{1}$ tais que para cada $\alpha \in A, \phi(\alpha)=\gamma$. Então $f_{1}(\alpha, \gamma)<\beta+1 \forall \alpha \in A$.

afirmação 2: o conjunto $\left\{x \in L: f_{1}(x, \gamma)>\beta+2\right\}$ é limitado.

Suponhamos que $\left\{x \in L: f_{1}(x, \gamma)>\beta+2\right\}$ é não limitado. Então é possível obter sequências $\left\{x_{n}\right\} \subset L$ e $\left\{\alpha_{n}\right\} \subset A$ tais que :

$$
\begin{aligned}
& x_{0}<\alpha_{0}<x_{1}<\alpha_{1}<x_{2}<\ldots<\alpha_{n}<x_{n+1}<\alpha_{n+1}<\ldots \\
& \text { para cada } n, f_{1}\left(x_{n}, \gamma\right)>\beta+2 \text { e } f_{1}\left(\alpha_{n}, \gamma\right)<\beta+1
\end{aligned}
$$

mas $\sup _{n \in \omega}\left\{x_{n}\right\}=\sup _{n \in \omega}\left\{\alpha_{n}\right\}$, então $f_{1}$ não seria contínua.

afirmação 3: a função $f_{1}$ é limitada $\mathrm{em} L \times\{\gamma\}$

Pela afirmação 2 , existe $a \in L$ tal que $f_{1}(x, \gamma) \leq \beta+2 \forall x \geq a$.

Como $[0, a] \times\{\gamma\}$ é compacto segue que $f_{1}$ é limitada em $L \times\{\gamma\}$.

Seja agora $b$ um elemento de $L$ tal que $b=\inf \left\{\gamma \in L: f_{1}\right.$ é limitado em $\left.L \times\{\gamma\}\right\}$. 
afirmação 4: a função $f_{1}$ é limitada $\mathrm{em} L \times\{b\}$

Seja $\left\{b_{n}: n \in \omega\right\} \subset\left\{\gamma \in L: f_{1}\right.$ e limitado em $\left.L \times\{\gamma\}\right\}$ uma sequência monótona decrescente convergindo para $b$. Para cada $n \in \omega$, seja $m_{n}$ um limite superior para $f_{1}$ em $L \times\left\{b_{n}\right\}$. Então $\sup _{n \in \omega}\left\{m_{n}\right\}$ é um limite superior para $f_{1}$ em $L \times\{b\}$.

Notemos que $b>0$ pois a função $f$ é a identidade em $L \times\{0\}$, portanto $f_{1}$ é nào limitada em $L \times\{0\}$.

afirmação j: a função $f_{1}$ é não limitada $\mathrm{em} L \times\{b\}$.

Sejam $\lambda \in \omega_{1}$ arbitrário e $\left\{b_{n}\right\}$ uma sequência monótona crescente convergendo para b. Para cada $n, f_{1}$ é não limitada em $L \times\left\{b_{n}\right\}$.

$\mathrm{O}$ conjunto $\left\{x \in L: f_{1}\left(x, b_{n}\right) \geq \lambda\right\}$ é fechado e não limitado em $L$. Fechado pois $\left\{x \in L: f_{1}\left(x, b_{n}\right)<\lambda\right\}$ é aberto em $L$ pela continuidade de $f_{1}$. Nào limitado porque senão, existiria $x_{0} \in L$ tal que $\forall x>x_{0}, f_{1}\left(x, b_{n}\right)<\lambda$. E como $\left[0, x_{0}\right] \times\left\{b_{n}\right\}$ é compacto. teriamos $f_{1}$ limitada em $L \times\left\{b_{n}\right\}$.

Portanto pela continuidade de $f_{1}$, o conjunto $\left\{\alpha \in \omega_{1}: f_{1}\left(\alpha, b_{n}\right) \geq \lambda\right\}$ é um subconjunto fechado e nào limitado de $L$ i.e. $\left\{\alpha \in \omega_{1}: f_{1}\left(\alpha, b_{n}\right) \geq \lambda\right\}$ é c.u.b em $\omega_{1}$. Logo $\bigcap_{n \in \omega_{0}}\left\{\alpha \in \omega_{1}: f_{1}\left(\alpha, b_{n}\right) \geq \lambda\right\}$ é um subconjunto fechado e não limitado de $\omega_{1}$. De novo pela continuidade de $f_{1}$ temos que para cada $\alpha$ nesse conjunto $f_{1}(\alpha, b) \geq \lambda$. Como $\lambda$ é arbitrário segue que $f_{1}$ é nào limitada em $L \times\{b\}$.

\subsection{Condições suficientes para espaços produto}

Embora as propriedades $W$ e $W($ forte $)$ são preservadas por produtos arbitrários, não acontece o mesmo para o produto de espaços perfeitamente normais. Consequentemente o lema de Ward nào pode ser usado para determinar se tal produto tem CIP. O seguinte teorema apresenta condições suficientes para que um produto infinito tenha CIP. 
Teorema 2.12 Seja $\lambda$ um cardinal infinito. Para cada $a \in \lambda$, seja $Y_{\alpha}$ um espaço topológico com base $\{B(\alpha . \gamma): \gamma \in \lambda\}$ (i.e $\left.w\left(Y_{\alpha}\right) \leq \lambda\right)$.

Suponha que para cada par de subconjuntos finitos $F$ e $G$ de $\lambda$ tais que $F=\left\{\alpha_{1}, \ldots, \alpha_{n}\right\}$, $G=\left\{\gamma_{1}, \ldots, \gamma_{n}\right\}$ eada $3 \in \lambda$ não nulo, existe uma função contínua

$$
h: Y_{\alpha_{1}} \times \ldots \times Y_{\alpha_{n}} \times Y_{\beta} \longrightarrow Y_{\beta}
$$

tal que para $x \in Y_{\alpha_{1}} \times \ldots \times Y_{\alpha_{n}} \times Y_{\beta}, h(x)=\pi_{\{\beta\}}(x)$ se e somente se $\pi_{F}(x) \notin B\left(\alpha_{1}, \gamma_{1}\right) \times \ldots \times B\left(\alpha_{n}, \gamma_{n}\right)$. Então $\prod\left\{Y_{\alpha}: \alpha \in \lambda\right\}$ tem CIP.

\section{Demonstração:}

Seja $\left\{\left(F_{3} \cdot\left(i_{3}\right): \beta \in \lambda\right\}\right.$ uma enumeração do conjunto :

$$
\{(F, G): F \text { e } G \text { são subconjuntos finitos de } \lambda\}
$$

tal que $F_{\beta} \subset \beta \quad \forall \beta \in \lambda$.

Seja $K$ um subconjunto fechado de $Y=\prod\left\{Y_{\alpha}: \alpha \in \lambda\right\}$. Vamos construir uma função contínua $f: Y \rightarrow Y$ tal que $f i x(f)=K$. A construção será por limite inverso.

Para cada $3 \operatorname{com} 1 \leq \beta \leq \lambda, f_{\beta}: \prod\left\{Y_{\alpha}: \alpha \in \beta\right\} \longrightarrow \prod\left\{Y_{\alpha}: \alpha \in \beta\right\}$ é tal que:

$$
\begin{array}{ll}
\text { (i) } \pi_{\mu}^{\beta} f_{3}(x)=f_{\mu} \pi_{\mu}^{\beta}(x) & \forall \mu<\beta, \forall x \in \prod\left\{Y_{\alpha}: \alpha \in \beta\right\} \\
\text { (ii) } f_{\beta}(x)=x & \forall x \in \pi_{\beta}(K)
\end{array}
$$

onde $\pi_{\mu}^{\beta}$ é a restriçào dos elementos de $\prod\left\{Y_{\alpha}: \alpha \in \beta\right\}$ a $\prod\left\{Y_{\alpha}: \alpha \in \mu\right\}$, e $\pi_{\beta}$ é a restriçào dos elementos de $Y$ a $\prod\left\{Y_{\alpha}: \alpha \in \beta\right\}$.

- No nível 1 , seja $f_{1}: Y_{0} \longrightarrow Y_{0}$ a identidade.

- No nível $\delta \leq \lambda \operatorname{com} \delta$ ordinal limite, supomos que $f_{\beta}$ é uma função contínua satisfazendo (i) e (ii) para todo $\beta<\delta$.

Para cada $x \in \prod\left\{Y_{\alpha}: \alpha<\delta\right\}$, definimos $f_{\delta}$ tal que :

$$
\text { (vi) } \pi_{\{\beta\}}^{\delta} f_{\mathcal{S}}(x)=\pi_{\{\beta\}}^{\beta+1} f_{\beta+1} \pi_{\beta+1}^{\delta}(x)
$$

Então $\pi_{3}^{\delta} f_{\mathcal{\delta}}(x)=\left(\pi_{\{\alpha\}}^{\alpha+1} f_{\mathcal{\delta}}(x)\right)_{\alpha<\beta}=\left(\pi_{\{\alpha\}}^{\alpha+1} f_{\alpha+1} \pi_{\alpha+1}^{\delta}(x)\right)_{\alpha<\beta}=f_{\beta} \pi_{\beta}^{\delta}(x) \quad$ e $\quad$ (i) se verifica. 
Se $x \in \pi_{\delta}\left(K^{\circ}\right)$ entào $f_{\mathcal{S}}(x)=x$ :

$x \in \pi_{\delta}(K) \Rightarrow \pi_{3}^{\delta}(x) \in \pi_{3}(K) \forall \beta<\delta \Rightarrow f_{\beta} \pi_{\beta}^{\delta}(x)=\pi_{\beta}^{\delta}(x) \forall \beta<\delta \Rightarrow f_{\delta}(x)=x$

- No nivel $\beta+1$ supomos $f_{\beta}$ construida satisfazendo (i) e (ii). Temos dois casos a considerar:

caso 1:

$F_{\beta}=\left\{\alpha_{1}, \ldots, \alpha_{n}\right\}, G_{i 3}=\left\{\gamma_{1}, \ldots, \gamma_{n}\right\}$ e $B\left(\alpha_{1}, \gamma_{1}\right) \times \ldots \times B\left(\alpha_{n}, \gamma_{n}\right) \cap \pi_{F_{\beta}}(K) \neq \emptyset$. Definimos : $\pi_{3}^{3+1} f_{\beta+1}(x)=f_{\beta} \pi_{\beta}^{\beta+1}(x) \quad \mathrm{e}$

$$
\text { (v) } \pi_{\{\beta\}}^{\beta+1} f_{\beta+1}(x)=\pi_{\{\beta\}}^{\beta+1}(x)
$$

caso 2:

$F_{\beta}=\left\{\alpha_{1}, \ldots \alpha_{n}\right\}, G_{3}=\left\{\gamma_{1}, \ldots, \gamma_{n}\right\}$ e $B\left(\alpha_{1}, \gamma_{1}\right) \times \ldots \times B\left(\alpha_{n}, \gamma_{n}\right) \cap \pi_{F_{\beta}}(K)=\emptyset$.

Podemos usar entào a funçào $h: Y_{\alpha_{1}} \times \ldots \times Y_{\alpha_{n}} \times Y_{\beta} \longrightarrow Y_{\beta}$ da hipótese e definimos $f_{\beta+1}$ como segue : se $x \in \prod\left\{Y_{\alpha}: \alpha<\beta+1\right\}$

$$
\begin{aligned}
& \text { (iii) } \pi_{\beta}^{\beta+1} f_{\beta+1}(x)=f_{\beta} \pi_{\beta}^{\beta+1}(x) \\
& \text { (iv) } \pi_{\{\beta\}}^{\beta+1} f_{\beta+1}(x)=h \circ \pi_{F_{\beta} \cup\{\beta\}}^{\beta+1}(x)
\end{aligned}
$$

Então, se $x \in \pi_{3+1}\left(K^{\circ}\right)$ temos que :

$$
\left.\begin{array}{r}
\pi_{\beta}^{3+1}(x) \in \pi_{\beta}(K) \\
\pi_{3}^{3+1} f_{3+1}(x)=f_{\beta} \pi_{\beta}^{\beta+1}(x)
\end{array}\right\} \Rightarrow \pi_{\beta}^{\beta+1} f_{\beta+1}(x)=\pi_{3}^{\beta+1}(x) ;
$$

como $F_{\beta} \subset \beta, \pi_{F_{3}}^{3+1}(x) \in \pi_{F_{\beta}}\left(K^{\prime}\right)$, entào $\pi_{F_{\beta}}^{\beta+1}(x) \notin B\left(\alpha_{1}, \gamma_{1}\right) \times \ldots \times B\left(\alpha_{n}, \gamma_{n}\right) \quad$ e

$$
h \circ \pi_{F_{3} \cup\{\beta\}}^{3+1}(x)=\pi_{\{\beta\}}^{\beta+1}(x)
$$

De (1) e (2) temos : $\pi_{3+1}(x) \in \Lambda \Rightarrow f_{\beta+1}(x)=x$.

Seja $f=f_{\lambda}$

$f: \prod\left\{Y_{\alpha}: \alpha<\lambda\right\} \longrightarrow \prod\left\{Y_{o}: \alpha<\lambda\right\}$ é uma funçào contínua tal que $f(x)=x \forall x \in K$ (por construçào).

Só resta provar que se $y \notin K$ então $f(y) \neq y$. 
Se $y \notin K$ existe uma vizinhança de $y$, aberto básico, disjunta de $K$. Portanto existem conjuntos $F=\left\{\alpha_{1}, \ldots, \alpha_{n}\right\}$ e $G=\left\{\gamma_{1}, \ldots, \gamma_{n}\right\}$ com $F, G \subset \lambda$ tais que

$$
\pi_{F}(y) \in B\left(\alpha_{1}, \gamma_{1}\right) \times \ldots \times B\left(\alpha_{n}, \gamma_{n}\right) \subset \pi_{F}(Y-K)
$$

Seja $\beta \in \lambda$ tal que $F=F_{\beta}$ e $G=G_{\beta}^{\prime}$, entào $\pi_{\{\beta\}}^{\beta+1} f_{\beta+1} \pi_{\beta+1}(y)=h \circ \pi_{F_{\beta} \cup\{\beta\}}(y)$ onde $h$ é como em (iv). Logo $h \circ \pi_{F_{\beta} \cup\{\beta\}}(y) \neq \pi_{\{\beta\}}(y)$, mas $\pi_{\{\beta\}}^{\beta+1} f_{\beta+1} \pi_{\beta+1}(y)=$ $\pi_{\{\beta\}}^{\beta+1} \pi_{\beta+1} f(y)=\pi_{\{\beta\}} f(y)$.

Portanto $\pi_{\{\beta\}} f(y) \neq \pi_{\{\beta\}}(y)$ e consequentemente $f(y) \neq y$.

Lema 2.13 Seja $\lambda$ um cardinal infinito. Suponhamos que para cada $\alpha \in \lambda, Y_{\alpha} \dot{e}$ um espaço localmente metrizável de peso menor ou igual a $\lambda$ e com a propriedade $W$ (forte). Então $\left\{Y_{\alpha}: \alpha \in \lambda\right\}$ satisfaz as hipóteses do teorema (2.12).

\section{Demonstração:}

$Y_{\alpha}$ é localmente metrizável, logo é regular.

Para cada $x \in Y_{\alpha}$, existe $U$ uma vizinhança aberta de $x$ tal que $U$ é metrizável. Como $\{B(\alpha, \gamma): \gamma<\lambda\}$ é base para $Y_{\alpha}$, existe $\gamma_{0}<\lambda$ tal que $x \in B\left(\alpha, \gamma_{0}\right) \subset \overline{B\left(\alpha, \gamma_{0}\right)} \subset U$. Podemos então supor, sem perda de generalidade que $\overline{B(\alpha, \gamma)}$ é metrizável $\forall \alpha, \gamma<\lambda$. Para cada par $(F, G)$ de subconjuntos finitos de $\lambda$ tais que $F=\left\{\alpha_{1}, \ldots, \alpha_{n}\right\}$ e $G=\left\{\gamma_{1}, \ldots, \gamma_{n}\right\}$, e cada $0<\beta<\lambda$ seja a função $d: Y_{\alpha_{1}} \times \ldots \times Y_{\alpha_{n}} \times Y_{\beta} \longrightarrow \mathbb{R}$ definida por:

$$
d(x)=\left\{\begin{array}{l}
\operatorname{dist}\left(\pi_{F}(x), Y_{\alpha_{1}} \times \ldots \times Y_{\alpha_{n}}-B\left(\alpha_{1}, \gamma_{1}\right) \times \ldots \times B\left(\alpha_{n}, \gamma_{n}\right)\right) \\
\quad \text { se } \pi_{F}(x) \in B\left(\alpha_{1}, \gamma_{1}\right) \times \ldots \times B\left(\alpha_{n}, \gamma_{n}\right) \\
0 \quad \text { caso contrário }
\end{array}\right.
$$

Definimos então a função $h: Y_{\alpha_{1}} \times \ldots \times Y_{\alpha_{n}} \times Y_{\beta} \longrightarrow Y_{\beta}$ por:

$$
h(x)=H_{\beta}\left(\pi_{\{\beta\}}(x), \frac{d(x)}{1+d(x)}\right)
$$


onde $H_{\beta}: Y_{\beta} \times I \longrightarrow Y_{\beta}$ é uma deformaçào tal que $H_{\beta}(y, t)=y \Leftrightarrow t=0$.

Temos que:

$$
\begin{gathered}
h(x)=\pi_{\{\beta\}}(x) \Leftrightarrow H_{\beta}\left(\pi_{\{\beta\}}(x), \frac{d(x)}{1+d(x)}\right)=\pi_{\{\beta\}}(x) \Leftrightarrow \\
\Leftrightarrow d(x)=0 \Leftrightarrow \pi_{F}(x) \notin B\left(\alpha_{1}, \gamma_{1}\right) \times \ldots \times B\left(\alpha_{n}, \gamma_{n}\right)
\end{gathered}
$$

como queríamos.

\section{Observações :}

- As variedades não métricas com a propriedade $W$ (forte) satisfazem o lema $(2.13)$.

- Embora foi bastante trabalhoso provar que $L \times S^{1}$ tem CIP, segue do teorema (2.12) e do lema (2.13) que $\left(L \times S^{1}\right)^{\kappa}$ tem CIP para todo cardinal não enumerável $\kappa$.

Teorema 2.14 Sejam $\lambda$ um cardinal infinito $e\left\{Y_{\alpha}: \alpha \in \lambda\right\}$ uma família não vazia de espaços métricos separáveis com a propriedade $W$ (forte). Então o espaço produto $\prod\left\{Y_{\alpha}: \alpha \in \lambda\right\}$ tem CIP.

\section{Demonstração:}

Para cada $\alpha<\lambda, Y_{\alpha}$ é um espaço métrico separável, portanto $Y_{\alpha}$ tem uma base enumerável (i.e. $w\left(Y_{\alpha}\right)=\omega \leq \lambda \forall \alpha<\lambda$ ).

Pelo lema (2.13) a família $\left\{Y_{\alpha}: \alpha \in \lambda\right\}$ satisfaz as hipóteses do teorema (2.12), então $\prod\left\{Y_{\alpha}: \alpha \in \lambda\right\}$ tem CIP.

\section{Observação :}

- $\mathbb{R}^{\kappa}$ e $\left(S^{1}\right)^{\kappa}$ tem CIP para todo cardinal $\kappa$ : pelo lema de Ward se $\kappa$ é enumerável e por teorema (2.14) se $\kappa$ é não enumerável. 
Lema 2.15 Seja $\lambda$ um cardinal infinito. Suponha que para cada $\alpha \in \lambda$, $Y_{\alpha}$ é um espaço zero-dimensional não trivial com $w\left(Y_{\alpha}\right) \leq \lambda$. Então $\left\{Y_{\alpha}: \alpha \in \lambda\right\}$ satisfaz as hipótesis do teorema (2.12) i.e. $\prod\left\{Y_{\alpha}: \alpha \in \lambda\right\}$ tem CIP.

\section{Demonstração:}

Para cada $\alpha \in \lambda$, seja $\{B(\alpha, \gamma): \gamma \in \lambda\}$ uma base de subconjuntos abertos e fechados para $Y_{\alpha}$. Sejam $F=\left\{\alpha_{1}, \ldots, \alpha_{n}\right\}$ e $G=\left\{\gamma_{1}, \ldots, \gamma_{n}\right\}$ subconjuntos finitos de $\lambda$, e seja $\beta \in \lambda, \beta \neq 0$.

Definimos $h: Y_{\alpha_{1}} \times \ldots \times Y_{\alpha_{n}} \times Y_{\beta} \longrightarrow Y_{\beta}$ como segue:

sejam $U$ um subconjunto aberto e fechado em $Y_{\beta}$ e $a, b$ dois pontos de $Y_{\beta}$ tais que $a \in U$ e b $\notin U$. Entào

$$
h\left(\pi_{F \cup\{\beta\}}(x)\right)= \begin{cases}\pi_{\{\beta\}}(x) & \text { se } \pi_{F}(x) \notin B\left(\alpha_{1}, \gamma_{1}\right) \times \ldots \times B\left(\alpha_{n}, \gamma_{n}\right) \\ a & \text { se } \pi_{F}(x) \in B\left(\alpha_{1}, \gamma_{1}\right) \times \ldots \times B\left(\alpha_{n}, \gamma_{n}\right) \text { e } \pi_{\{\beta\}}(x) \notin U \\ b & \text { se } \pi_{F}(x) \in B\left(\alpha_{1}, \gamma_{1}\right) \times \ldots \times B\left(\alpha_{n}, \gamma_{n}\right) \text { e } \pi_{\{\beta\}}(x) \in U\end{cases}
$$

A função $h$ assim definida é contínua:

vamos provar que a imagem inversa por $h$ de todo fechado de $Y_{\beta}$ é um fechado de $Y_{\alpha_{1}} \times \ldots \times Y_{\alpha_{n}} \times Y_{3}$

(1) $h^{-1}(a)=\left(B\left(\alpha_{1}, \gamma_{1}\right) \times \ldots \times B\left(\alpha_{n}, \gamma_{n}\right) \times Y_{\beta}-U\right) \cup\left(Y_{\alpha_{1}} \times \ldots \times Y_{\alpha_{n}} \times Y_{\beta}-B\left(\alpha_{1}, \gamma_{1}\right) \times\right.$ $\left.\left.\ldots \times B\left(\alpha_{n}, \gamma_{n}\right)\right) \times\{a\}\right)$.

$h^{-1}(a)$ é fechado em $Y_{\alpha_{1}} \times \ldots \times Y_{\alpha_{n}} \times Y_{\beta}$ pois $B\left(\alpha_{i}, \gamma_{i}\right)$ é aberto e fechado em $Y_{\alpha_{i}} \forall i=$ $1, \ldots, n$ e $\{a\}$ é fechado em $Y_{\beta}$.

(2) De igual forma que em (1) mostramos que $h^{-1}(b)$ é um subconjunto fechado de $Y_{\alpha_{1}} \times \ldots \times Y_{\alpha_{n}} \times Y_{\beta}$

(3) Seja $A$ um subconjunto fechado de $Y_{\beta}$. Temos vários casos a considerar.

* Se $a \notin A$ e $b \notin A$, entào

$h^{-1}(A)=\left(Y_{\alpha_{1}} \times \ldots \times Y_{\alpha_{n}} \times Y_{\beta}-B\left(\alpha_{1}, \gamma_{1}\right) \times \ldots \times B\left(\alpha_{n}, \gamma_{n}\right)\right) \times A$ subconjunto fechado de $Y_{\alpha_{1}} \times \ldots \times Y_{\alpha_{n}} \times Y_{\beta}$. 
* Se $a \in A \operatorname{com} A-\{a\} \neq \emptyset$ e $b \notin A$, então

$h^{-1}(A)=\left(\left(Y_{\alpha_{1}} \times \ldots \times Y_{\alpha_{n}} \times Y_{\beta}-B\left(\alpha_{1}, \gamma_{1}\right) \times \ldots \times B\left(\alpha_{n}, \gamma_{n}\right)\right) \times A\right) \cup\left(B\left(\alpha_{1}, \gamma_{1}\right) \times\right.$ $\left.\ldots \times B\left(\alpha_{n}, \gamma_{n}\right) \times\left(Y_{3}-l^{*}\right)\right)$ subconjunto fechado de $Y_{\alpha_{1}} \times \ldots \times Y_{\alpha_{n}} \times Y_{\beta}$

Os outros casos são análogos ao último acima.

Seja $x \in Y_{\alpha_{1}} \times \ldots \times Y_{i_{n}} \times Y_{\beta}$.

Temos que $h(x)=\pi_{\{\beta\}}(x) \Leftrightarrow \pi_{F}(x) \notin B\left(\alpha_{1}, \gamma_{1}\right) \times \ldots \times B\left(\alpha_{n}, \gamma_{n}\right)$.

Corolário 2.16 Sejam $\lambda$ um cardinal infinito $e\left\{Y_{\alpha}: \alpha \in \lambda\right\}$ uma família não vazia de espaços discretos. cada um dos quais tem no máximo uma quantidade enumerável de elementos. Entrio $\prod\left\{Y_{;}: \alpha \in \lambda\right\}$ tem CIP.

Teorema 2.17 Seja M um espaço métrico conexo com a propriedade $W($ forte) $e$ seja $Y$ um espaco normal totalmente desconexo. Então $M \times Y$ tem CIP se e somente se $Y$ é perfeitamente normal.

\section{Demonstração:}

$(\Leftarrow)$ seja $Y$ um espaço perfeitamente normal. Como $M$ é métrico, $M \times Y$ é perfeitamente normal e como $M$ tem a propriedade $W$ (forte) $M \times Y$ também tem a propriedade $W(f$ ort $\epsilon)$. Entào pelo lema de Ward $M \times Y$ tem CIP.

$(\Rightarrow)$ Suponhamos que.$/ \times Y$ tem CIP e que $Y$ nào é perfeitamente normal. Como $Y$ é normal, existe um subconjunto fechado $K$ de $Y$ que nào é um $G_{\delta}$.

Seja $p \in M$ e seja $A=\{(x, y) \in M \times Y: x=p$ ou $y \in K\}$

$A=\{p\} \times Y \cup M \times K$, então $A$ é fechado. Como $M \times Y$ tem CIP, existe uma função contínua $f: M \times Y \longrightarrow . M \times Y$ tal que $A=f i x(f)$.

$M$ é conexo portanto $M \times\{y\}$ e $f(M \times\{y\})$ são conexos $\forall y \in Y$

Como $Y$ é totalmente desconexo, a componente conexa de $(p, y)$ é $M \times\{y\}$ para cada $y \in Y . f(p, y)=(p, y), \operatorname{logo}(p, y) \in f(M \times\{y\})$ que é conexo. Então $f(M \times\{y\}) \subset M \times\{y\}$. 
Escolhemos um ponto $q \neq p$ em $M$. Seja $\delta=\rho(p, q)$ onde $\rho$ denota a distância em $M$ e seja $f_{1}=\pi \circ f$ onde $\pi: M \times Y \longrightarrow M$ denota a projeção na primeira coordenada.

Para cada $n \in \omega$ definimos $U_{n}=\left\{y \in Y: \rho\left(q, f_{1}(q, y)\right)>\delta / n\right\}$

afirmação: $Y-K=\bigcup_{n=1}^{\infty} U_{n}$

Primeiro seja $y \in Y-K$.

Como $q \neq p$ temos que $f(q, y) \neq(q, y)$, mas $f(q, y) \in M \times\{y\}$ então $f_{1}(q, y) \neq q$. Segue que $\rho\left(q, f_{1}(q, y)\right)>0$ e existe $n>0$ tal que $\rho\left(q, f_{1}(q, y)\right)>\delta / n$. Logo $y \in U_{n}$.

Seja agora $y \in \bigcup_{n=1}^{\infty} U_{n}$ entào existe $n_{0} \in \omega$ tal que $\rho\left(q, f_{1}(q, y)\right)>\delta / n_{0}$. Portanto $q \neq \pi \circ f(q, y)$ e $y \notin K$.

$\mathrm{K}$ nào é um $G_{\delta}^{\prime} \Rightarrow Y-K$ não é um $F_{\sigma} \Rightarrow Y-K \neq \bigcup_{n=1}^{\infty} \overline{U_{n}}$.

Quer dizer que para algum $n \in \omega$, existe $z \in K \cap \overline{U_{n}}$. Logo $f(q, z)=(q, z)$ e $f_{1}(q, z)=q$. No entanto temos que $\rho\left(q, f_{1}(q, y)\right) \geq \delta / n \quad \forall y \in \overline{U_{n}}$.

Supondo $Y$ não perfeitamente normal chegamos a uma contradição, então $Y$ deve ser perfeitamente normal.

No resultado que segue mostramos que o produto cartesiano de grupos compactos não preserva a CIP. Concretamente vamos ver que se $\lambda$ é um cardinal não enumerável $S^{1} \times\left(Z_{2}\right)^{\wedge}$ não tem CIP, porém $S^{1}$ e $\left(Z_{2}\right)^{\wedge}$ são grupos compactos com CIP.

Teorema 2.18 Seja $\lambda$ um cardinal não enumerável e seja $\left(Z_{2}\right)^{\lambda}$ o produto de $\lambda$ cópias do espaço discreto $Z_{2}=\{0,1\}$. Então $S^{1} \times\left(Z_{2}\right)^{\lambda}$ não tem CIP.

\section{Demonstração:}

$S^{1}$ é um espaço métrico conexo. $\left(Z_{2}\right)^{\wedge}$ é um espaço compacto Hausdorff e portanto normal. Se provarmos que $\left(Z_{2}\right)^{\lambda}$ é totalmente desconexo e não é perfeitamente normal, entào o teorema fica provado aplicando o teorema (2.17)

afirmação 1: $\left(Z_{2}\right)^{\lambda}$ é totalmente desconexo.

Suponhamos que não seja, então existem dois pontos $p, q \in\left(Z_{2}\right)^{\wedge}$ tais que a quasi- 
componente $\mathcal{C}_{p}$ de $p$ coincide com a quase-componente $\mathcal{C}_{q}$ de $q$.

$p \neq q \Rightarrow \exists \alpha<\lambda \exists i \in\{0,1\}$ tal que se $\pi_{\{\alpha\}}(p)=i$ então $\pi_{\{\alpha\}}(q)=1-i$.

Seja $U_{p}=\prod_{\beta<\alpha} Z_{2} \times\{i\} \times \prod_{\alpha<\beta<\lambda} Z_{2}$. $U_{p}$ é vizinhança aberta e fechada de $p$, logo $\mathcal{C}_{p} \subset U_{p}$. Mas $q \notin U_{p}$, entào $\mathcal{C}_{p} \neq \mathcal{C}_{q}$.

afirmação 2: $\left(Z_{2}\right)^{\wedge}$ não é perfeitamente normal.

Seja $x \in\left(Z_{2}\right)^{\lambda} .\{x\}$ é um fechado pois $\left(Z_{2}\right)^{\wedge}$ é Hausdorff. Suponhamos que $\left(Z_{2}\right)^{\lambda}$ é perfeitamente normal. então existe uma família enumerável de abertos básicos $\left\{I_{n}: n \in \omega\right\}$ tal que $\bigcap\left\{V_{n}: n \in \omega\right\}=\{x\}$.

Para cada $n$. seja $F_{n} \subset \lambda$. finito, tal que $V_{n}=\prod_{\alpha \in F_{n}}\left\{\pi_{\alpha}(x)\right\} \times \prod_{\lambda-F_{n}} Z_{2}$.

$\bigcup_{n=1}^{\infty} F_{n}$ é um conjunto enumerável, logo existe $\alpha \in\left(\lambda-\bigcup_{n=1}^{\infty} F_{n}\right)$. Temos que $\pi_{\{\alpha\}} V_{n}=Z_{2} \quad \forall n \in \omega$, então $\bigcap_{n=1}^{\infty} \pi_{\{\alpha\}} V_{n}=Z_{2}$ e $\bigcap_{n=1}^{\infty} V_{n} \neq\{x\}$.

No seguinte teorema usamos o conceito de CIP para dar uma caracterização dos espaços compactos zero-dimensionais que são metrizáveis.

Teorema 2.19 Seja $M$ um espaço métrico conexo com a propriedade $W$ (forte) e seja X um espaço compacto zero-dimensional. Então $M \times X \times X$ tem $C I P$ se e somente se $\mathrm{X}$ é metrizável.

\section{Demonstração:}

$(\Leftarrow)$ Se $X^{\prime}$ é metrizável, então $M \times X \times X$ é também metrizável. Como $M \times X \times X^{r}$ tem a propriedade $W$ (forte), pelo lema de Ward tem CIP.

$(\Rightarrow)$ Suponhamos que $M \times X \times X$ tem CIP. Como $X \times X$ é um espaço compacto zero-dimensional. então $X \times X$ é normal e totalmente desconexo.

Pelo teorema (2.17), segue que $X \times X$ é perfeitamente normal. A diagonal $\Delta$ de $X \times X$ é fechada, $\log o \Delta$ é um $G_{\delta}$ em $X \times X$ e portanto $X$ é metrizável. 
Para mostrar que o compactificado de Stone-Cech $\beta \mathbb{N}$ dos inteiros positivos $\mathbb{N}$ não tem CIP, provamos o seguinte lema preliminar.

Lema 2.20 Seja $f: \mathbb{N} \longrightarrow \mathbb{N}$ uma função contínua definida no conjunto dos inteiros positivos $\mathbb{N}$. Entĩo uma das seguintes afirmações é verdadeira:

(i) existe um subconjunto infinito $A$ de $\mathbb{N}$ tal que para todo $x \in A, f(x)=x$.

(ii) existe um subconjunto infinito $A$ de $\mathbb{N}$ tal que para todo $x \in A, f(x) \notin A$.

\section{Demonstração:}

Temos dois casos a considerar.

caso 1: suponhamos que existe $y \in \mathbb{N}$ tal que $f^{-1}(y)$ é infinito. Então

$$
A=f^{-1}(y)-\{y\}
$$

satisfaz (ii).

caso 2: suponhamos que para cada $y \in \mathbb{N}, f^{-1}(y)$ é finito. Seja $x_{0} \in \mathbb{N}$ e, para cada $n \geq 1$, seja $x_{n}$ um inteiro positivo tal que

$$
x_{n} \notin f^{-1}\left(\left\{x_{0}, \ldots, x_{n-1}\right\}\right) \cup\left\{f\left(x_{0}\right), \ldots, f\left(x_{n-1}\right)\right\} \cup\left\{x_{0}, \ldots, x_{n-1}\right\}
$$

O conjunto $\left\{x_{n}: n \in \omega\right\}$ é infinito, então pelo menos um dos conjuntos $\left\{x_{n}: f\left(x_{n}\right)=x_{n}\right\}$ e $\left\{x_{n}: f\left(x_{n}\right) \neq x_{n}\right\}$ é infinito. Se o primeiro é infinito satisfaz (i), se o segundo é infinito satisfaz (ii).

Teorema $2.21 \beta \mathbb{N}$ nào tem CIP, porém o produto $(\beta \mathbb{N})^{\mathfrak{c}}$ de $\mathfrak{c}$ cópias de $\beta \mathbb{N}$ onde $\mathfrak{c}$ denota o cardinal do contínuo, tem CIP.

\section{Demonstração:}

Que $(3 \mathbb{N})^{\mathfrak{c}}$ tem CIP segue do fato que $3 \mathbb{N}$ é um espaço zero-dimensional de peso $\mathfrak{c}$, do teorema (2.12) e do lema (2.15).

Para provar que $3 \mathbb{N}$ não tem $\mathrm{CIP}$, suponhamos $f: \beta \mathbb{N} \longrightarrow \beta \mathbb{N}$ uma função contínua 
$\operatorname{com} f i x(f)=\beta \mathbb{N}-\mathbb{N}$.

Se o conjunto $\{n \in \mathbb{N}: f(n) \in \beta \mathbb{N}-\mathbb{N}\}$ é infinito, seja $A=\{n \in \mathbb{N}: f(n) \in \beta \mathbb{N}-\mathbb{N}\}$. Entào $f(A) \subset(3 \mathbb{N}-\mathbb{N}) \cap f(A)$ e $f(A) \cap(\mathbb{N}-A)=\emptyset$.

Se $\{n \in \mathbb{N}: f(n) \in \beta \mathbb{N}-\mathbb{N}\}=\left\{k_{1}, \ldots, k_{m}\right\}$, seja $g: \mathbb{N} \longrightarrow \mathbb{N}$ uma função contínua tal que a restricào de $g$ a $\mathbb{N}-\left\{k_{1}, \ldots, k_{m}\right\}$ coincide com a restrição de $f$ ao mesmo conjunto. Logo, pelo lema anterior e como $f i x(f)=\beta \mathbb{N}-\mathbb{N}$, existe um conjunto $B \in \mathbb{N}$ infinito, tal que $g(x) \notin B \forall x \in B$.

Seja $A=B \cap\left(\mathbb{N}-\left\{k_{1}, \ldots, k_{m}\right\}\right)$. O conjunto $A$ assim definido é infinito e $\forall x \in A$ temos que $f(x) \notin A$, ou seja $f(x) \in \mathbb{N}-A \forall x \in A$.

Portanto $f(A) \subset \mathbb{N}-A$ e $f(A) \cap(\beta \mathbb{N}-\mathbb{N})=\emptyset$.

Ressumindo os dois casos, existe $A \subset \mathbb{N}$ infinito, tal que $f(A) \subset(\mathbb{N}-A) \cup((\beta \mathbb{N}-$ N) $\cap f(. A))$.

Os fechos considerados neste teorema são todos no espaço $\beta \mathbb{N}$.

Temos que :

$$
\begin{aligned}
\bar{A} \cap f(\bar{A}) & \stackrel{(1)}{\subset} \bar{A} \cap \overline{f(A)} \\
& \stackrel{(2)}{\subset} \bar{A} \cap(\overline{\mathbb{N}-A} \cup \overline{(\beta \mathbb{N}-\mathbb{N}) \cap f(A)} \\
& =\bar{A} \cap \overline{(\beta \mathbb{N}-\mathbb{N}) \cap f(A)} \\
& \stackrel{(3)}{\subsetneq} \bar{A} \cap(\beta \mathbb{N}-\mathbb{N}) .
\end{aligned}
$$

(1) por continuidade da função $f,(2)$ pela discussão acima, e a inclusão estrita de (3) porque a clensidade de $\bar{A} \cap(\beta \mathbb{N}-\mathbb{N})=\mathfrak{c}$ e $f(A) \cap(\beta \mathbb{N}-\mathbb{N})$ é enumerável.

Então existe $p \in \bar{A} \cap(\beta \mathbb{N}-\mathbb{N})$ tal que $p \notin \bar{A} \cap f(\bar{A})$. Como $p \in \bar{A}, p \notin f(\bar{A})$. Logo $p \notin f(\bar{A} \cap(\beta \mathbb{N}-\mathbb{N}))$, e $f(p) \neq p$, contra a suposição $f i x(f)=\beta \mathbb{N}-\mathbb{N}$.

Esta contradição mostra que $\beta \mathbb{N}$ nào tem C.IP.

Para facilitar a seguinte discussão vamos chamar $Y$ um CIP-companion de $X$ se $X \times Y$ tem CIP. e dizemos que $Y$ é um CIP-companion absoluto para uma classe $\mathcal{Q}$ de espaços se $Y$ é um CIP-companion para cada $X \in \mathcal{Q}$. 
Algums dos exemplos do capítulo são de espaços com CIP mas não são CIP- companion deles mesmos. Por exemplo a long line $L$ e a reta de Sorgenfrey $K$.

Do lema de Ward segue que $S^{1}$ é um CIP-companion absoluto para a classe dos espaços perfeitamente normais. No entanto, resulta desta seção que $S^{1}$ não é um CIP-companion absoluto para a classe dos espaços compactos. De fato vimos que $L^{*} \times S^{1}$ não tem CIP, e os espaços $\left(Z_{2}\right)^{\mathfrak{c}} \times S^{1}, \beta \mathbb{N} \times S^{1},(\beta \mathbb{N})^{\mathfrak{c}} \times S^{1}$ não tem $\mathrm{CIP}$ pelo teorema $(2.17)$.

Para certos espaços, podemos escolher um cardinal apropriado $\lambda$ de modo a obter um CIP-companion $\left(S^{1}\right)^{-1}$. Por exemplo, $\left(S^{1}\right)^{\mathfrak{c}}$ é um CIP-companion de $\left(Z_{2}\right)^{\mathfrak{c}}$ pois $\left(Z_{2}\right)^{\mathfrak{c}} \times\left(S^{1}\right)^{\mathfrak{c}}$ é homeomorfo a $\left(Z_{2} \times S^{1}\right)^{\mathfrak{c}}$ que tem CIP por teorema (2.14).

O teorema que segue mostra que todo espaço completamente regular tem CIPcompanion da forma $\left(S^{1}\right)^{-1}$.

Teorema 2.22 Seja $Y$ um espaço completamente regular. Então existe um cardinal $\lambda$ tal que $Y \times\left(S^{1}\right)^{\lambda}$ tem $C I P$.

\section{Demonstração:}

Se $Y$ for perfeitamente normal, pelo teorema (2.17) $S^{1} \times Y$ tem CIP.

Vamos supor $Y$ completamente regular e não perfeitamente normal. Podemos escolher um cardinal infinito $\lambda$ e uma base $\{B(0, \gamma): \gamma<\lambda\}$ de cozero-sets para $Y$.

Seja $\left\{Y_{\alpha}: \alpha \in \lambda\right\}$ uma família de espaços tal que

$$
Y_{0}=Y \quad \text { e } \quad Y_{\alpha}=S^{1} \quad \text { se } \alpha \neq 0
$$

Sejam também $0<\beta<\lambda, F=\left\{\alpha_{1}, \ldots, \alpha_{n}\right\}, G=\left\{\gamma_{1}, \ldots, \gamma_{n}\right\}$ onde $F, G \subset \lambda$

Definimos uma função $h$ satisfazendo as hipóteses do teorema (2.12). Temos dois casos a considerar.

caso 1: se $0 \notin F$, definimos $h$ como no lema (2.13), pois $Y_{\alpha_{1}} \times \ldots \times Y_{\alpha_{n}} \times Y_{\beta}$ é um espaço métrico. Então

$$
\left.h(x)=H_{\beta}\left(\pi_{\{\beta\}}(x), \frac{d(x)}{1+d(x)}\right)\right)
$$


onde

$$
d(x)=\left\{\begin{array}{l}
\operatorname{dist}\left(\pi_{F}(x), Y_{\alpha_{1}} \times \ldots \times Y_{\alpha_{n}} \times Y_{\beta}-B\left(\alpha_{1}, \gamma_{1}\right) \times \ldots \times B\left(\alpha_{n}, \gamma_{n}\right)\right) \\
\text { se } \pi_{F}(x) \in B\left(\alpha_{1}, \gamma_{1}\right) \times \ldots \times B\left(\alpha_{n}, \gamma_{n}\right) \\
0 \quad \text { caso contrário }
\end{array}\right.
$$

caso 2: Se $0 \in F \operatorname{sejam} F=\left\{0, \alpha_{1}, \ldots, \alpha_{n}\right\}$ e $G=\left\{\gamma_{0}, \ldots, \gamma_{n}\right\}$

Consideramos $\rho$ uma métrica em $Y_{\alpha_{1}} \times \ldots \times Y_{\alpha_{n}}$ limitada por 1, e uma função continua $f: Y_{0} \longrightarrow I$ tal que $\left.\left.B\left(0, \gamma_{0}\right)=f^{-1}(] 0,1\right]\right)$.

Para $x \in Y_{0} \times Y_{\alpha_{1}} \times \ldots \times Y_{\alpha_{n}} \times Y_{\beta}$, sejam

$$
\begin{aligned}
& e^{i \theta}=\pi_{\{B\}}(x) \\
& d(x)=\rho\left(\pi_{F-\{0\}}(x), Y_{\alpha_{\mathrm{i}}} \times \ldots \times Y_{\alpha_{n}}-B\left(\alpha_{1}, \gamma_{1}\right) \times \ldots \times B\left(\alpha_{n}, \gamma_{n}\right)\right)
\end{aligned}
$$

Definimos a funçào $h: Y_{0} \times Y_{\alpha_{1}} \times \ldots \times Y_{\alpha_{n}} \times Y_{\beta} \longrightarrow Y_{\beta}$ por:

$$
h(x)=e^{i\left(\theta+d(x) f\left(\pi_{\{0\}}(x)\right)\right)}
$$

* $h$ é contínua pois é composição de funções contínuas.

$* h(x)=\pi_{\{B\}}(x) \Leftrightarrow \pi_{F}(x) \notin B\left(0, \gamma_{0}\right) \times B\left(\alpha_{1}, \gamma_{1}\right) \times \ldots \times B\left(\alpha_{n}, \gamma_{n}\right):$

Se $\pi_{F}(x) \in B\left(0, \gamma_{0}\right) \times B\left(\alpha_{1}, \gamma_{1}\right) \times \ldots \times B\left(\alpha_{n}, \gamma_{n}\right)$, então

$$
\begin{aligned}
& \text { (1) } \pi_{F-\{0\}}(x) \in B\left(\alpha_{1}, \gamma_{1}\right) \times \ldots \times B\left(\alpha_{n}, \gamma_{n}\right) \\
& \text { (2) } \pi_{\{0\}}(x) \in B\left(0, \gamma_{0}\right)
\end{aligned}
$$

De (1) temos que $d(x)>0$ e de (2) temos que $\left.\left.\pi_{\{0\}}(x) \in f^{-1}(] 0,1\right]\right)$ e portanto que $f\left(\pi_{\{0\}}(x)\right)>0$. Logo $1 \geq d(x) f\left(\pi_{\{0\}}(x)\right)>0 \quad$ e $h(x) \neq e^{i \theta}=\pi_{\{\beta\}}(x)$.

Se $\pi_{F}(x) \notin B\left(0, \gamma_{0}\right) \times B\left(\alpha_{1}, \gamma_{1}\right) \times \ldots \times B\left(\alpha_{n}, \gamma_{n}\right)$ então

$$
\begin{aligned}
& \pi_{\{0\}}(x) \notin B\left(0, \gamma_{0}\right) \\
& \text { ou } \\
& \pi_{F-\{0\}}(x) \notin B\left(\alpha_{1}, \gamma_{1}\right) \times \ldots \times B\left(\alpha_{n}, \gamma_{n}\right)
\end{aligned}
$$

Entào $d(x) f\left(\pi_{\{0\}}(x)\right)=0 \quad$ e $\quad h(x)=e^{i \theta}=\pi_{\{\beta\}}(x)$.

Vimos que $\lambda .\left\{Y_{\alpha}: \alpha \in \lambda\right\}$ e $\{B(\alpha, \gamma): \gamma \in \lambda\}$ estão nas condições do teorema (2.12). Portanto $Y \times S^{1}=\prod\left\{Y_{\alpha}: \alpha \in \lambda\right\}$ tem CIP. 
Lema 2.23 Suponhamos que X é um espaço com um subconjunto fechado discreto de cardinalidade $\lambda$ e tal que $2^{\lambda}$ é maior que a cardinalidade da coleção de todas as funçòes contínuas de $X \mathrm{em} X$. Então $X$ não tem CIP.

\section{Demonstração:}

Seja $D$ um subconjunto discreto de $X$ de cardinalidade $\lambda$. Como subconjuntos diferentes de $D$ tem fechos diferentes em $X$, temos pelo menos $2^{\lambda}$ fechados diferentes em $X$. Então para $X$ ter C'IP devem existir no mínimo $2^{\lambda}$ funções de $X$ em $X$.

O seguinte resultado mostra que nào existe um CIP-companion absoluto completamente regular para a classe dos espaços completamente regulares.

Teorema 2.24 Para cada espaço C completamente regular existe um espaço X completamente regular tal que $\mathrm{X} \times \mathrm{C}$ nào tem $\mathrm{CIP}$.

\section{Demonstração:}

Suponhamos que $C$ é um espaço completamente regular de cardinalidade $\alpha$. Seja $\lambda=2^{\alpha}$ (a cardinalidade de partes de $C$ ).

$\left(Z_{2}\right)^{\lambda}$ possui um subconjunto denso $D$ de cardinalidade $\alpha$ (pois a densidade de $\left(Z_{2}\right)^{\wedge}$ é $\log \lambda \leq \alpha$, ver [Hod84]).

$\left(Z_{2}\right)^{\lambda}$ possui um subconjunto discreto $E$ de cardinalidade $\lambda$. (basta considerar a imersão $\lambda \longrightarrow\left(Z_{2}\right)^{\lambda}, \lambda$ como espaço discreto).

Seja $X=D \cup E . X \times C$ é um espaço completamente regular com um subconjunto denso $(D \times C)$ de cardinalidade $\alpha$ e com um subconjunto discreto $(E \times\{c\}$ onde $c \in C$ ) de cardinalidade $\lambda$.

Mais ainda, o conjunto $A=\{f: X \times C \longrightarrow X \times C: f$ é contínua $\}$ tem cardinalidade $\lambda$ pois $|D \times C|=\alpha,|X \times C|=\lambda, \operatorname{logo}|A|=\lambda^{\alpha}=\left(2^{\alpha}\right)^{\alpha}=2^{\alpha}=\lambda$.

Como $2^{\lambda}>\lambda$, pelo lema (2.23) temos que $\mathrm{X} \times C$ não tem CIP. 


\subsection{Resultados independentes}

Nesta seção vamos discutir a CIP no contexto de espaços não metrizáveis. Trabalharemos com a Reta de Sorgenfrey

Teorema 2.25 Seja $K$ a reta de Sorgenfrey. Então:

(i) $\mathrm{i}$ tem CIP

(ii) $K^{2}$ não tem CIP

(iii) Para cada inteiro $n \geq 2, K^{n}$ não tem CIP

(iv) $K^{\omega}$ não tem CIP

(v) Para cada cardinal $\lambda \geq \mathfrak{c}, \boldsymbol{K}^{\lambda}$ tem CIP

\section{Demonstração:}

(i) Todo subconjunto aberto de $K$ pode-se escrever como união enumerável disjunta de intervalos da forma $[a, b)$.

Para cada intervalo $[a, b)$, consideramos a função $f_{[a, b)}:[a, b) \longrightarrow[a, b)$ dada por $f_{[a, b)}(x)=(x+b) / 2$.

$f_{[a, b)}$ é um homeomorfismo de $[a, b)$ em $f_{[a, b)}([a, b))$, sem pontos fixos.

Se $A$ é um subconjunto fechado de $K$, então $K-A$ é aberto. Logo $K-A=\bigcup_{i=1}^{\infty}\left[a_{i}, b_{i}\right)$ onde $\left[a_{i}, b_{i}\right) \cap\left[a_{j}, b_{j}\right)=\emptyset$ se $i \neq j$. Seja $f: K \longrightarrow K$ definida por

$$
f(x)= \begin{cases}f_{\left[a_{i}, b_{i}\right)}(x) & \text { se } x \in\left[a_{i}, b_{i}\right) \\ x & \text { se } x \in A\end{cases}
$$

$f$ é contínua :

* Seja $x \in K-A$. Existe um único $i<\omega$ tal que $x \in\left[a_{i}, b_{i}\right)$ e $f_{\left[a_{i}, b_{i}\right)}$ é contínua. Portanto $f$ é contínua em $x$.

* Seja $x \in A$ e seja $\varepsilon>0$. Se $[x, x+\varepsilon) \subset A$, então $\left.f\right|_{(x, x+\varepsilon)}$ é a identidade e portanto $f$ é contínua em $x$. Se $\varepsilon>0[x, x+\varepsilon) \cap K-A \neq \emptyset$, existe $j \in \omega$ tal que $x<a_{j}<x+\varepsilon$. Entào $f\left(\left[x, a_{j}\right)\right) \subset\left[x, a_{j}\right) \subset[x, x+\varepsilon)$ : 
se $y \in\left[x, a_{j}\right) \cap A \Rightarrow f(y)=y \in\left[x, a_{j}\right)$

se $y \in\left[x, a_{j}\right) \cap K-A \Rightarrow y \in\left[a_{i}, b_{i}\right)$ para algum $i \in \omega$ tal que $x<a_{i}<b_{i}<a_{j} \Rightarrow$ $f(y) \in\left[a_{i}, b_{i}\right) \subset\left[x, a_{j}\right)$.

fix $(f)=A$ por construçào.

Então $K$ tem C'IP.

(ii) $K^{-2}$ é separável e tem cardinalidade $2^{\omega}$, então existem unicamente $\left(2^{\omega}\right)^{\omega}=2^{\omega}$ funções contínuas de $K^{-2}$ em $K^{-2}$.

O conjunto $D=\{(x,-x): x \in K\}$ é um subespaço discreto de $K^{-2}$, de cardinalidade $2^{\omega}$. Como $2^{2^{\omega}}>2^{\omega}$, pelo lema (2.23) segue que $K^{-2}$ não tem CIP.

(iii) e (iv) como em (ii), $K^{\star n}$ e $K^{\omega}$ são espaços separáveis de cardinalidade $2^{\omega}$, e possuem um subconjunto discreto de cardinalidade $2^{\omega}$. Logo pelo lema (2.23) não tem CIP.

(v) $K$ é um espaço zero-dimensional de peso $2^{\omega}$. Então pelo lema (2.15) e teorema (2.12) $\left(\lambda \geq 2^{\omega}\right) K^{-\lambda}$ tem CIP.

Ainda nào foi determinada a relaçào entre CIP e todas as potências de $K$. Em particular nào foi respondido se $K^{\prime \omega_{1}}$ tem CIP.

Teorema 2.26 Seja $K$ a reta de Sorgenfrey. Então

(i) CH implica que $\mathrm{K}^{-\infty}$ tem CIP, e

(ii) $M A+(\neg C H)$ implica que $K^{\omega_{1}}$ não tem CIP.

\section{Demonstração:}

(i) $\omega_{1}=2^{\omega}$ entào $K^{\omega_{1}}$ tem CIP pelo teorema anterior.

(ii) $M A+\left(\neg C^{\prime} H\right)$ implica que $2^{\omega_{1}}=2^{\omega}$, portanto $2^{2^{\omega}}>2^{\omega_{1}} . K^{\omega_{1}}$ possui um subconjunto discreto de cardinalidade $2^{\omega}\left(\left\{\{-x\} \times \pi_{1<\alpha<\omega_{1}}\{x\}: x \in K\right\}\right.$ é discreto $) \mathrm{e}$ é separável pelo teorema de Hewitt-Marczewski-Pondiczery. 
Então pelo lema (2.23) $K^{\omega_{1}}$ nào tem C.IP.

O seguinte é um exemplo de que a CIP não é conservada pelo produto de espaços perfeitamente normais.

Exemplo 2.27: $\left(K^{r} \times S^{1}\right)^{2}$ nâo tem CIP.

$\Lambda \times S^{1}$ é um espaço perfeitamente normal (pois $K$ é perfeitamente normal e $S^{1}$ é métrico) com a propriedade $W($ forte $)$. Então $K \times S^{1}$ tem CIP por lema de Ward. $\left(K \times S^{1}\right)^{2}$ é homeomorfo a $K \times K \times S^{1} \times S^{1}$ que é separável de cardinalidade $2^{\omega}$ e contém um subespaço discreto de cardinalidade $2^{\omega}$. Logo pelo lema $(2.23)\left(K \times S^{1}\right)^{2}$ não tem CIP. 


\section{Capítulo 3}

\section{A CIP em produtos não}

\section{enumeráveis de espaços lineares}

O objetivo deste capítulo é ver a demonstração de que $[0,1]^{\kappa}$ não tem CIP, onde $\kappa$ é um cardinal não enumerável. A prova va a ser feita para um caso mais geral: o produto não enumerável de subespaços compactos convexos de espaços lineares normados.

\subsection{Algumas definições}

Cada $X_{\alpha}$ denota um subespaço convexo e compacto com mais de um ponto, de algum espaço linear normado $\left(L_{\alpha},\|\|_{\alpha}\right)$. Observamos que cada espaço $X_{\alpha}$ é separável.

Se $A \subseteq \kappa$ e $x \in \prod_{\alpha<\kappa} X_{\alpha}$, denotamos por $x_{A}$ ou $\pi_{A}(x)$ a projeção de $\mathrm{x}$ sobre as coordenadas pertencentes ao conjunto $\mathrm{A}$. Nas mesmas condições para $\mathrm{A}, \mathrm{X}^{A}$ representa o espaço produto $\prod_{\alpha \in A} X_{\alpha}$, e portanto $x_{A} \in X^{A}$. Ás vezes escrevemos $x(\alpha)$ ao invés de $x_{\{\alpha\}}$. 
Se $A \cap B=\emptyset, x_{A} \in X^{A}$ e $x_{B} \in X^{B}$ definimos $x_{A}-x_{B} \in X^{A \cup B}$ por: $\left(x_{A}-x_{B}\right)_{A}=x_{A}$ e $\left(x_{A}-x_{B}\right)_{B}=x_{B}$.

Se $A \subseteq \kappa$ é enumerável, então $X^{A}$ é um espaço métrico. A métrica considerada no espaço $X^{A}$ é a seguinte:

$$
\rho^{A}(x, y)=\sum_{\alpha \in A} \frac{\|x(\alpha)-y(\alpha)\|_{\alpha}}{2^{f_{A}(\alpha)}}
$$

Onde $f_{A}: A \longrightarrow \omega-\{0\}$ é uma função injetora.

Para cada $\alpha$, consideramos $\rho^{\{\alpha\}}$ uma métrica limitada por 1 em $L_{\alpha}$. Logo $\rho^{A}$ é tal que: $\rho^{A}(x, y) \leq 1 \forall x, y \in X^{-A}$

Por $B^{A}(a, r)$ denotamos a bola de centro $a$ e raio $r \in \mathbb{R}$ em $X^{A}$ com a métrica $\rho^{A}$. Sabemos também que $X_{\alpha}$ é um espaço linear e convexo, portanto podemos considerar a seguinte combinação convexa de pontos de $X^{A}$ :

$$
[t x+(1-t) y](\alpha)=t x(\alpha)+(1-t) y(\alpha) \in X_{\alpha}^{r} \text { para } t \in[0,1], x, y \in X^{A} \text { e } \alpha \in A
$$

Observemos que para cada $\alpha \in A$ e para todo $t$ no intervalo $[0,1]$ temos:

$$
\|x(\alpha)-[t x(\alpha)+(1-t) y(\alpha)]\|_{\alpha}=(1-t)\|x(\alpha)-y(\alpha)\|_{\alpha} \leq\|x(\alpha)-y(\alpha)\|_{\alpha}
$$

Logo, pela definição da métrica $\rho^{A}$

$$
\rho^{A}(x, t x+(1-t) y) \leq \rho^{A}(x, y) \quad \forall t \in[0,1] e \forall x, y \in X^{A}
$$

Por último, se $F \subseteq X^{A}, C l_{A}(F)$ denotará o fecho de $\mathrm{F}$ em $X^{A}$.

Definição 3.1 Sejam $\kappa$ um cardinal infinito $e A \in[\kappa]^{\omega}$. Para cada $\alpha \in \kappa X_{\alpha}$ seja $X_{\alpha}$ um espaço topológico. Se $f: \prod_{\alpha<\kappa} X_{\alpha} \longrightarrow X$ é uma função contínua, dizemos que $f$ depende de $A$ se e somente se $\forall x, y \in \prod_{\dot{\alpha}<\kappa} X_{\alpha}\left(\pi_{A}(x)=\pi_{A}(y) \Rightarrow f(x)=f(y)\right)$.

Definição 3.2 Sejam $f: \prod_{\alpha<\kappa} X_{\alpha} \longrightarrow \prod_{\alpha<\kappa} X_{\alpha}$ uma funçào contínua, $e$ $f_{A}: \prod_{\alpha<\kappa} X_{\alpha} \longrightarrow \prod_{\alpha \in A} X_{\alpha}$ a função $f_{A}=\pi_{A} \circ f$. Se $f_{A}$ depende de $A$, definimos a função $f^{A}: \prod_{a \in A} X_{\alpha} \longrightarrow \prod_{\alpha \in A} X_{\alpha}$ como segue:

$$
f^{A}\left(\pi_{A}(x)\right)=\pi_{A}(f(x))
$$




\subsection{Lemas Geométricos}

Lema 3.3 Seja $A \in[k]^{\omega}, \alpha \in A, f: X^{A} \longrightarrow X^{A}$ contínua, $U$ um subconjunto aberto de $X_{\alpha}$. Suponhamos que existe um ponto $a \in U$ tal que $\pi_{\{\alpha\}}(f i x(f)) \cap U=\{a\} \quad \epsilon$ que $\Gamma$ é uma família de funções de $\mathrm{X}^{*} \mathrm{em} \mathrm{X}^{\mathrm{A}}$ tal que:

(i) $\forall \varepsilon>0 \quad \exists f_{\varepsilon} \in \Gamma \quad \forall x \in \pi_{\{\alpha\}}^{-1}(U) \rho^{A}\left(f(x), f_{\varepsilon}(x)\right)<\varepsilon$

(ii) $\forall \varepsilon>0 \quad \pi_{\{\alpha\}}\left(f i x\left(f_{\varepsilon}\right)\right) \cap U=\emptyset$

então existe uma função $g: X^{A} \longrightarrow X^{A}$ tal que:

(iii) $\pi_{\{\alpha\}}($ fix $(g)) \cap U=\emptyset$

(iv) $\forall x \in X^{A}(x(\alpha) \notin U \Rightarrow g(x)=f(x))$

\section{Demonstração:}

Sejam $r_{1} r_{2}$ reais tais que $r_{1}>r_{2}>0$ e $B^{\{\alpha\}}\left[a, r_{1}\right]$ e $B^{\{\alpha\}}\left[a, r_{2}\right]$ estão contidas em $U$.

Fixamos duas funçòes contínuas $\beta, \gamma: I \longrightarrow I$ tais que:

$$
\begin{array}{ll}
\beta(t)+\gamma(t)=1 & \text { para } t \in I \\
\beta(t)=0 & \text { para } t \geq r_{1} \\
\gamma(t)=0 & \text { para } t \leq r_{2}
\end{array}
$$

Consideramos também a função $\varphi:\left\{x \in X^{A}: r_{1} \geq \rho^{\{\alpha\}}(x(\alpha), a) \geq r_{2}\right\} \longrightarrow \mathbb{R}_{+}$ definida por $\rho(x)=\rho^{A}(x, f(x))$.

Vemos primeiro que $\varphi$ é positiva pois $\pi_{\{\alpha\}}(\operatorname{dom}(\varphi)) \subseteq U-\{a\}$ e por hipótese $\pi_{\{\alpha\}}(f i x(f)) \cap U=\{a\}$, portanto $\pi_{\{\alpha\}}(\operatorname{dom}(\varphi)) \cap \pi_{\{\alpha\}}($ fix $(f))=\emptyset$.

Logo para cada $x \in \operatorname{dom}(\varphi)$, temos $\rho^{\{\alpha\}}(x, f(x))>0$ e então $\varphi(x)>0$.

$\varphi$ é contínua:

$\varphi$ é composição de duas funções contínuas, a função $\chi: \operatorname{dom}(\varphi) \longrightarrow X^{A} \times X^{A}$ definida por $\chi(x)=(x, f(x))$, e a funçào $\rho^{A}$.

$\chi$ é contínua pois $\pi_{1} \circ \gamma=i d$ e $\pi_{2} \circ \gamma=f$ são funções contínuas.

$X^{A}$ é compacto e $\operatorname{dom}(\varphi)$ é um subconjunto fechado de $X^{A}$, então dom( $\left.\varphi\right)$ é compacto. 
Segue que $i m(\varphi)$ é um subconjunto compacto de $R_{+}$e portanto existe $\delta>0$ tal que $\varphi(x)>\delta \quad \forall x \in \operatorname{dom}(\varphi)$.

Seja $\varepsilon, 0<\varepsilon \leq \delta$, e definimos $g: X^{A} \longrightarrow X^{A}$ como segue:

$$
g(x)=\beta(t) f_{\varepsilon}(x)+\gamma(t) f(x) \text { onde } t=\rho^{\{\alpha\}}(x(\alpha), a)
$$

Como $\mathrm{X}^{A}$ é convexo, $f_{\varepsilon} \in X^{A}, f(x) \in X^{A}$ e $g(x)$ está no segmento de extremos $f_{\varepsilon}(x)$ e $f(x), g(x) \in X^{A} \quad \forall x \in X^{A}$.

Falta provar que $g$ satisfaz as condições (iii) e $(i v)$ do enunciado.

Para provar (iv) seja $x \in X^{A}$ tal que $x(\alpha) \notin U$, então $\rho^{\{\alpha\}}(x(\alpha), a)>r_{1}$.

Mas $\beta$ restrita ao intervalo $\left[r_{1}, 1\right]$ é a função nula então $g(x)=f(x)$.

Para provar (iii) seja $x \in X^{A}$ tal que $x(\alpha) \in U$, basta ver que $x(\alpha) \notin \pi_{\{\alpha\}}($ fix $(g))$.

Consideramos três casos.

caso 1: $\rho^{\{\alpha\}}(a, x(\alpha)) \geq r_{1}$

Como $t \geq r_{1}$ temos que

$$
\beta(t)=0 \Rightarrow g(x)=f(x)
$$

$\pi_{\{\alpha\}}($ fix $(f)) \cap U=\{a\}$, mas $x(\alpha) \neq a$ então $g(x) \neq x$.

caso 2: $\rho^{\{\alpha\}}(a, x(\alpha)) \leq r_{2}$

Como $t \leq r_{2}$ temos que

$$
\gamma(t)=0 \Rightarrow g(x)=f_{\varepsilon}(x)
$$

$\pi_{\{\alpha\}}\left(f i x\left(f_{\varepsilon}\right)\right) \cap U=\emptyset$ então $g(x) \neq x$.

caso 3: $r_{2}<\rho^{\{\alpha\}}(a, x(\alpha))<r_{1}$

Como $t=\rho^{\{\alpha\}}(a, x(\alpha)) \in I$ e $x(\alpha) \in U$, pela desigualdade (3.2) e por $(i)$ temos:

$$
\rho^{A}\left(f(x), \beta(t) f_{\varepsilon}(x)+\gamma(t) f(x)\right) \leq \rho^{A}\left(f(x), f_{\varepsilon}(x)\right)<\varepsilon \Rightarrow \rho^{A}(f(x), g(x))<\varepsilon
$$

Mas $x \in \operatorname{dom}(\varphi)$, e portanto $\rho^{A}(f(x), x)>\delta \geq \varepsilon$. Segue que:

$$
\begin{aligned}
\rho^{A}(g(x), x) & \geq \rho^{A}(f(x), x)-\rho^{A}(f(x), g(x))>\delta-\varepsilon \geq 0 \\
& \Rightarrow \rho^{A}(g(x), x)>0 \Rightarrow g(x) \neq x
\end{aligned}
$$


Lema 3.4 Sejam $A \in[i]^{\omega}, \alpha \in A, f: X^{A} \longrightarrow X^{A}$ uma funçào contínua. Suponhamos dados $p, q \in X_{\alpha}, p \neq q$ e duas famílias $(\Phi, \Gamma)$ de funçóes de $X^{A} \mathrm{em} X^{A}$ tais que:

(i) $\forall \varepsilon>0 \exists f_{\varepsilon} \in \Phi, \exists g_{\varepsilon} \in \Gamma$ tais que $\forall x \in X^{A} \quad \rho^{A}\left(f(x), f_{\varepsilon}(x)\right)<\varepsilon e$ $\rho^{A}\left(f(x), g_{\varepsilon}(x)\right)<\varepsilon$

(ii) $\forall \varepsilon>0 \pi_{\{\alpha\}}\left(f i x\left(f_{\varepsilon}\right)\right)=\{p\} \quad$ e $\quad \pi_{\{\alpha\}}\left(\right.$ fix $\left.\left(g_{\varepsilon}\right)\right)=\{q\}$

Entào existe $r \in X_{\alpha}$ tal que $r \in \pi_{\{\alpha\}}(f i x(f)) \operatorname{mas} r \notin\{p, q\}$.

\section{Demonstração:}

Suponhamos que $\pi_{\{\alpha\}}(f i x(f)) \subseteq\{p, q\}$. Vamos chegar a uma contradição usando duas vezes o lema (3.3).

Escolhemos dois abertos $U_{p}, U_{q}$ com fechos disjuntos, tais que $p \in U_{p} \subseteq X_{\alpha}$, $q \in U_{q} \subseteq X_{\alpha}$. Se $q \in \pi_{\alpha}(f i x(f)), f, \Phi$ e $U_{q}$ estão nas condições de hipóteses do lema (3.3). Obtemos então uma função $g: X^{A} \longrightarrow X^{A}$ tal que:

$$
\left\{\begin{array}{l}
\pi_{\{\alpha\}}(f i x(g)) \cap U_{q}=\emptyset \\
\forall x \in X^{A} \operatorname{com} x(\alpha) \notin U_{q}, g(x)=f(x)
\end{array}\right.
$$

Se $q \notin \pi_{\{\alpha\}}(f i x(f))$, seja $g=f$.

Em ambos casos $\pi_{\{\alpha\}}($ fix $(g)) \subseteq\{p\}$ e $\forall x \in X^{A}$ tal que $x(\alpha) \in \overline{U_{p}}, g(x)=f(x)$.

Aplicando de novo o lema 3.3, desta vez para $g$, $\Gamma$ e $U_{p}$, obtemos una função $h: X^{A} \longrightarrow X^{A}$ tal que:

$$
\left\{\begin{array}{l}
\pi_{\{\alpha\}}(\text { fix }(h)) \cap U_{p}=\emptyset \\
\forall x \in X^{A} \operatorname{com} x(\alpha) \notin U_{p}, h(x)=g(x)
\end{array}\right.
$$

Se $r \in \pi_{\{\alpha\}}($ fix $(h))$, entào $r \notin U_{p}$, e como $\pi_{\{\alpha\}}(f i x(g)) \subset\{p\}$, temos que $f i x(h)=\emptyset$. Por outro lado sabemos que $h$ é uma função contínua e que para todo $\alpha \in A \quad X_{\alpha}$ é um espaço linear normado. compacto e convexo, entào $h$ tem ponto fixo, i.e. $f i x(h) \neq \emptyset$ (ver apêndice: teorema de Shauder). 
Definição 3.5 Sejam $B \subseteq A, x_{B} \in X^{B}, f: X^{A} \longrightarrow X^{A}$. Definimos a funçào $f_{x_{B}}: X^{A-B} \longrightarrow X^{A-B}$ por :

$$
f_{x_{B}}\left(y_{A-B}\right)=\left(f\left(x_{B}-y_{A-B}\right)\right)_{A-B}
$$

Lema 3.6 Sejam $A, B \in[\kappa]^{\omega}, B \subseteq A \quad f: X^{A} \longrightarrow X^{A}$ tais que existe $x \in$ fix $(f) e$ a funçào $f_{B}=\pi_{B} f$ depende de $B$. Se $y_{A-B}$ é um ponto fixo de $f_{x_{B}}$, então $x_{B} \frown y_{A-B}$ é um ponto fixo de $f$, onde $x_{B}=\pi_{B}(x)$.

\section{Demonstração:}

Seja $x$ um ponto fixo de $f$, então $f_{B}(x)=(f(x))_{B}=x_{B}$ $f_{B}$ depende de $B, \log 0 \quad\left(f\left(x_{B}-y_{A-B}\right)\right)_{B}=(f(x))_{B}=x_{B}$. Por outro lado temos que $y_{A-B}=f_{x_{B}}\left(y_{A-B}\right)=\left(f\left(x_{B} \frown y_{A-B}\right)\right)_{A-B}$.

Portanto $f\left(x_{B} \frown y_{A-B}\right)=x_{B} \frown y_{A-B}$.

Lema 3.7 Sejam $A, B \in[\kappa]^{\omega}, B \subseteq A, \alpha \in A-B, F \subseteq X^{A}$ fechado, $p_{0}, p_{1} \in X_{\alpha}$, $p_{0} \neq p_{1}$. Sejam também $F_{p_{0}}$ e $F_{p_{1}}$ subconjuntos de $F$ tais que $\overline{\pi_{B}\left(F_{p_{0}}\right)} \cap \overline{\pi_{B}\left(F_{p_{1}}\right)} \neq \emptyset$ e com as seguintes propricdades:

(i) $\pi_{\{a\}}\left(\pi_{B}^{-1}\left(\pi_{B}\left(F_{p_{i}}\right)\right) \cap F\right)=\left\{p_{i}\right\}$ para $i=0,1$

(ii) $\pi_{\{\alpha\}}(F) \subseteq\left\{p_{0}, p_{1}\right\}$

Suponhamos que $f: X^{A} \longrightarrow X^{A}$ é uma função contínua tal que $f_{B}$ depende de $B e$ para todo $x \in F$ tem-se $f(x)=x$.

Então existe $x \in X^{A}-F$ tal que $f(x)=x$.

\section{Demonstração:}

Vamos supor, por contradição, que $f(x)=x \Leftrightarrow x \in F$.

Observações:

Seja $x \in F$. Consideramos o conjunto de todos os pontos fixos de $f_{x_{B}}$. Pelo lema (3.6), se $y_{A-B} \in f i x\left(f_{x_{B}}\right)$, então $x_{B}-y_{A-B} \in f i x(f)$. Nesse caso, pela suposição de que $x \in f i x(f) \Leftrightarrow x \in F$, temos que $x_{B} \frown y_{A-B} \in F$. 
Por hipótese $F_{p_{\boldsymbol{t}}} \subseteq F$ para $i=0,1$, então se $x \in F_{p_{i}}$ e $y_{A-B} \in f i x\left(f_{x_{B}}\right)$ temos que $x_{B}-y_{A-B} \in F$. Porém também temos que $x_{B}-y_{A-B} \in \pi_{B}^{-1}\left(\pi_{B} F_{p_{i}}\right)$. Segue que $x_{B}-y_{A-B} \in\left(\pi_{B}^{-1}\left(\pi_{B} F_{p_{i}}\right)\right) \cap F$ e portanto que $\pi_{\{\alpha\}}\left(x_{B}-y_{A-B}\right) \in \pi_{\{\alpha\}}\left(\pi_{B}^{-1}\left(\pi_{B} F_{p_{i}}\right) \cap F\right)$. Entào por $(i)$ temos $\pi_{\{\alpha\}}\left(\right.$ fix $\left.\left(f_{x_{B}}\right)\right) \subseteq\left\{p_{i}\right\}$.

Em geral por (ii) temos $\pi_{\{\alpha\}}\left(f i x\left(f_{x_{B}}\right)\right) \subseteq\left\{p_{0}, p_{1}\right\} \forall x \in F$. pois se $y_{A-B} \in f i x\left(f_{x_{B}}\right)$, então $x_{B} \frown y_{A-B} \in F$. e como $\alpha \in A-B \pi_{\{\alpha\}}\left(x_{B} \frown y_{A-B}\right)=\pi_{\{\alpha\}}\left(y_{A-B}\right)$.

Seja $z \in F$ tal que $z_{B} \in \overline{\pi_{B}\left(F_{p_{0}}\right)} \cap \overline{\pi_{B}\left(F_{p_{1}}\right)}$. Existe $z$ com estas características pois:

(1) $\overline{\pi_{B}\left(F_{p_{0}}\right)} \cap \overline{\pi_{B}\left(F_{p_{1}}\right)} \neq \emptyset$

(2) F é compacto e $\pi_{B}\left(F_{p_{\imath}}\right) \subseteq \pi_{B}(F) \operatorname{logo} \overline{\pi_{B}\left(F_{p_{\imath}}\right)} \subseteq \overline{\pi_{B}(F)}=\pi_{B}(F)$.

Vamos aplicar o lema (3.4) para: $f_{z_{B}}: X^{A-B} \longrightarrow X^{A-B}, \Phi=\left\{f_{x_{B}}: x \in F_{p_{0}}\right\}$ e $\Gamma=\left\{f_{x_{B}}: x \in F_{p_{1}}\right\}$.

Existe entào, $r \in X^{\prime \alpha}, r \notin\left\{p_{0}, p_{1}\right\}$ tal que $r \in \pi_{\{\alpha\}}\left(f i x\left(f_{z_{B}}\right)\right)$ i.e $\exists y_{A-B} \in f i x\left(f_{z_{B}}\right)$ com $\pi_{\{\alpha\}}\left(y_{A-B}\right)=r$. Portanto $\pi_{\{\alpha\}}\left(z_{B} \frown y_{A-B}\right) \not \subset\left\{p_{0}, p_{1}\right\}, \log 0 z_{B} \frown y_{A-B} \notin F$. Mas como $y_{A-B} \in \operatorname{fix}\left(f_{z_{B}}\right)$ e $z \in F$, temos que $z_{B}-y_{A-B} \in F$.

Chegamos entào a contradição buscada.

Só resta provar que $f_{\Sigma_{B}}$. $\Phi, \Gamma$ estão nas condições do lema (3.4).

Chamando de $f_{\varepsilon}$ aos elementos de $\Phi$ e de $g_{\varepsilon}$ aos elementos de $\Gamma$ temos que $\forall \varepsilon>0$

$$
\begin{aligned}
& \pi_{\{\alpha\}}\left(f i x\left(f_{\varepsilon}\right)\right)=\left\{p_{0}\right\} \\
& \mathrm{e} \\
& \pi_{\{\alpha\}}\left(f i x\left(g_{\varepsilon}\right)\right)=\left\{p_{1}\right\}
\end{aligned}
$$

Portanto a condição (ii) do lema (3.4) foi verificada nas observaçòes do início da prova.

Para provar a condição (i) fixamos $\varepsilon>0$. Como f é contínua e $X^{A}$ é compacto, $f$ é uniformemente contínua em $X^{A}$. Entào existe $\delta>0$ tal que $\rho^{A}\left(f(x), f\left(x^{\prime}\right)\right)<\varepsilon$ sempre que $\rho^{-1}\left(x, x^{\prime}\right)<\delta$.

$z_{B} \in \overline{\pi_{B}\left(F_{p_{0}}\right)}$ portanto existe $x \in F_{p_{0}}$ tal que $\rho^{B}\left(z_{B}, x_{B}\right)<\delta$. 
Afirmação: $\rho^{A-B}\left(f_{z_{B}}\left(y_{A-B}\right), f_{x_{B}}\left(y_{A-B}\right)\right)<\varepsilon \forall y_{A-B} \in X^{A-B}$

prova:

$\forall y_{A-B} \in X^{A-B} \rho^{A}\left(z_{B}-y_{A-B}, x_{B}-y_{A-B}\right)=\rho^{B}\left(z_{B}, x_{B}\right)<\delta$. Segue que $\rho^{A}\left(f\left(z_{B}-y_{A-B}\right), f\left(x_{B}-y_{A-B}\right)\right)<\varepsilon$, então $\rho^{A-B}\left(f_{z_{B}}\left(y_{A-B}\right), f_{x_{B}}\left(y_{A-B}\right)\right)<\varepsilon$.

Logo a condição (i) é satisfeita por $\Phi$. Analogamente $(i)$ é satisfeita por $\Gamma$.

Lema 3.8 Seja $A \in[\kappa]^{\omega}$. Suponha que $F \subseteq X^{\kappa}$ é fechado e que $f: X^{\kappa} \longrightarrow X^{\kappa} \quad$ é uma funçào contínua tal que $f_{A}=\pi_{A} f$ depende de $A$. Se existe $x_{A} \in\left(X^{A}-\pi_{A}(F)\right)$ tal que $f^{A}\left(x_{A}\right)=x_{A}$. Entio triste $x \in\left(X^{K}-F\right)$ tal que $x=f(x)$.

(lembramos que $f^{A}\left(\pi_{A}(x)\right)=\pi_{A}(f(x))$ )

\section{Demonstração:}

Seja $x_{A} \in\left(X^{A}-\pi_{A}(F)\right)$ tal que $f^{A}\left(x_{A}\right)=x_{A}$ e seja $f_{x_{A}}: X^{\kappa-A} \longrightarrow X^{\kappa-A}$.

$f_{x_{A}}$ é contínua e para cada $\alpha \in \kappa-A, X_{\alpha}$ é um espaço compacto, convexo, linear normado, então $f_{x_{A}}$ tem ponto fixo (ver apêndice: teorema A.S).

Seja $y_{\kappa-A}$ ponto fixo de $f_{x_{A}}$. Temos que:

(1) $f_{x_{A}}\left(y_{\kappa-A}\right)=\left(f\left(x_{A}-y_{\kappa-A}\right)\right)_{\kappa-A}=y_{\kappa-A}$

(2) $f^{A}\left(x_{A}\right)=x_{A}$ por hipótese, mas $f^{A}\left(x_{A}\right)=f\left(x_{A}-y_{\kappa-A}\right)_{A}$ por definição.

Concluimos de (1) e (2) que $f\left(x_{A} \frown y_{\kappa-A}\right)=x_{A} \frown y_{\kappa-A}$ i.e. $x_{A} \frown y_{\kappa-A}$ é ponto fixo de $f$.

Como $x_{A} \notin \pi_{A}(F), x_{A}-y_{\kappa-A} \notin F$.

Definição 3.9 t'm conjunto $F \subseteq 2^{\kappa}$ é chamado conjunto splitting se $F$ é fechado e existe um conjunto estacionário $S \subseteq[\kappa]^{\omega}$ tal que para cada $B \in S$ existem $a \in \kappa-B, F_{0}, F_{1} \subseteq F$ tais que:

(i) $C l_{B}\left(\pi_{B}\left(F_{0}\right)\right) \cap C l_{B}\left(\pi_{B}\left(F_{1}\right)\right) \neq \emptyset$

(ii) $\pi_{\{\alpha\}}\left(\pi_{B}^{-1}\left(\pi_{B}\left(F_{i}\right)\right) \cap F\right)=\{i\} \quad$ para $i=0,1$

Teorema 3.10 Suponhamos que $\kappa$ é um cardinal não enumerável e que para cada $\alpha<\kappa, X_{\alpha}$ é um subconjunto compacto e convexo com pelo menos dois pontos de al- 
gum espaço linear normado. Se existe um conjunto splitting $F \subseteq 2^{\kappa}$. então $\prod_{\alpha<\kappa} \mathrm{x}_{\alpha}$ nào tem CIP.

\section{Demonstração:}

Para cada $\alpha<\kappa$ escolhemos $p_{0}^{\alpha}, p_{1}^{\alpha} \in X_{\alpha}^{r}$ diferentes. Vamos trabalhar com uma cópia homeomorfa de um conjunto splitting $\mathrm{F}$ em $\prod_{\alpha<\kappa}\left\{p_{0}^{\alpha}, p_{1}^{\alpha}\right\}$ que é um subconjunto fechado de $\prod_{\alpha<\kappa} X_{\alpha}$. Mostraremos que para esta cópia denotada também por $\mathrm{F}$, não existe uma função contínua $f: \prod_{\alpha<\kappa} X_{\alpha} \longrightarrow \prod_{\alpha<\kappa} X_{\alpha}$ tal que $f(x)=x \Leftrightarrow x \in F$.

Suponhamos que $f(x)=x$ para todo $x \in F$. Seja $C_{f}=\left\{A \in[\kappa]^{\omega}: f_{A}\right.$ depende de $\left.A\right\}$. $C_{f}$ é c.u.b (ver apêndice: lema A.5). Sejam $B \in C_{f} \cap S$ e $\alpha \in \kappa-B$ onde $S$ e $\alpha$ são tomados da definiçào de conjunto splitting.

Fixamos também $A \in C_{f}$ tal que $B \subseteq A$ e $\alpha \in A$. Observamos que existe $A$ com essas caraterísticas pois $C_{f}$ é não limitado em $[\kappa]^{\omega}$.

Aplicamos o lema (3.T) para $F_{p_{i}}=\pi_{A}\left(F_{i}\right)$ e $f^{A}: X^{A} \longrightarrow X^{A}$. A função $f^{A}$ e os conjuntos $F_{p_{i}}$ estão nas condições de hipótese do lema pela definição de conjunto splitting.

Então, por lema (3.7) existe $x_{A} \notin \pi_{A}(F)$ tal que $f^{A}\left(x_{A}\right)=x_{A}$. Logo pelo lema (3.8), existe $x \notin F$ tal que $x=f(x)$.

\subsection{Construção de um conjunto splitting}

Teorema 3.11 Existe um conjunto splitting $F \subseteq 2^{\omega_{1}}$.

\section{Demonstração:}

Vamos construir conjuntos fechados $\pi_{\alpha}(F)$ por induçào sobre $\alpha \operatorname{com} \omega<\alpha \leq \omega_{1}$. Vamos requerer que:

(i) $\forall \beta<\alpha \pi_{\beta}(F)=\pi_{\beta}^{\alpha}\left(\pi_{\alpha}(F)\right)$

(ii) Se $\alpha$ é ordinal limite então $\pi_{\alpha}(F)=\left\{x \in 2^{\alpha}: \forall \beta<\alpha \quad x_{\beta} \in \pi_{\beta}(F)\right\}$ 
É uma construção por limite inverso.

Se exigirá também que $\forall \alpha, \omega \leq \alpha \leq \omega_{1}, 1_{\alpha}$ seja não isolado em $\pi_{\alpha}(F)$. Onde $1 \in 2^{\omega_{1}}$ é tal que $1(\beta)=1 \forall \beta<\omega_{1}$.

* para $\alpha=\omega$ pomos $\pi_{\omega}(F)=2^{\omega}$

* para a ordinal limite:

Suponhamos que $\forall \beta<\alpha, \pi_{\beta}(F)$ é um subconjunto fechado de $2^{\beta}$, com $1_{\beta}$ ponto não isolado de $\pi_{\beta}(F)$.

Pela condição (ii), $1_{\alpha}$ é ponto nào isolado de $\pi_{\alpha}(F)$. Caso contrário, se $1_{\alpha}$ é isolado em $\pi_{a}(F)$, existe um aberto básico $U_{\alpha}$ de $2^{\alpha}$ tal que $1_{\alpha} \in U_{\alpha}$ e $\left(U_{\alpha}-\left\{1_{\alpha}\right\}\right) \cap \pi_{\alpha}(F)=\emptyset$. Como $\alpha$ é ordinal limite, existe $\beta<\alpha$ tal que

$$
U_{\alpha}=\pi_{\beta}^{\alpha}\left(U_{\alpha}\right) \times \prod_{\beta \leq \lambda<\alpha} 2
$$

$\pi_{3}^{\alpha}\left(U_{\alpha}\right)$ é aberto de $2^{\beta}$ e $1_{\beta} \in \pi_{\beta}^{\alpha}\left(U_{\alpha}\right)$. Vamos provar que $\left(\pi_{\beta}^{\alpha}\left(U_{\alpha}\right)-\left\{1_{\beta}\right\}\right) \cap \pi_{\beta}(F)=\emptyset$ o que seria uma contradição $\operatorname{com} 1_{\beta}$ não isolado em $\pi_{\beta}(F)$.

Suponhamos que existe $y_{\beta} \in\left(\pi_{\beta}^{\alpha}\left(U_{\alpha}\right)-\left\{1_{\beta}\right\}\right) \cap \pi_{\beta}(F)$. Teríamos então que :

(1) $y_{\beta} \in \pi_{\beta}(F)=\pi_{\beta}^{\alpha}\left(\pi_{\alpha}(F)\right) \Rightarrow \exists x \in \pi_{\alpha}(F)$ tal que $x_{\beta}=y_{\beta}$

(2) $y_{3} \in\left(\pi_{\beta}^{\alpha}\left(U_{\alpha}\right)-\left\{1_{\beta}\right\}\right)$ i.e. $y_{\beta} \in \pi_{\beta}^{\alpha}\left(U_{\alpha}\right)$ e $y_{\beta} \neq 1_{\beta}$

Portanto $x \neq 1_{\alpha}$ e $x \in\left(\pi_{\beta}^{\alpha}\right)^{-1}\left(\pi_{\beta}^{\alpha}\left(U_{\alpha}\right)\right)=U_{\alpha}$. De (1) e (2), $x \in\left(U_{\alpha}-\left\{1_{\alpha}\right\}\right) \cap \pi_{\alpha}(F)=\emptyset$.

Também pela condição (ii) e por ser $F$ compacto, temos que para cada $\beta<\alpha, \pi_{\beta}(F)=$ $\pi_{3}^{\alpha}\left(\pi_{\alpha}(F)\right)$.

Falta provar que $\pi_{\alpha}(F)$ é um subconjunto fechado de $2^{\alpha}$.

Seja $x \in 2^{\alpha}-\pi_{\alpha}(F)$. Sabemos que para cada $\beta<\alpha, \pi_{\beta}(F)$ é um subconjunto fechado de $2^{\beta}$. Como $x \notin \pi_{\alpha}(F)$, existe $\beta<\alpha$ tal que $x_{\beta} \notin \pi_{\beta}(F)$ e portanto existe $U_{3}$ uma vizinhança aberta de $x_{\beta}$ em $2^{\beta}$ tal que $U_{\beta} \cap \pi_{\beta}(F)=\emptyset$.

Seja $U=U_{\beta} \times \prod_{\beta \leq \lambda<\alpha} 2$. U é um subconjunto aberto de $2^{\alpha}$ e $x \in U$.

Suponhamos que $U \cap \pi_{\alpha}(F) \neq \emptyset$ e seja $y \in U \cap \pi_{\alpha}(F)$. Como $y \in \pi_{\alpha}(F) \quad y_{\beta} \in \pi_{\beta}(F)$ e como $y \in U, \quad y_{\beta} \in U_{\beta}$. Temos então que $y_{\beta} \in U_{\beta} \cap \pi_{\beta}(F)$ o que contradiz 
$U_{3} \cap \pi_{\beta}(F)=\emptyset$.

Logo $U \cap \pi_{\alpha}(F)=\emptyset$ e $\pi_{\alpha}(F)$ é um subconjunto fechado de $2^{\alpha}$.

* Para a ordinal sucessor:

Suponhamos que $\alpha=\beta+1$ e que $\forall \gamma \leq \beta, \pi_{\gamma}(F)$ satisfaz a condição (i), com $1_{\gamma}$ não isolado em $\pi_{\gamma}(F)$.

Como $\beta$ é enumerável, $2^{\beta}$ tem uma base enumerável para $1_{\beta}$. Além disso $2^{\beta}$ é zero-dimensional, portanto existe uma sequência $\left(U_{n}^{\beta}\right)_{n \leq \omega}$ de subconjuntos abertose-fechados de $2^{3}$ tal que:

(iii) $\left(U_{n}^{\beta}\right)_{n \leq \omega}$ é uma base de vizinhanças de $1_{\beta}$ tal que

$$
U_{n+1}^{\beta} \cap \pi_{\beta}(F) \subseteq \overline{U_{n+1}^{\beta} \cap \pi_{\beta}(F)} \subseteq U_{n}^{\beta} \cap \pi_{\beta}(F) \quad \forall n \in \omega
$$

Para cada $n \in \omega$, seja $V_{n}^{\beta}=U_{n}^{\beta} \cap \pi_{\beta}(F)$

(iv) $\forall n\left(V_{n}^{\beta}-V_{n+1}^{\beta}\right) \neq \emptyset$

(v) $V_{0}^{\beta}=\pi_{\beta}(F)$.

Vamos definir $\pi_{\alpha}(F)$ estendendo $\pi_{\beta}(F)$ da seguinte forma:

Se $x_{3} \in\left(V_{2 k}^{\beta}-V_{2 k+1}^{\beta}\right)$ para algum $k \in \omega$, então $x(\beta)=0$

Se $x_{3} \in\left(V_{2 k+1}^{* 3}-V_{2 k+2}^{\beta}\right)$ para algum $k \in \omega$, então $x(\beta)=1$

Se $x_{\beta}=1_{\beta}$, então $x(\beta)$ pode ser 0 ou 1

$\pi_{\alpha}(F)$ assim definido satisfaz a condição (i):

se $x \in \pi_{\alpha}(F)$, então $x_{\beta} \in \pi_{\beta}(F)$ e portanto $\pi_{\beta}^{\alpha}\left(\pi_{\alpha}(F)\right) \subseteq \pi_{\beta}(F), \forall \beta<\alpha$.

Por $(v), x_{\beta} \in \pi_{\beta}(F) \Leftrightarrow x_{\beta} \in V_{0}^{\beta}$. Se $x_{\beta} \in V_{0}^{\beta}$, então $x_{\beta} \in\left(V_{2 k+i}^{\beta}-V_{2 k+i+1}^{\beta}\right)$ para algum $k \in \omega$ ou $x_{\beta}=1_{\beta}$. Em ambos casos $x_{\beta} \in \pi_{\beta}^{\alpha}\left(\pi_{\alpha}(F)\right)$. Logo $\pi_{\beta}(F) \subseteq \pi_{\beta}^{\alpha}\left(\pi_{\alpha}(F)\right)$

$1_{\alpha}$ é ponto não isolado em $\pi_{\alpha}(F)$ :

Suponhamos que $1_{\alpha}$ é isolado em $\pi_{\alpha}(F)$. Neste caso existe um aberto $U \subseteq 2^{\beta}$ tal que

$$
1_{\beta} \in U \text { e }\left(\pi_{\alpha}(F)-\left\{1_{\alpha}\right\}\right) \cap(U \times\{1\})=\emptyset .
$$

Por (iii), existe $n \in \omega$ tal que $U_{n}^{\beta} \times\{1\} \subseteq U \times\{1\}$. Logo $V_{n}^{\beta} \times\{1\} \subseteq\left(U \cap \pi_{\beta}(F)\right) \times\{1\}$. 
Seja $y \in\left(V_{2 n+1}^{3}-V_{2 n+2}^{\beta}\right)$. Pela definição de $\pi_{\alpha}(F), y-1 \in\left(\pi_{\alpha}(F)-\left\{1_{\alpha}\right\}\right) \cap U \times\{1\}$, contra a igualdade (1.3).

$\pi_{\alpha}(F)$ é fechado em $2^{\alpha}$ : Seja $x \in 2^{\alpha}-\pi_{\alpha}(F)$. Pode acontecer:

1. $x_{\beta} \notin \pi_{\beta}(F)$, entào existe $U$ vizinhança aberta de $x_{\beta}$ em $2^{\beta}$ tal que $U \cap \pi_{\beta}(F)=\emptyset$. Entào $U \times\{x(\beta)\}$ é vizinhança aberta de $x$ e é disjunta de $\pi_{\alpha}(F)$; ou

2. $x_{\beta} \in \pi_{\beta}(F)$ e então $x_{\beta} \in\left(U_{2 k+i}^{\beta}-U_{2 k+i+1}^{3}\right)$ para algum $k$ e $x(\beta)=1-i$ com $i \in\{0,1\}$.

Como $L_{n}^{+3}$ é aberto e fechado para cada $\mathrm{n},\left(U_{2 k+i}^{\beta}-U_{2 k+i+1}^{\beta}\right) \times\{1-i\}$ é vizinhança aberta e fechada de $x$ e $\pi_{\alpha}(F) \cap\left(U_{2 k+i}^{\beta}-U_{2 k+i+1}^{\beta}\right) \times\{1-i\}=\emptyset$.

Seja $F=\pi_{\omega_{1}}(F)$. Vamos provar que $F$ é um conjunto splitting de $2^{\omega_{1}}$.

Seja $S=\left\{B \in\left[\omega_{1}\right]^{\omega}: B \in \omega_{1}\right\}$ ou seja que $S$ é um conjunto de ordinais. $S$ é estacionário pois Ś é c.u.b de $\left[\omega_{1}\right]^{\omega}$.

Fixamos $B \in S \quad$ e consideramos $\alpha=B \quad\left(\alpha \in \omega_{1}-B\right)$. Seja

$$
F_{i}=\left\{x \in F: \pi_{\alpha}(x) \in \bigcup_{k \in \omega}\left(U_{2 k+i}^{\alpha}-U_{2 k+i+1}^{\alpha}\right)\right\}
$$

$1_{\alpha} \in C l_{B}\left(\pi_{B}\left(F_{0}\right)\right) \cap C l_{B}\left(\pi_{B}\left(F_{1}\right)\right):$

Seja $U$ uma vizinhança aberta de $1_{\alpha}$ em $2^{\alpha}$. Como $\left(U_{n}^{\alpha}\right)_{n \leq \omega}$ é uma base para $1_{\alpha}$ com $U_{n+1}^{\alpha} \subset L_{n}^{\alpha}$, existe $n_{0} \in \omega$ tal que $1_{\alpha} \in U_{n}^{\alpha} \subset U \forall n \geq n_{0}$.

$V_{n}^{\alpha}=U_{n}^{\alpha} \cap \pi_{\alpha}(F)$, entào $V_{n}^{\alpha} \subset U, \forall n \geq n_{0}$.

Seja $k \in \omega$ tal que $2 k \geq n_{0}$. Temos que $V_{2 k+i+1}^{\alpha} \subset V_{2 k+i}^{\alpha} \subset U, \operatorname{logo}\left(V_{2 k+i}^{\alpha}-V_{2 k+i+1}^{\alpha}\right) \subset U$. Por (iv), $V_{2 k+i}^{\alpha}-V_{2 k+i+1}^{\alpha} \neq \emptyset$. Portanto existe $x \in F$ tal que $\pi_{\alpha}(x) \in\left(U_{2 k+i}^{\alpha}-\right.$ $\left.U_{2 k+i+1}^{\alpha}\right) \subset U$, entào $F_{i} \cap U \neq \emptyset$ para $i=0,1$.

Para provar que $\pi_{\{\alpha\}}\left(\pi_{B}^{-1}\left(\pi_{B}\left(F_{i}\right)\right) \cap F\right)=\{i\}$ para $i=0,1$, seja $x \in \pi_{\alpha}^{-1}\left(\pi_{\alpha}\left(F_{i}\right)\right) \cap F$.

$$
\begin{aligned}
& \pi_{\alpha}(x) \in \pi_{i x}\left(F_{i}\right) \subset \bigcup_{k \in \omega}\left(\left(_{2 k+i}^{\alpha}-V_{2 k+i+1}^{\alpha}\right)\right. \\
& \text { e como } \\
& x \in F \text { temos que } \pi_{\alpha}(x) \in \bigcup_{k \in \omega}\left(V_{2 k+i}^{\alpha}-V_{2 k+i+1}^{\alpha}\right) \text { e } \pi_{\alpha+1}(x) \in \pi_{\alpha+1}(F) .
\end{aligned}
$$


portanto $\pi_{\{\alpha\}}(x)=x(\alpha)=i$. Como $\alpha=B, x(\alpha)=i \forall x \in \pi_{B}^{-1}\left(\pi_{B}\left(F_{i}\right)\right) \cap F$. Segue que $\pi_{\{\alpha\}}\left(\pi_{B}^{-1}\left(\pi_{B}\left(F_{i}\right)\right) \cap F\right)=\{i\}$ para $i=0,1$.

Lema 3.12 Se existe um conjunto splitting $F \subseteq 2^{\omega_{1}}$, então existe um conjunto splitting $H \subseteq 2^{\kappa}$ para todo cardinal não enumerável $\kappa$.

\section{Demonstração:}

Sejam $F \subseteq 2^{\omega_{1}}$ conjunto splitting e $S_{F}$ o conjunto estacionário para F. Se $B^{\prime} \in S_{F}^{\prime}$, sejam $\alpha_{B^{\prime}} \in\left(\omega_{1}-B^{\prime}\right)$ e $F_{0}^{B^{\prime}}, F_{1}^{B^{\prime}}$ nas condições da definição de conjunto splitting. afirmação: $H=F \times 2^{\kappa-\omega_{1}}$ é um conjunto splitting de $2^{\kappa}$ prova:

1) H é fechado de $2^{\kappa}$ pois $F$ é fechado de $2^{\omega_{1}}$

2) $S=\left\{B \in[\kappa]^{\omega}: B \cap \omega_{1} \in S_{F}\right\}$ é um conjunto estacionário de $[\kappa]^{\omega}$ :

Seja $C \subset[\kappa]^{\omega}$ c.u.b. Basta provar que existe $\gamma_{0} \in C$ tal que $\left(\gamma_{0} \cap \omega_{1}\right) \in S_{F}$, pois nesse caso $\gamma_{0} \in S \cap C$.

Seja $C^{\prime}=\left\{\gamma^{\prime} \in\left[\omega_{1}\right]^{\omega}: \gamma^{\prime}=\gamma \cap \omega_{1} \operatorname{com} \gamma \in C\right\}$

- C é fechado, portanto $C^{\prime}$ é fechado.

- $C^{\prime}$ é não limitado em $\left[\omega_{1}\right]^{\omega}$ :

se $\delta \in\left[\omega_{1}\right]^{\omega}$, então existe $\gamma \in C$ tal que $\delta \subseteq \gamma$, pois C é c.u.b.

Logo $\delta=\delta \cap \omega_{1} \subseteq \gamma \cap \omega_{1} \in C^{\prime}$. Segue que $C^{\prime} \subset\left[\omega_{1}\right]^{\omega}$ é c.u.b. Como $S_{F} \subset\left[\omega_{1}\right]^{\omega}$ é conjunto estacionário, temos que existe $\gamma_{0}^{\prime} \in C^{\prime} \cap S_{F}$ e então existe $\gamma_{0} \in C$ tal que $\gamma_{0}^{\prime}=\gamma_{0} \cap \omega_{1} \in S_{F}$.

3) Sejam $B \in S^{\prime}$ e $B^{\prime}=B \cap \omega_{1} \in S_{F}$, e sejam :

$$
\begin{aligned}
& F_{0}=F_{0}^{B^{\prime}} \times 2^{\kappa-\omega_{1}} \\
& F_{1}=F_{0}^{B^{\prime}} \times 2^{\kappa-\omega_{1}}
\end{aligned}
$$

Verificamos as condiçòes: 
(i) $C l_{B}\left(\pi_{B}\left(F_{0}\right)\right) \cap C l_{B}\left(\pi_{B}\left(F_{1}\right)\right) \neq \emptyset$ :

$\pi_{B}\left(F_{0}\right)=\pi_{B^{\prime}}\left(F_{0}^{B^{\prime}}\right) \times 2^{B-B^{\prime}}$ pois $F_{0}^{B^{\prime}} \subseteq 2^{\omega_{1}}$.

De igual forma : $\pi_{B}\left(F_{1}\right)=\pi_{B^{\prime}}\left(F_{1}^{B^{\prime}}\right) \times 2^{B-B^{\prime}}$

Então,

$$
\begin{aligned}
& C l_{B}\left(\pi_{B}\left(F_{0}\right)\right) \cap C l_{B}\left(\pi_{B}\left(F_{1}\right)\right)=C l_{B^{\prime}}\left(F_{0}^{B^{\prime}}\right) \times 2^{B-B^{\prime}} \cap C l_{B^{\prime}}\left(F_{1}^{B^{\prime}}\right) \times 2^{B-B^{\prime}}= \\
& =\left[C l_{B^{\prime}}\left(F_{0}^{B^{\prime}}\right) \cap C l_{B^{\prime}}\left(F_{1}^{B^{\prime}}\right)\right] \times 2^{B-B^{\prime}} \neq \emptyset \text { pois } C l_{B^{\prime}}\left(F_{0}^{B^{\prime}}\right) \cap C l_{B^{\prime}}\left(F_{1}^{B^{\prime}}\right) \neq \emptyset
\end{aligned}
$$

(ii) Seja $\alpha=\alpha_{B^{\prime}}$. Temos que provar que $\pi_{\{\alpha\}}\left(\pi_{B}^{-1}\left(\pi_{B}\left(F_{i}\right)\right) \cap H\right)=\{i\}$ para $i=0,1$. $\alpha_{B^{\prime}} \in\left(\omega_{1}-B^{\prime}\right)$ e $B^{\prime}=B \cap \omega_{1}$ portanto $\alpha_{B^{\prime}} \in\left(\omega_{1}-B\right) \subset(\kappa-B)$. $\pi_{B}\left(F_{i}\right)=\pi_{B^{\prime}}^{\omega_{1}}\left(F_{i}^{B^{\prime}}\right) \times 2^{B-B^{\prime}}$, então $\pi_{B}^{-1}\left(\pi_{B}\left(F_{i}\right)\right)=\left(\pi_{B^{\prime}}^{\omega_{1}}\right)^{-1}\left(\pi_{B^{\prime}}^{\omega_{1}}\left(F_{i}^{B^{\prime}}\right)\right) \times 2^{\kappa-\omega_{1}}$ $\operatorname{Logo} \pi_{B}^{-1}\left(\pi_{B}\left(F_{i}\right)\right) \cap H=\left[\left(\pi_{B^{\prime}}^{\omega_{1}}\right)^{-1}\left(\pi_{B^{\prime}}^{\omega_{1}}\left(F_{i}^{B^{\prime}}\right)\right) \times 2^{\kappa-\omega_{1}}\right] \cap\left(F \times 2^{\kappa-\omega_{1}}\right)=$ $=\left(\pi_{B^{\prime}}^{\omega_{1}}\right)^{-1}\left(\pi_{B^{\prime}}^{\omega_{1}}\left(F_{i}^{B^{\prime}}\right)\right) \cap F \times 2^{\kappa-\omega_{1}}$

portanto $\pi_{\{\alpha\}}\left(\left(\pi_{B^{\prime}}^{\omega_{1}}\right)^{-1}\left(\pi_{B^{\prime}}^{\omega_{1}}\left(F_{i}^{B^{\prime}}\right)\right) \cap F \times 2^{\kappa-\omega_{1}}\right)=\pi_{\{\alpha\}}^{\omega_{1}}\left(\left(\pi_{B^{\prime}}^{\omega_{1}}\right)^{-1}\left(\pi_{B^{\prime}}^{\omega_{1}}\left(F_{i}^{B^{\prime}}\right)\right) \cap F=\{i\}\right.$

Os seguintes teoremas são consequência imediata dos teoremas (3.10) e (3.11) e do lema (3.12).

Teorema 3.13 Para cada cardinal $\kappa$ não enumerável, existe um conjunto splitting $F \subset 2^{\kappa}$.

Teorema 3.14 Seja $\kappa$ um cardinal não enumerável. Suponhamos que para cada $\alpha<\kappa, X_{\alpha}^{\prime}$ é um subespaço compacto convexo com mais de um ponto, de um espaço linear normado. Então $\prod_{\alpha<\kappa} X_{\alpha}$ não tem CIP. 


\section{Capítulo 4}

\section{Conjuntos de pontos fixos de}

\section{homeomorfismos : dois exemplos}

O objetivo deste capítulo é provar que o cubo de Hilbert $Q$ e o cubo de Cantor $D^{\tau}$ onde $\tau$ é um cardinal infinito, tem CIPH.

\subsection{CIPH no cubo de Hilbert}

Lema 4.1 Todo subconjunto aberto de um espaço com CIPH, tem CIPH.

\section{Demonstração:}

Seja $X$ um espaço com CIPH, e seja $U$ um subconjunto aberto de $X$. Vamos provar que $U$ tem CIPH.

Sejam $A$ um subconjunto fechado de $U$, e $B=\mathrm{X}-U$. Como $X-(A \cup B)=U-A$ que é aberto em $U$, temos que $X-(A \cup B)$ é aberto em $X$ e portanto $A \cup B$ é um subconjunto fechado de $X$. Por hipótese, existe um homeomorfismo $h: X \longrightarrow X$ tal que $f i x(h)=A \cup B$

Seja $f=\left.h\right|_{U^{\prime}}, f: U \longrightarrow U$ e $f(U)=U$ pois $f(x)=x \quad \forall x \in X-U$ e $h$ é bijetora. 
Entào $f^{-1}=\left.h^{-1}\right|_{U}$ e $f$ é um homeomorfismo.

$f i x(f)=f i x(h) \cap U=$. A. como queríamos.

Teorema 4.2 Se X' $X$ é um espaço métrico e A é um subconjunto fechado não vazio de $X \times S^{1} \quad(X \times \mathbb{R})$, então existe um autohomeomorfismo de $X \times S^{1} \quad(X \times \mathbb{R})$ com A como conjunto de pontos fixos (i.e. $X \times S^{1}$ e $X \times \mathbb{R}$ tem CIPH).

\section{Demonstração:}

Sejam $d_{1}$ uma métrica limitada por 1 para $X$, e $d_{2}$ a métrica do comprimento do arco em $S^{1}$. Definimos em $\mathrm{X} \times S^{1}$ a seguinte métrica: se $u=\left(u_{1}, u_{2}\right)$ e $v=\left(v_{1}, v_{2}\right)$ são pontos de $\mathrm{X} \times S^{11}$ entào

$$
[d(u, v)]^{2}=\left[d_{1}\left(u_{1}, v_{1}\right)\right]^{2}+\left[d_{2}\left(u_{2}, v_{2}\right)\right]^{2}
$$

Se $z=(x, p) \in X \times S^{1}$ e $q \in S^{1}$, consideramos a seguinte $S^{1}$-ação em $X \times S^{1}$ : $\tilde{z} q=(x, p q)$ onde $p q$ denota o produto de números complexos.

Dado $A \subset X \times S^{1}$, fechado não vazio, definimos $f: X \times S^{1} \longrightarrow X \times S^{1}$ por :

$$
f(z)=z e^{i a(z)} \quad \text { onde } a(z)=1 / 2 \cdot d(z, A)
$$

$f$ é contínua:

Se $z=(x, p) \in X \times S^{1} \Rightarrow f(z)=\left(x, p e^{i a(z)}\right) \Rightarrow \pi_{X} f(z)=\pi_{X}(z) \quad \mathrm{e}$ $\pi_{S^{1}} f(z)=p e^{i a(z)}$. Como $d$ é uma distância, $a$ é contínua, portanto $\pi_{S^{1}} f$ é também contínua. $\pi_{X} f$ é contínua por ser uma projeção. Logo a função $f$ é contínua.

A é o conjunto de pontos fixos de $f$ :

Se $z \in A, a(z)=0$ e portanto $f(z)=z$.

Se $z=(x, p) \notin A$, temos que $0<d(z, A)<2 \pi$ (pois $d_{1} \leq 1$ e $d_{2} \leq \pi$ ). Daí $0<a(z)<2 \pi$. Entào $p e^{i a(z)} \neq p \quad$ e $\quad f(z) \neq z$.

$f$ é injetora:

Suponhamos $f(y)=f(z)$, entào por definição de $f, \pi_{X}(y)=\pi_{X}(z)$. Logo se 
$y=(x, p)$, temos que $z=(x, q)$ e para algum número real $t, p=q e^{i t} \operatorname{com} d(y, z)=$ $|t| \leq \pi$. Portanto se $q=e^{i \theta}, p=e^{i(\theta+t)}$.

$f(y)=f(z) \Rightarrow p e^{i a(y)}=q e^{i a(z)} \Rightarrow e^{i(\theta+t+a(y))}=e^{i(\theta+a(z))} \Rightarrow e^{i(t+a(y))}=e^{i a(z)} \Rightarrow$ $t+a(y)-a(z)=2 \pi n$ para algum inteiro $n$.

Aplicando a propriedade triangular a $y, z, A$ obtemos que $|t| \geq 2|a(y)-a(z)|$ e como $|t| \leq \pi$ temos que $|a(y)-a(z)| \leq \pi / 2$.

Resumindo :

$$
\begin{aligned}
& t+a(y)-a(z)=2 \pi n \\
& |t| \leq \pi \\
& |a(y)-a(z)| \leq \pi / 2
\end{aligned}
$$

então $t+a(y)-a(z)=0$ e como $|t| \geq 2|a(y)-a(z)|$ temos que $t=0$ e logo que $y=z$.

$f$ é uma função fechada:

Seja $B$ um subconjunto fechado de $X \times S^{1}$. e seja $(u, v) \in \overline{f(B)}$. Existe uma sequência $\left(\left(x_{n}, y_{n}\right)\right)_{n \in \omega}$ de pontos de $B$ tal que $\lim _{n \rightarrow \infty} f\left(x_{n}, y_{n}\right)=(u, v)$. Por defnição de $f$, $\lim x_{n}=u$. Vamos provar que a sequência $\left(y_{n}\right)_{n \in \omega}$ é convergente.

Suponhamos que $\left(y_{n}\right)_{n \in \omega}$ não converge. Como $S^{1}$ é métrico compacto e $\left(y_{n}\right)_{n \in \omega} \subset S^{1}$, esta suposição implica que $\left(y_{n}\right)_{n \in \omega}$ possui mais de um ponto de acumulação. Sejam $p$ e $q$ pontos de acumulação distintos de $\left(y_{n}\right)_{n \in \omega}$. Consideramos $\left(y_{n_{k}}\right)_{k \in \omega}$ e $\left(y_{n_{\jmath}}\right)_{j \in \omega}$ duas subsequências de $\left(y_{n}\right)_{n \in \omega}$ tais que $\quad \lim _{k \rightarrow \infty} y_{n_{k}}=p$ e $\lim _{j \rightarrow \infty} y_{n_{j}}=q$.

Temos então que

$$
\begin{aligned}
\lim _{k \rightarrow \infty} f\left(x_{n_{k}}, y_{n_{k}}\right) & =f(u, p) \\
\mathrm{e} & \\
\lim _{j \rightarrow \infty} f\left(x_{n_{j}}, y_{n_{j}}\right) & =f(u, q)
\end{aligned}
$$

Como $f$ é injetora e $p \neq q, f(u, p) \neq f(u, q)$. Chegamos portanto a uma contradição com $\lim f\left(x_{n}, y_{n}\right)=(u, v)$. Segue que a sequência $\left(y_{n}\right)_{n \in \omega}$ é convergente.

Seja $q=\lim _{n}$, então $\lim _{n \rightarrow \infty}\left(x_{n}, y_{n}\right)=(u, q) \in B$. Como $f$ é contínua $f(u, q)=\lim f\left(x_{n}, y_{n}\right)=(u, v), \operatorname{logo}(u, v) \in f(B)$. 


\section{$f$ é sobrejetora:}

lamos provar que $f\left(\{x\} \times S^{1}\right)=\{x\} \times S^{1} \forall x \in X^{\prime}$

Se $f\left(\{x\} \times S^{1}\right) \subsetneq\{x\} \times S^{1}$ para cada $x$, então $f\left(\{x\} \times S^{1}\right)$ é um arco de $S^{1}$. Mas como $\left.f\right|_{\{x\} \times S^{1}}$ é homeomorfismo na imagem teriamos $\{x\} \times S^{1}$ homeomorfo um arco de $S^{1}$ o que não é possivel.

Falta ver que $X \times \mathbb{R}$ tem CIPH. Sabemos que $X \times \mathbb{R}$ é homeomorfo a $X \times\left(S^{1}-\{1\}\right) \quad$ subconjunto aberto de $X \times S^{1}$. Segue pelo lema (4.1), $X \times \mathbb{R}$ tem CIPH.

Teorema 4.3 O cubo de Hilbert $Q$ tem CIPH.

\section{Demonstração:}

lamos identificar os pontos do plano $\mathbb{R}^{2}$ com números complexos, e usaremos $\mid$. | para denotar a norma usual em $\mathbb{C}$.

Para cada $n \in \omega$ seja $D_{n}=\left\{z \in \mathbb{C}:|z| \leq 1 / 2^{n}\right\}$. O cubo de Hilbert será representado por

$$
Q=\prod_{n=1}^{\infty} D_{n}
$$

e a métrica considerada em $Q$ é a seguinte: sejam $x=\left(x_{n}\right)_{n \in \omega} \in Q$ e $y=\left(y_{n}\right)_{n \in \omega} \in Q$ $\operatorname{com} x_{n}, y_{n} \in D_{n}$, então

$$
[d(x, y)]^{2}=\sum_{n=1}^{\infty}\left|x_{n}-y_{n}\right|^{2}
$$

Seja $A$ um subconjunto fechado não vazio de $Q$. Como $Q$ é homogêneo podemos assumir. sem perda de generalidade que $0=(0,0, \ldots) \in A$ Para cada $n \in \omega$ definimos a função $h_{n}: Q \longrightarrow Q$ como segue:

$$
\left(h_{n}(x)\right)_{j}= \begin{cases}x_{j} & \text { se } j \neq n \\ x_{n} e^{i a(x)} & \text { se } j=n \text { onde } a(x)=1 / 2 . d(x, A)\end{cases}
$$

Para cada $n \in \omega, h_{n}$ é uma funçào bijetora:

Fixado $n$. para cada $t$ tal que $0<t \leq 1 / 2^{n}$, temos que o conjunto 
$X_{t}=\left\{x \in Q:\left|x_{n}\right|=t\right\}$ é da forma $X \times S^{1}$.

Então para cada $0<t \leq 1 / 2^{n},\left.h_{n}\right|_{X_{t}}: X_{t} \longrightarrow X_{t}$ é um homeomorfismo (foi demonstrado no teorema anterior). Por definição de $h_{n}$ sabemos que $h_{n}(0)=0$. Logo $h_{n}$ é bijetora.

$h_{n}$ é contínua :

$$
\begin{aligned}
& \pi_{j} h_{n}(x)=\pi_{j}(x) \quad \forall j \neq n \forall x \in Q \Rightarrow \pi_{j} h_{n} \text { é contínua } \forall j \neq n \\
& \pi_{n} h_{n}(x)=x_{n} \cdot e^{i a(x)} \forall x \in Q \Rightarrow \pi_{n} h_{n} \text { é contínua }
\end{aligned}
$$

$h_{n}$ é fechada:

Seja $B$ um subconjunto fechado não vazio de $Q$. Então $B$ é compacto e $h_{n}(B)$ é fechado.

Provamos então que para cada $n \in \omega$ a função $h_{n}$ é um autohomeomorfismo de $Q$. Sejam agora $f_{n}=h_{n} \ldots h_{2} h_{1}$ para cada $n$, e $h=\lim _{n \rightarrow \infty} f_{n}$. Vamos mostrar que $h$ é um autohomeomorfismo de $Q$ e que $f i x(h)=A$. Para cada $n$ temos que

$$
d\left(f_{n+1}, f_{n}\right)=d\left(h_{n+1} h_{n} \ldots h_{1}, h_{n} \ldots h_{1}\right)=d\left(h_{n+1}, i d_{Q}\right)
$$

pois $h_{n} \ldots h_{1}$ é sobrejetora.

$$
d\left(h_{n+1}, i d_{Q}\right)=\sup _{x \in Q}\left\{\left|x_{n+1}\right| \cdot\left|e^{i a(x)}-1\right|\right\} \leq 1 / 2^{n}
$$

logo a sequência $\left(f_{n}\right)_{n \in \omega}$ é de Cauchy. Como $C(Q, Q)$ é completo (com a norma do supremo), existe $\lim f_{n}=h$. Mais ainda, $h$ é uma funçào contínua e sobrejetora. (lembramos que o conjunto das funções contínuas e sobrejetoras entre dois espaços compactos, é fechado no espaço das funções contínuas entre tais espaços [Mil\$s]). Sejam $x, y \in Q, x \neq y$. Suponhamos primeiro que $x, y \neq 0$. Seja $n_{0} \in \omega$ tal que $x_{n_{0}} \neq y_{n_{0}}$.

$h_{n_{0}}(x) \neq h_{n_{0}}(y):$

$x_{n_{0}} \in D_{n_{0}} \Rightarrow x_{n_{0}}=t e^{i \theta}$ com $0<t \leq 1 / 2^{n_{0}}$. De igual forma $y_{n_{0}}=s e^{i s}$ com $0<s \leq 1 / 2^{n_{0}}$. Temos entào que $t \neq s$ ou $\theta \neq \delta$. 
Se $t \neq s$ entào $h_{n_{0}}(x) \neq h_{n_{0}}(y)$ pois $\left.h_{n_{0}}\right|_{X_{t}}: X_{t} \longrightarrow X_{t}$.

Se $t=s$, como $\left.h_{n_{0}}\right|_{x_{t}}$ é um homeomorfismo. e $\theta \neq \delta, h_{n_{0}}(x) \neq h_{n_{0}}(y)$.

Por definição das funçòes $h_{n},\left(h_{m}\left(h_{n_{0}}(x)\right)\right)_{n_{0}}=\left(h_{n_{0}}(x)\right)_{n_{0}} \quad \forall m \neq n_{0} \mathrm{e}\left(h_{m}(x)\right)_{n_{0}}=$ $(x)_{n_{0}} \forall m \neq n_{0}, \quad \forall x \in Q$. Portanto $\left(f_{m}(x)\right)_{n_{0}}=\left(h_{n_{0}}(x)\right) \forall m>n_{0} \forall x \in Q$. Então $h_{n_{0}}(x) \neq h_{h_{0}}(y)$ implica $h(x) \neq h(y)$

Se $x=0, h(x)=0$. Nas como $y \neq 0$ existe $n_{0} \in \omega$ tal que $y_{n_{0}} \neq 0$. Logo $h_{n_{0}}(y) \neq 0$ e portanto $h(y) \neq 0$.

$f i x(h)=A$ :

Se $x \in A \Rightarrow a(x)=0 \Rightarrow h_{n}(x)=x \quad \forall n \in \omega \Rightarrow h(x)=x$

Se $x \notin A$, então $x_{n} \neq 0$ para algum $n, \operatorname{logo}(h(x))_{n} \neq x_{n}$ (lembramos que como $x \notin A, 0<a(x)<2 \pi)$.

\subsection{CIPH no cubo de Cantor}

Se $X$ é um espaço topológico, denotamos por $2^{X}$ o conjunto de todos os subconjuntos fechados não vazios de $X$. Em $2^{X}$ consideramos a topologia de Vietoris, cujos abertos básicos sào da forma:

$$
\left\langle U_{1}, \ldots, U_{k}\right\rangle=\left\{F \in 2^{X}: F \subset \bigcup_{i=1}^{n} U_{i} \quad \text { e } \quad F \cap U_{i} \neq \emptyset \quad \text { para } \quad i=1, \ldots, k\right\}
$$

onde $U_{1}, \ldots, U_{k}$ sào abertos de $X$.

Se $X$ é um espaço métrico compacto, consideramos em $2^{X}$ a métrica de Hausdorff $\left(d_{H}\right)$, definida por:

para $E, F \in 2^{x} \cdot d_{H}(E, F)=\inf \{\varepsilon>0: E \subseteq B(F, \Xi)$ e $F \subseteq B(E, \varepsilon)\}$

onde $B(F, \varepsilon)=\left\{x \in X^{\prime}: d(x, F)<\varepsilon\right\}$. A topologia induzida por $d_{H}$ em $2^{X}$ coincide com a topologia de Vietoris. Se $X^{\prime}$ é métrico compacto então $2^{X}$ é compacto (4.i de [Milss]). 


\section{Notações:}

* C denotará o conjunto de Cantor, considerado como subespaço do intervalo real $I$ e $d(x, y)$ denotará $|x-y|$ em $C$.

* $A C$ denotará o subespaço de $2^{C}$ dos subconjuntos fechados de $C$ que são densos em si mesmos (nos referiremos a estes conjuntos como subconjuntos completos de $C$ ).

* Seja $H$ subconjunto fechado de $C$. Denotamos:

$$
\begin{aligned}
& D_{0}(H)=\left\{x \in H: x \leq \frac{\min (H)+\max (H)}{2}\right\} \\
& D_{1}(H)=\left\{x \in H: x \geq \frac{\min (H)+\max (H)}{2}\right\}
\end{aligned}
$$

Obsevamos que como $H \subset C,(\min (H)+\max (H)) / 2 \notin H, \operatorname{logo} D_{0}(H) \cap D_{1}(H)=\emptyset$.

Lema 4.4 A relação $D_{i}(K)$ para $i=\{0,1\}$ é contínua na métrica de Hausdorff i.e. para cada $K \subseteq C$ fechado, dado $\varepsilon>0$ existe $\delta>0$ tal que para cada $G \in 2^{C}$ com $d_{H}\left(K^{\prime}, G^{\prime}\right)<\delta$ temos $d_{H}\left(D_{i}\left(K^{\prime}\right), D_{i}(G)\right)<\varepsilon$.

\section{Demonstração:}

Sejam $\quad a_{1}=\min \left(K^{\prime}\right), \quad d_{1}=\max \left(K^{\prime}\right), \quad c_{1}=\min \left(D_{1}\left(K^{\circ}\right)\right), \quad b_{1}=\max \left(D_{0}\left(K^{\circ}\right)\right)$, $M_{1}=\left(a_{1}+d_{1}\right) / 2$.

$M_{1} \notin C$ portanto existe $\gamma>0$ tal que $\gamma<\min \left\{d\left(b_{1}, M_{1}\right), d\left(M_{1}, c_{1}\right)\right\}$.

Dado $\varepsilon>0$ seja $\delta=\min \{\gamma / 3, \varepsilon\}$ e seja $G \subset C$ fechado, tal que $d_{H}(K, G)<\delta$. Definimos $a_{2}, b_{2}, c_{2}, d_{2}, M_{2}$ para $G$, em forma análoga a $a_{1}, \ldots, M_{1}$. Como

$$
\left|M_{1}-M_{2}\right|=\left|\left(a_{1}-a_{2}+d_{1}-d_{2}\right) / 2\right| \leq\left|a_{1}-a_{2}\right| / 2+\left|d_{1}-d_{2}\right| / 2 \text { e } d_{H}(K, G)<\delta
$$

temos que $\left|M_{1}-M_{2}\right|<\delta$. Como $d\left(b_{1}, M_{1}\right)>3 \delta$ temos que $d\left(b_{1}, M_{2}\right)>\delta \quad$ e $b_{1}<M_{2}$ pois $b_{1}<M_{1}$. Logo $d\left(b_{1}, c_{2}\right)>\delta$ e portanto $B\left(D_{1}(G), \delta\right) \cap D_{0}\left(K^{\prime}\right)=\emptyset$. De $d_{H}\left(K^{\prime} \cdot G\right)<\delta$ segue que $D_{0}(K) \subset B\left(D_{0}(G), \delta\right) \cup B\left(D_{1}(G), \delta\right)$. Logo $D_{0}\left(K^{\prime}\right) \subset$ $B\left(D_{0}(G), \delta\right)$.

De igual forma $d\left(b_{1}, c_{2}\right)<\delta$ implica que $B\left(D_{0}\left(K^{*}\right), \delta\right) \cap D_{1}(G)=\emptyset$, portanto 
$D_{1}(G) \subset B\left(D_{1}(K), \delta\right)$.

Trabalhando em forma análoga com $d\left(c_{1}, M_{1}\right)>3 \delta$ obtemos $D_{1}\left(K^{\prime}\right) \subset B\left(D_{1}(G), \delta\right)$ e $D_{0}(G) \subset B\left(D_{0}(K), \delta\right)$.

Resumindo temos $d_{H}\left(D_{i}(K), D_{i}(G)\right)<\varepsilon$ para $i=0,1$.

Lema 4.5 Existe uma função contínua $f: C \times A C \longrightarrow C$ sobrejetora, tal que:

(1) $f(C \times\{K\})=K$ para cada $K \in A C \quad e$

(2) $\left.f\right|_{C \times\{K\}}$ é um homeomorfismo para cada $K \in A C$.

\section{Demonstração:}

Vamos primeiro construir a função $f$.

Observamos que se $H$ é um subconjunto completo de $C$, então $D_{i}(K)$ também o é. Para cada conjunto $\{i(1), \ldots, i(n)\}$ onde $i(j) \in\{0,1\}$ para $j=1, \ldots, n$, definimos o conjunto $D_{i(1) \ldots i(n)}(K)$ como segue:

$$
D_{i(1) \ldots i(n)}\left(K^{i}\right)=D_{i(n)}\left(D_{i(1) \ldots i(n-1)}(K)\right)
$$

afirmação 1: Os conjuntos $D_{i(1) \ldots i(n)}(K)$ formam uma base para $K$, e cada ponto $x \in K^{\prime}$ determina uma sequência $J(x, K)=\{i(j)\}_{j=1}^{\infty}$ com $i(j) \in\{0,1\} \forall j$, tal que $D_{i(1)}(K) \supset D_{i(1) i(2)}(K) \supset \ldots \ni x$.

prova:

(i) $D_{i}(K)$ é um aberto de $K$ :

$$
\begin{aligned}
& D_{0}\left(K^{\circ}\right)=\left[0, \frac{\max \left(K^{\prime}\right)+\min \left(K^{\prime}\right)}{2}\right) \cap K^{\prime} \\
& D_{1}\left(K^{\circ}\right)=\left(\frac{\max \left(K^{\circ}\right)+\min \left(K^{\prime}\right)}{2}, 1\right] \cap K^{\prime}
\end{aligned}
$$

(ii) Seja $x \in K$ e seja $U$ uma vizinhança aberta de $x$ em $K$.

Se $x \in D_{0}(K)$ então $i(1)=0$; se $x \in D_{1}(K)$ então $i(1)=1$. Portanto $x \in D_{i(1)}(K)$. Se $x \in D_{0}\left(D_{i(1)}\left(K^{\prime}\right)\right)$, então $i(2)=0$, se não $i(2)=1$. ou seja $x \in D_{i(1) i(2)}\left(K^{\prime}\right)$. 
Assim $x$ determina uma sequência $\{i(j)\}_{j=1}^{\infty}$. Como $\operatorname{diam}\left(D_{i(1) \ldots i(n)}(K)\right) \leq 1 / 2^{n}$ temos que $\{x\}=\bigcap_{n=1}^{\infty} D_{i(1) \ldots i(n)}(K)$, e existe $n_{0} \in \omega$ tal que $D_{i(1) \ldots i\left(n_{0}\right)}(K) \subset U$.

afirmaçào 2: Cada sequência $J=\{i(j)\}_{j=1}^{\infty} \operatorname{com} i(j) \in\{0,1\}$, determina um ponto $x\left(J, K^{\circ}\right)$ satisfazendo a igualdade $J\left(x\left(J, K^{\circ}\right)\right)=J$.

prova:

Seja $J=\{i(j)\}_{j=1}^{\infty}$. Para cada $n \in \omega, D_{i(1) \ldots i(n)}\left(K^{\prime}\right)$ é um subconjunto completo de $C$, e portanto $D_{i(1) \ldots i(n) i(n+1)}(K) \neq \emptyset$. Temos também que

$$
K \supset D_{i(1)}(K) \supset D_{i(1) i(2)}(K) \supset \ldots
$$

Logo como $K$ é compacto, $\bigcap_{n=1}^{\infty} D_{i(1) \ldots i(n)}(K) \neq \emptyset$. E de novo usando o diâmetro de $D_{i(1) \ldots i(n)}\left(K^{i}\right)$ temos que esta intersecção tem um único ponto.

Definimos a funçào $f: C \times A C \longrightarrow C$ como segue:

$$
f\left(x, K^{\prime}\right)=x\left(J(x, C), K^{\prime}\right)
$$

$f$ é contínua :

Sejam $\left(x, K^{\circ}\right) \in C \times A C$ e $\left(x_{n}, K_{n}\right)_{n \in \omega}$ uma sequência em $C \times A C$ convergindo para $(x, K)$. Temos então que $\lim _{n \rightarrow \infty} d\left(x_{n}, x\right)=0$ e $\lim _{n \rightarrow \infty} d_{H}\left(\kappa_{n}, K^{\prime}\right)=0$.

Devemos provar que dado $\varepsilon>0$, existe $n_{0} \in \omega$ tal que $d\left(f\left(x_{n}, K_{n}\right), f\left(x, K^{\prime}\right)\right)<\varepsilon$ $\forall n \geq n_{0}$.

Vamos usar $y_{n}$ como notaçào para $f\left(x_{n}, K_{n}\right)$ e $y$ para $f(x, K)$.

Então $y_{n}=x\left(J\left(x_{n}, C\right), K_{n}\right)$ e $y=x\left(J(x, C), K^{\prime}\right)$. Como $\lim _{n \rightarrow \infty} d_{H}\left(K_{n}, K^{\prime}\right)=0$, pelo lema (4.4) temos que $\lim _{n \rightarrow \infty} d_{H}\left(D_{i(1) \ldots i(m)}\left(K_{n}\right), D_{i(1) \ldots i(m)}\left(K^{\prime}\right)\right)=0$ para toda sequência $\{i(j)\}$ com $i(j) \in\{0,1\}$, para todo $m \in \omega$.

Seja $m$ tal que $1 / 2^{m}<\varepsilon / 2$. Como $\lim _{n \rightarrow \infty} d\left(x_{n}, x\right)=0$ existe $n_{1} \in \omega$ tal que $\forall n \geq n_{1}$ $J\left(x_{n}, C\right)$ e $J(x, C)$ coincidem nos $m$ primeiros termos.

Sejam $i(1), \ldots, i(m)$ os primeiros $m$ termos de $J(x, C)$, e seja $n_{2} \in \omega$ tal que $\forall n \geq n_{2} \quad d_{H}\left(D_{i(1) \ldots i(m)}\left(K_{n}^{*}\right), D_{i(1) \ldots i(m)}\left(K^{*}\right)\right)<\varepsilon / 2$. Seja $n_{0}=\max \left\{n_{1}, n_{2}\right\}$. Como para cada $n \geq n_{0}, y_{n} \in D_{i(1) \ldots i(m)}\left(K_{n}\right)$ e $d_{H}\left(D_{i(1) \ldots i(m)}\left(K_{n}\right), D_{i(1) \ldots i(m)}\left(K^{\prime}\right)\right)<\varepsilon / 2$, 
temos que $d\left(y_{n}, D_{i(1) \ldots i(m)}\left(K^{\prime}\right)\right)<\varepsilon / 2$.

Como $\operatorname{diam}\left(D_{i(1) \ldots i(m)}\left(K^{\prime}\right)\right) \leq 1 / 2^{m}<\varepsilon / 2$, segue que $d\left(y_{n}, y\right)<\varepsilon \forall n \geq n_{0}$.

$f$ é sobrejetora:

Se $y \in C, f(y, C)=y$.

(1) $f\left(C \times\left\{K^{\prime}\right\}\right)=H$ para cada $K^{\prime} \in A C$ :

$f\left(C \times\left\{K^{\prime}\right\}\right) \subset K^{\prime}$ por definição de $f$.

Seja $y \in \Pi$. Existe $x \in C$ tal que $J(x, C)=J(y, K)$. Então $f(x, K)=y \mathrm{e}$ $K \subset f\left(C \times\left\{K^{\circ}\right\}\right)$.

(2) $\left.f\right|_{C \times\{K\}}$ é um homeomorfismo:

$\left.f\right|_{C \times\left\{K^{\prime}\right\}}: C \times\left\{K^{\circ}\right\} \longrightarrow K^{\prime}$, é sobrejetora por (1).

Se $x, x^{\prime} \in C, x \neq x^{\prime}$ entào $J(x, C) \neq J\left(x^{\prime}, C\right)$, portanto $x(J(x, C), K) \neq x\left(J\left(x^{\prime}, C\right), K^{\prime}\right)$

e $\left.f\right|_{C \times\{K\}}$ é injetora.

Definição 4.6 Sejam X e $Y$ espaços compactos Hausdorff. Uma função $\phi: X \longrightarrow Y$ é chamada uma d-aplicação se é sobrejetora, contínua, aberta e existe uma imersão de $X \in m C \times Y$ tal que $\phi=\left.\pi_{Y}\right|_{X}$, onde $\left.\pi_{Y}\right|_{X}$ é a restriçâo da projeção $\pi_{Y}: C \times Y \longrightarrow Y$ ao conjunto $X$.

Lema 4.7 Sejam $X$ e $Y$ espaços compactos Hausdorff. Se $\phi: X \longrightarrow Y$ é uma daplicação, e se $\pi_{C} \phi^{-1}(y)$ é completo para cada $y \in Y$, então existe um homeomorfismo $g: C \times Y \longrightarrow X$ tal que $\pi_{Y}=\phi g$.

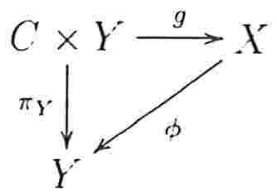

\section{Demonstração:}

Por definição de d-aplicação existe uma imersão de $X^{\prime}$ em $C \times Y$ tal que $\phi=\left.\pi_{Y}\right|_{X}$. 
Definimos $g: C \times Y \longrightarrow X$ como segue: $\quad$ se $(c, y) \in C \times Y$

$$
g(c, y)=\left(f\left(c, \pi_{C} \phi^{-1}(y)\right), y\right)
$$

onde $f: C \times A C \longrightarrow C$ é definida como no lema (4.5).

(i) $g(c, y) \in X^{\prime} \quad \forall(c, y) \in C \times Y$ pois $\phi^{-1}(y) \subset X \forall y \in Y$.

(ii) $g$ é injetora :

Sejam $(c, y),\left(c^{\prime}, y^{\prime}\right)$ dois pontos distintos de $C \times Y$.

Se $y \neq y^{\prime}$, entào $g(c, y) \neq g\left(c^{\prime}, y^{\prime}\right)$ por definição da função $g$.

Se $y=y^{\prime}$, entào $c \neq c^{\prime}$ e como $\left.f\right|_{C \times\left\{\pi_{C} \phi^{-1}(y)\right\}}$ é um homeomorfismo temos que $g(c, y) \neq g\left(c^{\prime}, y^{\prime}\right)$.

(iii) $g$ é sobrejetora :

Se $(c, y) \in X$, então $c \in \pi_{C} \phi^{-1}(y)$ pois $(c, y) \in \phi^{-1}(y)$. Usando mais uma vez o fato que $\left.f\right|_{C \times\left\{\pi_{C} \phi^{-1}(y)\right\}}$ é um homeomorfismo, temos que existe $c^{\prime} \in C$ tal que $f\left(c^{\prime}, \pi_{C} \phi^{-1}(y)\right)=c$. Logo $g\left(c^{\prime}, y\right)=(c, y)$.

(iv) $g$ é contínua :

Seja $(c, y) \in C \times Y$ arbitrário. Vamos mostrar que $g$ é contínua em $(c, y)$.

Seja $H=\pi_{C^{\circ} \varphi^{-1}}(y)$.

Dado $\varepsilon>0$ seja $V_{s} \times W$ uma vizinhança aberta de $g(c, y)$, onde $V_{\varepsilon}=B(c, \varepsilon) \cap C$.

A função $f: C \times A C \longrightarrow C$ é contínua, portanto existem vizinhanças $\left\langle U_{1}, \ldots, U_{m}\right\rangle$ de $H$ em $2^{C}$ e $U$ de $c$ em $C$ tais que

$$
\text { se } H_{1} \in\left\langle U_{1}, \ldots, U_{m}\right\rangle \text { e } c_{1} \in U \text {, então } d\left(f\left(c_{1}, H_{1}\right), f(c, H)\right)<\varepsilon \text {. }
$$

Por definiçào de $\left\langle U_{1}, \ldots, U_{m}\right\rangle$ temos: $H \subset \bigcup_{i=1}^{m} U_{i}$ e $H \cap U_{i} \neq \emptyset \quad \forall i=1, \ldots, m$. Por outro lado temos: $y \in W \Rightarrow \phi^{-1}(y) \subset \phi^{-1}(W) \Rightarrow H=\pi_{C} \phi^{-1}(y) \subset \pi_{C} \phi^{-1}(W)$. Portanto $U_{i} \times Y \cap \phi^{-1}(W) \neq \emptyset \forall i=1, \ldots, m$ e como $\phi$ é uma função contínua. $U_{i} \times Y \cap \phi^{-1}(W)$ é aberto.

Para cada $i \in\{1, \ldots, m\}$, seja $O_{i}=\phi\left(U_{i} \times Y \cap \phi^{-1}(W)\right)$. Como $\phi$ é uma funçào 
aberta, $O_{i}$ é um aberto $\forall i \in\{1, \ldots, m\}$. Alem disso $y \in O_{i} \forall i$ pois $y \in W$. Por outro lado $O_{0}=Y-\phi\left(X-\bigcup_{i=1}^{m} U_{i} \times Y\right)$ é aberto em $Y$ e $y \in O_{0}$ ( pois $\left.\phi^{-1}(y) \subset \bigcup_{i=1}^{m} U_{i} \times Y\right)$.

Seja $O=\bigcap_{i=0}^{m} O_{i}$, então $U \times(O \cap W)$ é uma vizinhança aberta de $(c, y)$. Se $\left(c_{1}, y_{1}\right) \in$ $U \times(O \cap W)$ então $\pi_{C} \phi^{-1}\left(y_{1}\right) \in\left\langle U_{1}, \ldots, U_{m}\right\rangle$ :

(1) $y_{1} \in O_{0} \Longrightarrow y_{1} \notin O\left(X-\bigcup_{i=1}^{m} U_{i} \times Y\right) \Longrightarrow \phi^{-1}\left(y_{1}\right) \cap\left(X-\bigcup_{i=1}^{m} U_{i} \times Y\right)=\emptyset$ $\Longrightarrow \phi^{-1}\left(y_{1}\right) \subset \mathrm{X} \cap\left(\bigcup_{i=1}^{m} U_{i} \times Y\right) \Longrightarrow \pi_{C} \phi^{-1}\left(y_{1}\right) \subset \bigcup_{i=1}^{m} U_{i}$.

(2) $y_{1} \in O_{i} \forall i=1, \ldots m \Longrightarrow y_{1} \in \phi\left(U_{i} \times Y \cap \phi^{-1}(W)\right) \forall i=1, \ldots, m \Longrightarrow$ $\phi^{-1}\left(y_{1}\right) \cap\left(U_{i} \times Y \cap \phi^{-1}(W)\right) \neq \emptyset \forall i=1, \ldots, m \Longrightarrow o^{-1}\left(y_{1}\right) \cap U_{i} \times Y \neq \emptyset \forall i=$ $1, \ldots, m$.

Chamando $H_{1}$ a $\pi_{C} \phi^{-1}\left(y_{1}\right)$, temos que : $d\left(f(c, H), f\left(c_{1}, H_{1}\right)\right)<\varepsilon$ i.e. $f\left(c_{1}, H_{1}\right) \in V_{\varepsilon}$, $y_{1} \in W$. Portanto $g\left(c_{1}, y_{1}\right)=\left(f\left(c_{1}, H_{1}\right), y_{1}\right) \in V_{\varepsilon} \times W$.

(v) $\pi_{Y}=\phi g:$

$\phi g(c, y)=\left.\pi_{Y}\right|_{X}(g(c, y))=\pi_{Y}(g(c, y))=y$.

Definição 4.8 Uma aplicação contínua $f: X^{r} \longrightarrow Y$ é regular se para cada subespaço fechado $F$ de $X$ existe um homeomorfismo $h: X \longrightarrow X$ tal que fix $(h)=F$ efh $=f$.

Observamos que o dominio de uma aplicação regular tem CIPH.

Teorema 4.9 Sejam $\tau \geq \omega$ um cardinal e $K$ um espaço métrico separável. Se para cada subespaço fechado $X^{\prime}$ de $K^{\omega}$ a projeção $\pi_{1}: X \times K^{\omega} \longrightarrow X$ é regular, então a projeção $\pi_{1}: X \times K^{\tau} \longrightarrow X$ é regular para, cada subespaço fechado $X$ de $\Lambda^{-\tau}$. Em particular $\mathrm{X} \times \mathrm{K}^{\top}$ tem CIPH, onde $\mathrm{X}$ é um subespaço fechado de $\mathrm{K}^{-\tau}$.

\section{Demonstração:}

A prova será feita por indução transfinita sobre $\tau$. No caso $\tau=\omega, \pi_{1}: X \times K^{\omega} \longrightarrow X^{\prime}$ é regular por hipótese. 
Suponhamos que o teorema foi provado para todo $\tau<\kappa$, onde $\omega<\kappa$, e consideramos o caso $\tau=\kappa$. Seja entào. $X^{r}$ um subespaço fechado de $K^{\tau}$.

Se $\tau=\omega_{\alpha+1}$ (i.e. se $\tau$ é um cardinal regular), entào $K^{\tau}$ pode ser representado como o limite inverso do sistema $\left\{\left(\Lambda^{\omega_{\alpha}}\right)^{\beta}, \pi_{\beta}^{\beta+1}, \beta<c f(\tau)\right\}$, onde $\pi_{\beta}^{\beta+1}:\left(K^{\omega_{\alpha}}\right)^{\beta+1} \longrightarrow\left(K^{\omega_{\alpha}}\right)^{\beta}$ denota a projeção $\left(K^{\omega_{\alpha}}\right)^{\beta} \times K^{\omega_{\alpha}} \longrightarrow\left(K^{\omega_{\alpha}}\right)^{\beta}$. Ou seja que se $k_{\beta+1} \in\left(K^{\omega_{\alpha}}\right)^{\beta+1}$, então $\pi_{\beta}^{\beta+1}\left(k_{\beta+1}\right)=\left.k_{\beta+1}\right|_{\beta}$.

Se $\tau$ é um cardinal singular, então $\tau=\sup \left\{\omega_{\alpha_{\beta}}: \beta<c f(\tau)\right\}$, onde $\omega \leq \omega_{\alpha_{\beta}}<\tau$ para cada $\beta<c f(\tau)$. Portanto $\Lambda^{*}$ pode ser representado como o limite inverso do sistema $\left\{K^{\omega_{n_{3}}} \cdot \pi_{\beta}^{\beta+1} \cdot \beta<c f(\tau)\right\}$ onde $\pi_{\beta}^{\beta+1}: K^{{ }^{-w_{\alpha_{\beta+1}}}} \longrightarrow K^{\omega_{\alpha_{\beta}}}$ denota a projeção no subproduto correspondente. Ou seja que se $k_{\beta+1} \in K^{-\omega_{\beta+1}}$, então $\pi_{\beta}^{\beta+1}\left(k_{\beta+1}\right)=$ $\left.k_{\beta+1}\right|_{\omega_{\alpha_{\beta}}}$.

Combinando ambos casos, podemos escrever $K^{\tau}=\stackrel{\leftarrow}{\mathrm{im}} S_{K}$, com

$S_{K}=\left\{K_{\beta}, \pi_{\beta}^{3+1}, \beta<c f(\tau)\right\}$ onde $K_{\beta}= \begin{cases}\left(K^{\omega_{\alpha}}\right)^{\beta} & \text { se } \tau=\omega_{\alpha+1} \\ K^{-\omega_{\alpha_{\beta}}} & \text { se } \tau=\sup \left\{\omega_{\alpha_{\beta}}: \beta<c f(\tau)\right\}\end{cases}$

Por $\pi_{\beta}$ denotamos a projeção de $K^{\tau}$ em $K_{\beta}$ i.e. se $k \in K^{\tau}$,

$$
\pi_{\beta}(k)= \begin{cases}\left.k\right|_{\beta} & \text { se } \tau=\omega_{\alpha+1} \\ \left.k\right|_{\omega_{\alpha_{\beta}}} & \text { se } \tau=\sup \left\{\omega_{\alpha_{\beta}}: \beta<c f(\tau)\right\}\end{cases}
$$

Como $X$ é um subespaço fechado de $K^{\tau}$, temos que $X$ coincide com o limite inverso do sistema $S_{X}=\left\{X_{\beta}, p_{\beta}^{\beta+1}, \beta<c f(\tau)\right\}$ onde $X_{\beta}=C l_{K_{\beta}} \pi_{\beta}(X)$ e $p_{\beta}^{\beta+1}=\left.\pi_{\beta}^{\beta+1}\right|_{X_{\beta+1}}$. Seja $S_{X} \times S_{K}=\left\{X_{\beta} \times K_{\beta}, p_{\beta}^{\beta+1} \times \pi_{\beta}^{\beta+1}, \beta<c f(\tau)\right\}$, observamos que $\stackrel{\leftarrow i m}{\lim } S_{X} \times S_{K}=X \times K^{\tau}$.

Seja $F$ um subconjunto fechado de $X \times K^{\tau}$. Para cada $\beta<c f(\tau), F_{\beta}=C l_{K_{\beta}}\left(p_{\beta} \times \pi_{\beta}\right)(F)$.

A projeção $X_{\beta} \times K_{\beta} \longrightarrow X_{\beta}$ será denotada por $\pi_{1}^{\beta}$.

Como $X_{0}$ é fechado em $K_{0}$, e $K_{0}$ é o produto de menos de $\tau$ cópias de $K$, por hipótese de indução temos que existe um homeomorfismo $h_{0}: X_{0} \times K_{0} \longrightarrow X_{0} \times K_{0}$ tal que $\pi_{1}^{0} h_{0}=\pi_{1}^{0}$ e fix $\left(h_{0}\right)=F_{0}$. 


\section{Afirmação:}

Para cada $\gamma<c f(\tau)$ existe um homeomorfismo $h_{\gamma}: X_{\gamma} \times \Lambda_{\gamma} \longrightarrow X_{\gamma} \times K_{\gamma}$ tal que:

(i) $\pi_{\gamma}^{\gamma} h_{\gamma}=\pi_{1}^{\gamma}$

(ii) $)_{\gamma} \operatorname{fix}\left(h_{\gamma}\right)=F_{\gamma}$

(iii) $)_{\gamma}\left(p_{\delta}^{\gamma} \times \pi_{\delta}^{\gamma}\right) h_{\gamma}=h_{\delta}\left(p_{\delta}^{\gamma} \times \pi_{\delta}^{\gamma}\right)$ para cada $\delta \leq \beta$

$(i v)_{\gamma} h_{\gamma}=\overleftarrow{\lim }\left\{h_{\delta}: \delta<\gamma\right\}$ para cada ordinal limite $\gamma<c f(\tau)$.

Seja então $h$ o homeomorfismo definido por:

$$
h=\overleftarrow{\lim }\left\{h_{\beta}: \beta<c f(\tau)\right\}: X \times K^{\tau} \longrightarrow X^{r} \times K^{\tau}
$$

$h$ preserva a primeira coordenada e $f i x(h)=F$. Como queríamos.

\section{Demonstração da afirmação:}

Suponhamos que para cada $\beta<\gamma$, onde $\gamma<c f(\tau)$, já foram construidos homeomorfismos $h_{\beta}: X_{\beta} \times K_{\beta} \longrightarrow X_{\beta} \times K_{\beta}$ tais que :

(i) $\pi_{1}^{\beta} h_{\beta}=\pi_{1}^{\beta}$ para cada $\beta<\gamma$

$(\text { ii })_{\beta}$ fix $\left(h_{\beta}\right)=F_{\beta}$ para cada $\beta<\gamma$

$(\text { iiii })_{\beta}\left(p_{S}^{\beta} \times \pi_{\delta}^{\beta}\right) h_{\beta}=h_{\delta}\left(p_{\delta}^{\beta} \times \pi_{\delta}^{\beta}\right)$ para cada $\operatorname{par} \delta, \beta \operatorname{com} \delta \leq \beta<\gamma$

(iv) $h_{\beta}=\overleftarrow{\lim }\left\{h_{\delta}: \delta<\beta\right\}$ para cada ordinal limite $\beta<\gamma$.

Vamos agora definir o homeomorfismo $h_{\gamma}: X_{\gamma} \times K_{\gamma} \longrightarrow X_{\gamma} \times K_{\gamma}$.

* Se $\gamma$ é um ordinal limite, definimos $h_{\gamma}=\overleftarrow{\lim }\left\{h_{\beta}: \beta<\gamma\right\}$. Por definição de limite inverso para funções temos:

(iii) $)_{\gamma}\left(p_{\delta}^{\gamma} \times \pi_{\delta}^{\gamma}\right) h_{\gamma}=h_{\delta}\left(p_{\delta}^{\gamma} \times \pi_{\delta}^{\gamma}\right)$ para cada $\delta<\gamma$.

Para provar $(i)_{\gamma} \pi_{1}^{\gamma} h_{\gamma}=\pi_{1}^{\gamma}$, seja $\left(x_{\gamma}, k_{\gamma}\right) \in X_{\gamma} \times K_{\gamma}$. Temos que :

$$
\begin{aligned}
p_{\delta}^{\gamma}\left(\pi_{1}^{\gamma} h_{\gamma}\left(x_{\gamma}, k_{\gamma}\right)\right) & =\pi_{1}^{\delta}\left(p_{\delta}^{\gamma} \times \pi_{\delta}^{\gamma}\right) h_{\gamma}\left(x_{\gamma}, k_{\gamma}\right) \stackrel{(i i i)_{\gamma}}{=} \pi_{1}^{\delta} h_{\delta}\left(p_{\delta}^{\gamma} \times \pi_{\delta}^{\gamma}\right)\left(x_{\gamma}, k_{\gamma}\right) \\
& \stackrel{(i)_{\delta}}{=} \pi_{1}^{\delta}\left(p_{\delta}^{\gamma} \times \pi_{\delta}^{\gamma}\right)\left(x_{\gamma}, k_{\gamma}\right)=p_{\delta}^{\gamma}\left(x_{\gamma}\right) \quad \forall \delta<\gamma
\end{aligned}
$$

Portanto $\pi_{1}^{\gamma} h_{\gamma}\left(x_{\gamma}, k_{\gamma}\right)=\pi_{1}^{\gamma}\left(x_{\gamma}, k_{\gamma}\right)$ como queríamos. 
Falta provar $(\text { ii })_{\gamma}$ fix $\left(h_{\gamma}\right)=F_{\gamma}$ :

(1) $F_{\gamma} \subset$ fix $\left(h_{\gamma}\right)$

$F_{\gamma}=C l_{K_{\gamma}}\left(p_{\gamma} \times \pi_{\gamma}\right)(F)$. Como $h_{\gamma}$ é contínua, basta mostrar que

$\left(p_{\gamma} \times \pi_{\gamma}\right)(F) \subset$ fix $\left(h_{\gamma}\right)$. Seja $\left(x_{\gamma}, k_{\gamma}\right) \in\left(p_{\gamma} \times \pi_{\gamma}\right)(F)$. Por $(\text { iiii })_{\gamma}$ temos que

$$
\left(p_{\delta}^{\gamma} \times \pi_{\delta}^{\gamma}\right) h_{\gamma}\left(x_{\gamma}, k_{\gamma}\right)=h_{\delta}\left(p_{\delta}^{\gamma} \times \pi_{\delta}^{\gamma}\right)\left(x_{\gamma}, k_{\gamma}\right) \quad \forall \delta<\gamma
$$

mas como $\left(p_{\delta}^{\gamma} \times \pi_{\delta}^{\gamma}\right)\left(x_{\gamma}, k_{\gamma}\right) \in F_{\delta}$ temos também

$$
h_{\delta}\left(p_{\delta}^{\gamma} \times \pi_{\delta}^{\gamma}\right)\left(x_{\gamma}, k_{\gamma}\right)=\left(p_{\delta}^{\gamma} \times \pi_{\delta}^{\gamma}\right)\left(x_{\gamma}, k_{\gamma}\right) \quad \forall \delta<\gamma
$$

$\operatorname{Logo} h_{\gamma}\left(x_{\gamma}, k_{\gamma}\right)=\left(x_{\gamma}, k_{\gamma}\right)$.

(2) fix $\left(h_{\gamma}\right) \subset F_{\gamma}$ :

Seja $\left(x_{\gamma}, k_{\gamma}\right) \in$ fix $\left(h_{\gamma}\right)$, então $h_{\delta}\left(p_{\delta}^{\gamma} \times \pi_{\delta}^{\gamma}\right)\left(x_{\gamma}, k_{\gamma}\right) \in f i x\left(h_{\delta}\right) \forall \delta<\gamma$. Como $F_{\delta}=f i x\left(h_{\delta}\right) \forall \delta<\gamma$, temos que $p_{\delta}^{\gamma} \times \pi_{\delta}^{\gamma}\left(x_{\gamma}, k_{\gamma}\right) \in F_{\delta}=\overline{p_{\delta}^{\gamma} \times \pi_{\delta}^{\gamma}\left(F_{\gamma}\right)} \forall \delta<\gamma$.

Portanto $\left(x_{\gamma}, k_{\gamma}\right) \in F_{\gamma}$.

* Se $\gamma$ é ordinal sucessor i.e $\gamma=\beta+1$, procedemos como segue. Consideramos o conjunto

$$
Z_{\beta}=\left\{\left(x_{\beta}, k_{\beta}, x_{\beta+1}\right) \in \mathrm{X}_{\beta} \times K_{\beta} \times X_{\beta+1}: x_{\beta}=p_{\beta}^{\beta+1}\left(x_{\beta+1}\right)\right\}
$$

e as seguintes funções:

$$
\begin{aligned}
l= & \left(p_{\beta}^{\beta+1} \times \pi_{\beta}^{\beta+1}\right) \Delta \pi_{1}^{\beta+1} \\
& \varphi: Z_{\beta} \longrightarrow X_{\beta} \times K_{\beta}^{\prime} \text { e } \psi: Z_{\beta} \longrightarrow X_{\beta+1} \text { as correspondentes projeções }
\end{aligned}
$$

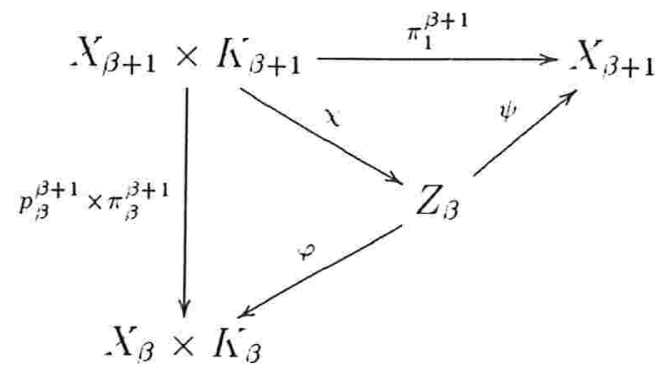


- Se $\tau=\sup \left\{\omega_{\alpha_{\beta}}: \beta<c f(\tau)\right\}$, para cada ponto $\left(x_{\beta}, k_{\beta}, x_{\beta+1}\right) \in Z_{\beta}$, temos que $x^{-1}\left(x_{\beta}, k_{3}, x_{\beta+1}\right)=\left\{\left(x_{\beta+1}, \hat{k}_{\beta+1}\right) \in X_{\beta+1} \times \kappa_{\beta+1}:\left.\hat{k}_{\beta+1}\right|_{\omega_{\alpha_{\beta}}}=k_{\beta}\right\} \approx K^{\lambda}$ onde $\lambda=\omega_{\alpha_{\beta+1}}-\omega_{\alpha_{\beta}}$. Definimos a funçào $j: X_{\beta+1} \times K_{\beta+1} \longrightarrow Z_{\beta} \times K^{\lambda}$ por :

$$
j\left(x_{\beta+1}, k_{\beta+1}\right)=\left(\left(\left.x_{\beta+1}\right|_{\omega_{\alpha_{\beta}}},\left.k_{\beta+1}\right|_{\omega_{\alpha_{\beta}}}, x_{\beta+1}\right),\left.k_{\beta+1}\right|_{\lambda}\right)
$$

$j$ é um homeomorfismo tal que $j^{-1}: Z_{\beta} \times \Lambda^{-\lambda} \longrightarrow X_{\beta+1} \times K_{\beta+1}$ é a seguinte função:

$$
j^{-1}\left(x_{\beta}, k_{\beta}, x_{\beta+1}, l\right)=\left(x_{\beta+1}, m\right)
$$

onde $m \in K_{\beta+1},\left.m\right|_{\omega_{\alpha_{\beta}}}=k_{\beta}$ e $\left.m\right|_{\lambda}=l$.

$j$ é uma função contínua pois $\chi, \pi_{\lambda}^{\beta+1}$ são funções contínuas e $j=\chi \triangle \pi_{\lambda}^{\beta+1}$.

Portanto a função $j$ é um homeomorfismo tal que $\chi=\pi_{1}^{\lambda} j$ onde $\pi_{1}^{\lambda}: Z_{\beta} \times \Lambda^{\lambda} \longrightarrow Z_{\beta}$ é a projeção na primeira coordenada.

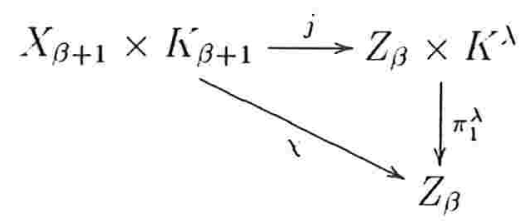

A ideia é construir o homeomorfismo $h_{\beta+1}$ a partir do homeomorfismo $j$, aplicando a hipótese de induçào ao espaço $Z_{\beta} \times K^{\lambda}$. Vemos que isto é possível pois por construçào $Z_{\beta}$ é um subespaço fechado de $X_{\beta}^{\prime} \times K_{\beta} \times X_{\beta+1}$ e

$$
X_{\beta} \times K_{\beta} \times X_{\beta+1} \text { é subespaço fechado de } K_{\beta} \times K_{\beta} \times K_{\beta+1} \approx K_{\beta+1}=K^{\omega_{\alpha_{3+1}}} \text {. }
$$

Como $|\lambda|=\left|\omega_{\alpha_{\beta+1}}-\omega_{\alpha_{\beta}}\right|=\omega_{\alpha_{\beta+1}}$, segue que $Z_{\beta}$ é um subespaço fechado de $\Lambda^{\prime}$. Por hipótese de indução, existe um homeomorfismo $g: Z_{\beta} \times K^{-\lambda} \longrightarrow Z_{\beta} \times K^{\lambda}$ tal que $\pi_{1}^{\lambda} g=\pi_{1}^{\lambda}$ e $f i x(g)=j\left(F_{\beta+1}\right)$.

Seja $f_{\beta+1}: X_{\beta+1} \times K_{\beta+1} \longrightarrow X_{\beta+1} \times K_{\beta+1}$ o homeomorfismo $f_{\beta+1}=j^{-1} g j$. Entào $f i x\left(f_{\beta+1}\right)=F_{\beta+1}$ e $\backslash f_{\beta+1}=\pi_{1}^{\curlywedge} j\left(j^{-1} g j\right)=\pi_{1}^{\curlywedge} g j=\pi_{1}^{\lambda} j=\chi$.

Seja também $g_{3}: Z_{\beta} \longrightarrow Z_{\beta}$ o homeomorfismo $g_{\beta}=h_{\beta} \varphi \triangle \psi$. Definimos entào

$$
h_{\beta+1}=f_{3+1} j^{-1}\left(g_{\beta} \times i d_{K^{\lambda}}\right) j
$$


$h_{\beta+1}$ assim definido é um homeomorfismo pois é composiçào de homeomorfismos. Vamos provar agora as propriedades $(i)_{\beta+1},(\text { ii })_{\beta+1},(\text { iii })_{\beta+1}$ :

$$
\begin{aligned}
\chi h_{\beta+1}\left(x_{3+1}, k_{\beta+1}\right) & =\chi f_{\beta+1} j^{-1}\left(g_{\beta} \times i d_{K^{-\lambda}}\right) j\left(x_{\beta+1}, k_{\beta+1}\right) \\
& =\chi j^{-1}\left(g_{\beta} \times i d_{K^{-\lambda}}\right) j\left(x_{\beta+1}, k_{\beta+1}\right) \\
& =\chi j^{-1}\left(g_{\beta} \times i d_{K^{-\lambda}}\right)\left(\left(\left.x_{\beta+1}\right|_{\omega_{\alpha_{\beta}}},\left.k_{\beta+1}\right|_{\omega_{\alpha_{\beta}}}, x_{\beta+1}\right),\left.k_{\beta+1}\right|_{\lambda}\right) \\
& =\chi j^{-1}\left(g_{\beta}\left(\left.x_{\beta+1}\right|_{\omega_{\alpha_{\beta}}},\left.k_{\beta+1}\right|_{\omega_{\alpha_{\beta}}}, x_{\beta+1}\right),\left.k_{\beta+1}\right|_{\lambda}\right) \\
& =\chi j^{-1}\left(h_{\beta}\left(\left.x_{\beta+1}\right|_{\omega_{\alpha_{\beta}}},\left.k_{\beta+1}\right|_{\omega_{\alpha_{\beta}}}\right), x_{\beta+1},\left.k_{\beta+1}\right|_{\lambda}\right) \\
& =\left(h_{\beta}\left(\left.x_{\beta+1}\right|_{\omega_{\alpha_{\beta}}},\left.k_{\beta+1}\right|_{\omega_{\alpha_{\beta}}}\right), x_{\beta+1}\right) \\
& =\left(h_{\beta}\left(p_{\beta}^{\beta+1} \times \pi_{\beta}^{\beta+1}\right) \triangle \pi_{1}^{\beta+1}\right)\left(x_{\beta+1}, k_{\beta+1}\right)
\end{aligned}
$$

Por outro lado temos que:

$$
\begin{aligned}
\backslash h_{, 3+1}\left(x_{3+1}, k_{\beta+1}\right) & =\left(\left(p_{\beta}^{\beta+1} \times \pi_{\beta}^{\beta+1}\right) \triangle \pi_{1}^{\beta+1}\right) h_{\beta+1}\left(x_{\beta+1}, k_{\beta+1}\right) \\
& =\left(\left(p_{\beta}^{\beta+1} \times \pi_{\beta}^{\beta+1}\right) h_{\beta+1} \triangle \pi_{1}^{\beta+1} h_{\beta+1}\right)\left(x_{\beta+1}, k_{\beta+1}\right)
\end{aligned}
$$

Portanto $\left(p_{3}^{3+1} \times \pi_{3}^{\beta+1}\right) h_{\beta+1}=h_{\beta}\left(p_{\beta}^{\beta+1} \times \pi_{\beta}^{\beta+1}\right)$ e $\pi_{1}^{\beta+1} h_{\beta+1}=\pi_{1}^{\beta+1}$. Temos então $\left(i_{\beta+1}\right.$ e $(\text { iii })_{3+1}$. Por definição de $h_{\beta+1}$, fix $\left(h_{\beta+1}\right)=\operatorname{fix}\left(f_{\beta+1}\right)=F_{\beta+1}$ e temos (ii) $\beta+1$.

- Se $\tau=u_{\alpha+1}$. para cada ponto $\left(x_{\beta}, k_{\beta}, x_{\beta+1}\right) \in Z_{\beta}$, temos que $\gamma^{-1}\left(x_{\beta}, k_{\beta} . x_{3=1}\right)=\left\{\left(x_{\beta+1}, \hat{k}_{\beta+1}\right) \in X_{\beta+1} \times K_{\beta+1}:\left.\hat{k}_{\beta+1}\right|_{\beta}=k_{\beta}\right\} \approx K^{\prime}$ onde $\lambda=\omega_{\alpha}$. Definimos a função $\hat{j}: X_{\beta+1} \times K_{\beta+1} \longrightarrow Z_{\beta} \times K^{\lambda}$ por:

$$
\hat{j}\left(x_{\beta+1}, k_{\beta+1}\right)=\left(\left(\left.x_{\beta+1}\right|_{\beta},\left.k_{\beta+1}\right|_{\beta}, x_{\beta+1}\right),\left.k_{\beta+1}\right|_{(\beta+1)-\beta}\right)
$$

$\hat{j}$ é um homeomorfismo tal que $\gamma=\pi_{i}^{\lambda} \hat{j}$ onde $\pi_{1}^{\lambda}: Z_{\beta} \times \kappa^{-\lambda} \longrightarrow Z_{\beta}$ é a projeçào na primeira coordenada.

Neste caso temos que o espaço $X_{\beta} \times K_{\beta} \times X_{\beta+1}$ é homeomorfo a $K^{\omega_{\alpha}}$, portanto $Z_{\beta}$ é um subespaço fechado de $K^{\omega_{\alpha}}$. Logo por hipótese de indução existe um homeomorfismo $\hat{g}: Z_{\beta} \times K^{\omega_{\alpha}} \longrightarrow Z_{\beta} \times K^{\omega_{\alpha}}$ tal que $\pi_{1}^{\beta+1} \hat{g}=\pi^{\beta+1}$ e $f i x(\hat{g})=\hat{j}\left(F_{\beta+1}\right)$. Seja $\hat{f}_{\beta+1}: X_{\beta+1} \times K_{\beta+1} \longrightarrow X_{\beta+1} \times K_{\beta+1}$ o homeomorfismo 
$\hat{f}_{\beta+1}=\hat{j}^{-1} \hat{g} \hat{j}$. Entào fix $\left(\hat{f}_{\beta+1}\right)=F_{\beta+1}$ e $\chi \hat{f}_{\beta+1}=\chi$. Se $g_{\beta}=h_{\beta} \varphi \Delta \psi$, definimos

$$
h_{\beta+1}=\hat{f}_{\beta+1} \hat{j}^{-1}\left(g_{\beta} \times i d_{K^{-\alpha \alpha} \alpha}\right) \hat{j}
$$

$h_{\beta+1}$ é homeomorfismo e verifica as propriedades $(i)_{\beta+1},(i i)_{\beta+1},(i i i)_{\beta+1}$. A prova é análoga ao caso $\tau$ singular.

Teorema 4.10 Sejam $\tau \geq \omega$ e $X$ um espaço compacto zero-dimensional de peso $\leq \tau$. Então a projeção $\pi_{1}: X \times\left(Z_{2}\right)^{\tau} \longrightarrow X$ é regular. Em particular, o produto $\mathrm{X} \times\left(Z_{2}\right)^{\top}$ tem $\mathrm{CIPH}$.

\section{Demonstração:}

Observamos que o espaço $X$ pode ser imerso em $\left(Z_{2}\right)^{\tau}$. Consequentemente, pelo teorema (4.9), basta considerar o caso $\tau=\omega$.

Seja $F$ um subconjunto fechado de $X \times\left(Z_{2}\right)^{\omega}$. Como $X \times\left(Z_{2}\right)^{\omega}$ é zero-dimensional com base enumerável, $X \times\left(Z_{2}\right)^{\omega}-F$ pode-se escrever como união enumerável disjunta $\bigcup_{i=1}^{\infty} C_{i}$ de subconjuntos abertos e fechados de $X \times\left(Z_{2}\right)^{\omega}$. A restrição $\left.\pi_{1}\right|_{C_{n}}: C_{n} \longrightarrow \pi_{1}\left(C_{n}\right), n \in \omega$ é uma d-aplicação tal que para cada $x \in \pi_{1}\left(C_{n}\right)$ temos

$$
\left(\pi_{1} \mid C_{n}\right)^{-1}(x)=\pi_{1}^{-1}(x) \cap C_{n}=\left(\{x\} \times\left(Z_{2}\right)^{\omega}\right) \cap C_{n}
$$

Portanto para cada $x \in \pi_{1}\left(C_{n}\right),\left(\left.\pi_{1}\right|_{C_{n}}\right)^{-1}(x)$ é homeomorfo a um subconjunto aberto e fechado de $\left(Z_{2}\right)^{\omega}$, logo $\left(\pi_{1} \mid C_{n}\right)^{-1}(x)$ é homeomorfo a $\left(Z_{2}\right)^{\omega}$ i.e. $\left(\pi_{1} \mid C_{n}\right)^{-1}(x)$ é um subconjunto completo de $\left(Z_{2}\right)^{\omega}$. Pelo lema (4.7), para cada $n \in \omega$ existe um homeomorfismo $t_{n}: \pi_{1}\left(C_{n}\right) \times\left(Z_{2}\right)^{\omega} \longrightarrow C_{n}$ tal que $\pi_{1} t_{n}=\pi_{C_{n}}$ onde $\pi_{C_{n}}: \pi_{1}\left(C_{n}\right) \times\left(Z_{2}\right)^{\omega} \longrightarrow \pi_{1}\left(C_{n}\right)$ denota a projeção na primeira coordenada.

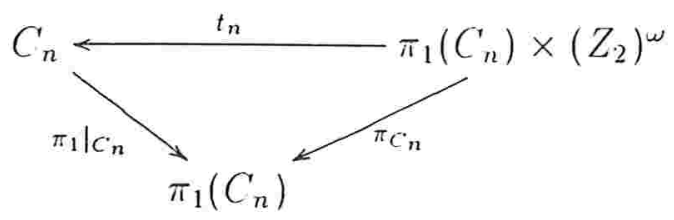


Seja $d$ uma métrica em $X \times\left(Z_{2}\right)^{\omega}$. Para cada $n \in \omega$ escolhemos um homeomorfismo $f_{n}:\left(Z_{2}\right)^{\omega} \longrightarrow\left(Z_{2}\right)^{\omega}$ sem pontos fixos e próximo o suficiente da função identidade. de modo que o homeomorfismo $g_{n}: C_{n} \longrightarrow C_{n}$ definido por $g_{n}=t_{n}\left(i d_{\pi_{1}\left(C_{n}\right)} \times f_{n}\right) t_{n}^{-1}$ seja tal que $d\left(g_{n}(x), x\right)<1 / n \quad \forall x \in C_{n}$. Observamos que para cada $n, g_{n}$ conserva a primeira coordenada, ou seja que $\pi_{1} g_{n}=\left.\pi_{1}\right|_{C_{n}}$.

Consideramos a função $h: X \times\left(Z_{2}\right)^{\omega} \longrightarrow X \times\left(Z_{2}\right)^{\omega}$ definida por :

$$
h(x)= \begin{cases}x & \text { se } x \in F, \\ g_{n}(x) & \text { se } x \in C_{n} .\end{cases}
$$

$h$ é uma função bijetora pois para cada $n, g_{n}$ é um homeomorfismo e $C_{i} \cap C_{j}=\emptyset$

se $i \neq j$.

$h$ é contínua:

Seja $x \in X \times\left(Z_{2}\right)^{\omega}$. Se $x \notin F$, então $x \in C_{k}$ para algum $k \in \omega$. Como $C_{k}$ é aberto e $\left.h\right|_{C_{k}}=g_{k}$ temos que $h$ é contínua em $x$.

Se $x \in F$, seja $B(x, \varepsilon)$ vizinhança aberta de $x$ e seja $A=\{n \in \omega: 1 / n \geq \varepsilon / 2\}$.

Como $A$ é um conjunto finito ou $A=\emptyset$, segue que $V=B(x, \varepsilon / 2)-\bigcup_{n \in A} C_{n}$ é um conjunto aberto. Para cada $y \in V$ temos que

$$
d(x, h(y)) \leq d(x, y)+d(y, h(y))<\varepsilon / 2+\varepsilon / 2
$$

Logo $h(y) \in B(x, \varepsilon) \forall y \in V$.

$h^{-1}$ é contínua:

Para cada $n \in \omega$ e para cada $x \in C_{n}$ temos:

$$
d\left(x, g_{n}^{-1}(x)\right)=d\left(g_{n}\left(g_{n}^{-1}(x)\right), g_{n}^{-1}(x)\right)<1 / n
$$

Logo a demonstraçào de que $h^{-1}$ é contínua é análoga a prova de $h$ contínua. $h$ preserva a primeira coordenada pois para cada $n, g_{n}$ preserva a primeira coordenada. Temos também que $f i x(h)=F$ pois para cada $n \in \omega g_{n}$ não tem pontos fixos.

Corolário 4.11 O cubo de Cantor $\left(Z_{2}\right)^{\tau}$ tem CIPH para todo cardinal infinito $\tau$. 


\section{APÊNDICE}

Definição A.1 Sejam $\kappa$ um cardinal infinito e $A \in[\kappa]^{\omega}$. Se $f: \prod_{\alpha<\kappa} X_{\alpha} \longrightarrow X$ é uma funçìo contínua, onde para cada $\alpha X_{\alpha}$ é um espaço topológico, dizemos que $f$ depende de $A$ se e somente se $\forall x, y \in \prod_{\alpha<r} X_{\alpha}\left(\pi_{A}(x)=\pi_{A}(y) \Rightarrow f(x)=f(y)\right)$.

Definição A.2 Sejam $f: \prod_{\alpha<\kappa} X_{\alpha} \longrightarrow \prod_{\alpha<\kappa} X_{\alpha}$ uma função contínua, $e$ $f_{A}: \prod_{\alpha<\kappa} X_{\alpha} \longrightarrow \prod_{\alpha \in A} X_{\alpha}$ a função $f_{A}=\pi_{A} \circ f$. Se $f_{A}$ depende de $A$, definimos a funçào $f^{A}: \prod_{\alpha \in A} X_{\alpha} \longrightarrow \prod_{\alpha \in A} X_{\alpha}$ como segue:

$$
f^{A}\left(\pi_{A}(x)\right)=\pi_{A}(f(x))
$$

Teorema A.3 (Stone-Weierstrass) Se um anel $P$ de funções contínuas a valores reais, definidas num espaço compacto $X$, contém todas as funções constantes, separa pontos e é fechado respeito a convergência uniforme, então $P$ coincide com o anel de todas as funçóes contínuas a valores reais definidas em X (ver [Engs9]).

Teorema A.4 Seja $f: \prod_{\alpha<\kappa} X_{\alpha} \longrightarrow X$ uma função contínua. Se X'é um espaço métrico separável e para cada $\alpha<\kappa X_{\alpha}$ é um espaço compacto, então $f$ depende de algum $A \in[\kappa]^{\omega}$.

\section{Demonstração:}

Primeiro consideramos $X=\mathbb{R}$ e aplicamos o teorema de Stone-Weierstrass.

Seja $P=\left\{f: \prod_{\alpha<\kappa} X_{\alpha}^{r} \longrightarrow \mathbb{R}: f\right.$ é contínua e depende de $\left.S_{f} \in[r]^{\omega}\right\}$ $(P,+,$.$) é um anel e P$ contém todas as funções constantes. Para ver que $P$ separa pontos, sejam $x, y \in \prod_{\alpha<\kappa} X_{\alpha}$. Se $x \neq y$, existe $\beta<\kappa$ tal que $\pi_{\{\beta\}}(x) \neq \pi_{\{\beta\}}(y)$. Seja $f: X_{\{\beta\}} \longrightarrow \mathbb{R}$ uma função contínua tal que $f\left(\pi_{\{\beta\}}(x)\right) \neq f\left(\pi_{\{\beta\}}(y)\right)$.

$$
\prod_{\alpha<\kappa} X_{\alpha} \stackrel{\pi_{\{\beta\}}}{\longrightarrow} X_{\{\beta\}} \stackrel{f}{\longrightarrow} \mathbb{R}
$$


Temos entào que $f \circ \pi_{\{\beta\}}(x) \neq f \circ \pi_{\{\beta\}}(y)$ e como $f \circ \pi_{\{\beta\}}$ é contínua e depende de $\{\beta\}$, pertence a $P$.

Falta provarmos que $P$ é fechado para a convergência uniforme. Para isto, consideramos uma sequência $\left(f_{n}\right)_{n \in \omega}$ em $P$, tal que converge uniformemente a uma função $f: \prod_{\alpha<\kappa} X_{\alpha} \longrightarrow \mathbb{R}$.

Como $f$ é contínua, só falta ver que depende de algum $S \in[\kappa]^{\omega}$.

$f_{n} \in P \forall n \in \omega$. então para cada $n$ existe $S_{n} \in[\kappa]^{\omega}$ tal que $f_{n}$ depende de $S_{n}$. Seja $S=\bigcup_{n \in \omega} S_{n} \quad$ e sejam $\quad x, y \in \prod_{\alpha<n} X_{\alpha} \operatorname{com} \quad \pi_{S}(x)=\pi_{S}(y)$.

Para $\varepsilon>0$ arbitrário, seja $n_{0} \in \omega$ tal que $\left|f_{n}(x)-f(x)\right|<\varepsilon / 2 \quad \forall n \geq n_{0}, \quad \forall x \in \prod_{\alpha<n} X_{\alpha} \quad$ e seja $m>n_{0}$. Temos que:

$$
|f(x)-f(y)| \leq\left|f(x)-f_{m}(x)\right|+\left|f_{m}(x)-f_{m}(y)\right|+\left|f_{m}(y)-f(y)\right|
$$

Como $f_{m}$ depende de $S, f_{m}(x)=f_{m}(y)$. Então $|f(x)-f(y)|<\varepsilon$, e como $\varepsilon$ é arbitrário temos que $f(x)=f(y)$. Portanto $f$ depende de $S$.

Entào pelo teorema de Stone-Weierstrass $P=\left\{f: \prod_{\alpha<k} X_{\alpha} \longrightarrow \mathbb{R}: f\right.$ é contínua $\}$ Seja agora $X^{\prime}$ um espaço métrico separável qualquer, e seja $i$ uma imersão de $X^{\prime}$ em $\mathbb{R}^{\omega}$.

Para cada $n \in \omega$, seja $g_{n}=\pi_{\{n\}} \circ i \circ f$.

$$
\prod_{\alpha<\kappa} X_{\alpha} \stackrel{f}{\longrightarrow} X \stackrel{i}{\hookrightarrow} \mathbb{R}^{\omega} \stackrel{\pi_{\{n\}}}{\longrightarrow} \mathbb{R}
$$

Como $g_{n}$ é uma função a valores reales, pelo mostrado acima tamos que $g_{n}$ depende de $A_{n} \in[k]^{\omega}$.

Seja $A=\bigcup_{n \in \omega} A_{n}$, vamos provar que $f$ depende de $A$. Se $x, y \in \prod_{\alpha<r} X_{\alpha}$ são tais que $\pi_{A}(x)=\pi_{A}(y)$, suponhamos que $f(x) \neq f(y)$. Como $i$ é injetora, $i(f(x)) \neq i(f(y))$, logo existe $n_{0} \in \omega$ tal que $\pi_{\left\{n_{0}\right\}}(i f(x)) \neq \pi_{\left\{n_{0}\right\}}(i f(y))$ i.e. $g_{n_{0}}(x) \neq g_{n_{0}}(y)$.

Mas $A_{n_{0}} \subset A$, entào $\pi_{A_{n_{0}}}(x)=\pi_{A_{n_{0}}}(y)$ e como $g_{n_{0}}$ depende de $A_{n_{0}}$ temos que $g_{n_{0}}(x)=g_{n_{0}}(y)$. Esta contradição mostra que $f$ depende de $A$. 
Lema A.5 Suponhamos que para cada $\alpha<\kappa, X_{\alpha}$ é um espaço métrico compacto. Seja $f: \prod_{\alpha<\kappa} X_{\alpha} \longrightarrow \prod_{\alpha<\kappa} X_{\alpha}$ uma função contínua. Então existe um conjunto c.u.b $C_{f} \subseteq[\kappa]^{\omega}$ tal que para cada $A \in C_{f}, f_{A}$ depende de $A$.

\section{Demonstração:}

Para cada $\alpha \in \kappa$, seja $f_{\{\alpha\}}: \prod_{\alpha<\kappa} X_{\alpha} \longrightarrow X_{\alpha}, f_{\{\alpha\}}=\pi_{\{\alpha\}} \circ f$.

Por teorema (A.4) temos que para cada $\alpha \in \kappa$, existe $A_{\alpha} \in[\kappa]^{\omega}$ tal que $f_{\{\alpha\}}$ depende de $A_{\alpha}$.

Seja $C_{f}=\left\{X \in[\kappa]^{\omega}: \forall \alpha \in X, A_{\alpha} \subseteq X^{r}\right\}$

* $C_{f}$ é fechado:

Seja $\left(X_{n}\right)_{n \in \omega} \subseteq C_{f}$ uma sequência crescente. Se $\alpha \in \bigcup_{n \in \omega} X_{\alpha}$, então existe $n_{0} \in \omega$ tal que $\alpha \in X_{n_{0}}$, e como $X_{n_{0}} \in C_{f}$ temos que $A_{\alpha} \subseteq X_{n_{0}}$, portanto $A_{\alpha} \subseteq \bigcup_{n \in \omega} X_{\alpha}$.

Temos então:

$$
\alpha \in \bigcup_{n \in \omega} X_{n} \Rightarrow A_{\alpha} \subseteq \bigcup_{n \in \omega} X_{n} \Rightarrow \bigcup_{n \in \omega} X_{n} \in C_{f}
$$

* $C_{f}$ é não limitado em $[\kappa]^{\omega}$

Sejam $X \in[\kappa]^{\omega}$ e $Y_{1}=X \bigcup\left(\bigcup_{\alpha \in X} A_{\alpha}\right)$.

$Y_{1} \in[\kappa]^{\omega}$ pois é reunião enumerável de subconjuntos enumeráveis de $\kappa$.

Para cada $n>1$, definimos $Y_{n+1}=Y_{n} \bigcup\left(\bigcup_{\alpha \in Y_{n}} A_{\alpha}\right)$. Temos que $Y_{n+1} \in[\kappa]^{\omega} \forall n \in \omega$.

Seja $Y=\bigcup_{n \in \omega} Y_{n}$, então $Y \in C_{f}$ e $X \subseteq Y$.

Falta provar que $f_{A}$ depende de $A$ para todo $A \in C_{f}$.

Se $A \in C_{f}, A_{\alpha} \subseteq A \quad \forall \alpha \in A$. Sejam $x, y \in \prod_{\alpha<\kappa} X_{\alpha}$ tais que

$\pi_{A_{\alpha}}(x)=\pi_{A_{\alpha}}(y) \quad \forall \alpha \in A$. Então $f_{\{\alpha\}}(x)=f_{\{\alpha\}}(y) \forall \alpha \in A$. Logo

$\prod_{\alpha \in A} f_{\{\alpha\}}(x)=\prod_{\alpha \in A} f_{\{\alpha\}}(y)$, ou seja $f_{A}(x)=f_{A}(y)$.

Definição A.6 Dizemos que um espaço topológico $X$ tem a propriedade do ponto fixo se e somente se para cada função contínua $f: X \longrightarrow X$ existe $x \in X$ tal que $f(x)=x$. 
Teorema A.7 (de Schauder) Seja E um espaço normado, $K \subset E$ compacto convexo. Então $l i$ tem a propriedade do ponto fixo. (ver [Hönīi]

Teorema A.8 Seja $\kappa$ um cardinal infinito. Suponhamos que para cada $\alpha<\kappa, X_{\alpha}$ é um subespaço compacto convexo de um espaço linear normado. Então $\prod_{\alpha<\kappa} X_{\alpha}$ tem a propriedade do ponto fixo.

\section{Demonstração:}

Para todo $F \in[\kappa]^{<\omega}, \prod_{\alpha \in F} X_{\alpha}$ é um espaço compacto convexo, então por teorema de Schauder, $\prod_{\alpha \in F} X_{\alpha}$ tem a propriedade do ponto fixo.

Seja $f: \prod_{\alpha<\kappa} X_{\alpha} \longrightarrow \prod_{\alpha<\kappa} X_{\alpha}$ uma função contínua.

Para cada $F \in[\kappa]^{<\omega}$ seja $Y_{F}=\left\{x \in \prod_{\alpha<\kappa} X_{\alpha}: \pi_{F}(f(x))=\pi_{F}(x)\right\}$

Suponhamos que $\bigcap_{F \in[\kappa]<\omega} Y_{F} \neq \emptyset$, e seja $x \in \bigcap_{F \in[\kappa]<\omega} Y_{F}$.

$$
x \in \bigcap_{F \in[\kappa]<\omega} Y_{F} \Rightarrow x \in Y_{\{\alpha\}} \forall \alpha<\kappa \Rightarrow \pi_{\{\alpha\}}(f(x))=\pi_{\{\alpha\}}(x) \forall \alpha<\kappa \Rightarrow f(x)=x
$$

Portanto basta provar que $\bigcap_{F \in[\kappa]<\omega} Y_{F} \neq \emptyset$. Para isto demonstraremos que :

(1) $Y_{F} \neq \emptyset \quad \forall F \in[r]^{<\omega}$

(2) $\left\{Y_{F}: F \in[\kappa]^{<\omega}\right\}$ tem a propriedade da interseção finita

(3) $Y_{F}$ é fechado $\forall F \in[k]^{<\omega}$

Como $\prod_{\alpha<\kappa} X_{\alpha}$ é compacto, as propriedades (1),(2) e (3) implicam que $\bigcap_{F \in[\kappa]<\omega} Y_{F} \neq \emptyset$.

(1) $Y_{F} \neq \emptyset \quad \forall F \in[\kappa]^{<\omega}$ :

Seja $x \in \prod_{\alpha<\kappa} X_{\alpha}, x=\pi_{F}(x) \times \pi_{\kappa-F}(x)$. Consideramos as seguintes funçòes :

* a projeção $\hat{\pi}_{F}: \prod_{\alpha \in F} X_{\alpha} \times\left\{\pi_{\kappa-F}(x)\right\} \longrightarrow \prod_{\alpha \in F}$ que é um homeomorfismo. e 
* a inclusào $i: \prod_{\alpha \in F} X_{\alpha} \times\left\{\pi_{\kappa-F}(x)\right\}$ que é uma imersão, e portanto contínua.

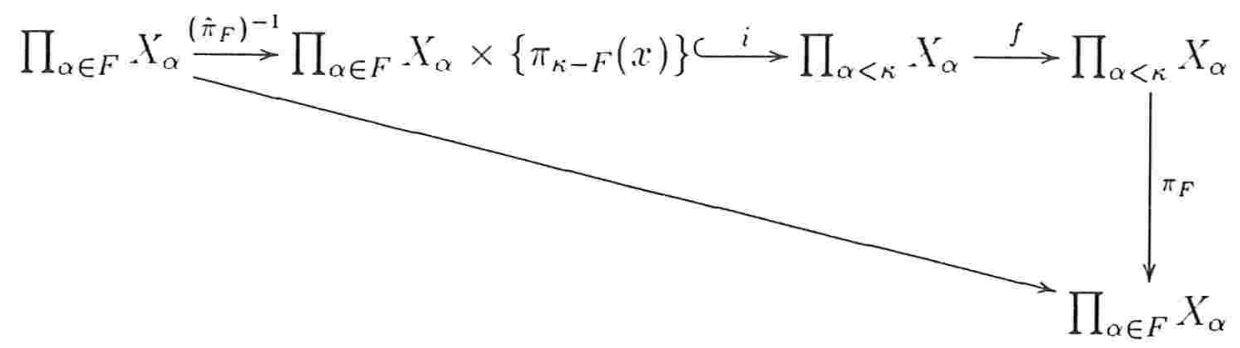

A função $\pi_{F} \circ f \circ i \circ \pi_{F}^{-1}: \prod_{\alpha \in F} X_{\alpha} \longrightarrow \prod_{\alpha \in F} X_{\alpha}$ é entào contínua, e por teorema de Schauder tem ponto fixo. Seja $y_{F} \in \prod_{\alpha \in F} X_{\alpha}$ ponto fixo de $\pi_{F} \circ f \circ i \circ \pi_{F}^{-1}$, e seja $y=y_{F} \times\left\{\pi_{n-F}(x)\right\}$. Temos que $y_{F}=\pi_{F} \circ f \circ i \circ \pi_{F}^{-1}\left(y_{F}\right)=\pi_{F} \circ f \circ i(y)=\pi_{F} \circ f(y)$. Portanto $y \in Y_{F}$.

(2) $\left\{Y_{F}: F \in[\kappa]^{<\omega}\right\}$ tem a p.i.f:

Dados $F_{1}, \ldots, F_{m} \in F \in[\kappa]^{<\omega}, \operatorname{sejam} F=\bigcup_{i=1}^{m} F_{i} \quad$ e $\quad x \in Y_{F}$.

$x \in Y_{F} \Rightarrow \pi_{F}(f(x))=\pi_{F}(x) \Rightarrow \pi_{F_{i}}(f(x))=\pi_{F_{i}}(x) \Rightarrow x \in Y_{F}$ para $i=1, \ldots, m$ ou seja, $x \in \bigcap_{i=1}^{m} Y_{F_{i}}$.

(3) $Y_{F}$ é fechado $\forall F \in[\kappa]^{<\omega}$

Sejam $F \in[\kappa]^{<\omega}$ e $x \in \prod_{\alpha<\kappa} X_{\alpha}-Y_{F}$.

$x \notin Y_{F} \Rightarrow \pi_{F}(f(x)) \neq \pi_{F}(x) \Rightarrow \exists \beta \in F$ tal que $\pi_{\{\beta\}}(x) \neq \pi_{\{\beta\}}(f(x))$. Como $X_{\beta}$ é um espaço $T_{2}$, existem $U_{\beta}$ vizinhança aberta de $\pi_{\{\beta\}}(x)$ e $V_{\beta}$ vizinhança aberta de $\pi_{\{\beta\}}(f(x))$ tais que $U_{\beta} \cap V_{\beta}=\emptyset$

Sejam $U=U_{\beta} \times \prod_{\substack{\alpha \neq \alpha \\ \alpha \neq \beta}} X_{\alpha}$ e $V=V_{\beta} \times \prod_{\substack{\alpha \neq \alpha \\ \alpha \neq \beta}}^{\substack{\alpha<\alpha \\ \alpha}}$ vizinhança aberta de $x$ e $f(x)$ respectivamente.

Como $f$ é contínua, existe $W$ vizinhança aberta de $x, W \subset U$ tal que $f(y) \in V \forall y \in W$. Então temos que :

$$
\forall y \in W:\left\{\begin{array}{l}
\pi_{\{\beta\}}(y) \in U_{\beta} \quad \mathrm{e} \\
\pi_{\{\beta\}}(f(y)) \in V_{\beta}
\end{array}\right.
$$

e como $\quad U_{\beta} \cap V_{\beta}=\emptyset$, temos $\pi_{\{\beta\}}(y) \neq \pi_{\{\beta\}}(f(y))$. 
Portanto $\pi_{F}(y) \neq \pi_{F}(f(y)) \quad \forall y \in W$.

Logo $\prod_{\alpha<\kappa} X_{\alpha}^{\prime}-Y_{F}$ é aberto. 


\section{Referências Bibliográficas}

[CM9Ti] Alex ('higogidze and John R. Martin. Fixed point sets of autohomeomorphisms of uncountable products. Topology Appl.80 pp 63- 1 1. 1997.

[Eng89] Ryszard Engelking. General Topology. Sigma series in pure Mathematics; Vol.6. Helderman Verlag, 1989.

[Hod84] R. Hodel. Cardinal Functions I. Handbook of set-theoretic topology. cap 1.

[Hönīi] Chaim Samuel Hönig. Aplicaçoes da Topologia à Análise. Instituto de Matemática Pura e Aplicada. CNPq. 1.97Ti. (Projeto Euclides).

[Kos92] Piotr Kioszmider. On the Complete invariance property in some uncountable products. Can.Math.Bull. 35 pp 221-229, 1992.

[Kun80] Kenneth Liunen. Set Theory: Un introduction to independence proofs. Studies in Logic and the foundations of Mathematics: Vol.102. North-Holland. 1980.

[KV84] Kenneth Kunen and Jerry E. Vaughan. Handbook of set-theoretic topology: lorth-Holland. 1984.

[Limiī] Elon Lages Lima. Espaços métricos. Instituto de Matemática Pura e Aplicada. CXPq. 197T. (Projeto Euclides). 
[Mars\&] John R. Martin. Fixed point sets of homeomorphisms of metric products. Proc. Amer. Math. Soc. 103 pp 1293-1298. 1988.

[Mils8] J.van Mill. Infinite Dimensional Topology: Prerequisites and Introduction. North-Holland . Mathematical Library Vol.43. North-Holland, 1988.

[MWS4] John R. Martin and William Weiss. Fixed point sets of Metric and Vonmetric Spaces. Trans.Amer.Math.Soc. 284 pp.33i-3.53, 1984.

[Rob6i] Herbert Robbins. Some complements to Brower's fixed point theorem. Israel .J. Math. 5 pp225-226. 1967.

[Rud:56] Walter Rudin. Homogeneity problems in the theory of C'ech compactifications. Duke Math J. 23 pp 409-419. 1956.

[Sch69] H. Shirmer. On fixed point sets of the n-ball. Israel J. Math. 10 pp.46-50, 1969

[Sch81] H.Shimer. Fixed point sets of continuous selfmaps, in:Fixed Point Theory. ('onf. Proc.. Sherbrooke,1980. Lecture Notes in Math. 866 pp.417-428. 1981.

[Sir68] S. Sirota. The spectral representation of spaces of closed subsets of bicompacta. Soviet.Math.Dokl. 9 pp.997-1000, 1963.

[T.J97] Artur Tomita e Lucia Junqueira. Topologia Cieral- $1^{\circ}$ semestre 1997. Notas de aula do IME/LSP, 1997.

[War73] L.E. Ward J. Fixed point sets. Pacific J. Math. 47 pp.55:3-565, 1973. 